\title{
Shear flow experiments: Characterizing the onset of turbulence as a phase transition
}

\author{
Dissertation \\ for the award of the degree \\ "Doctor rerum naturalium" \\ of the Georg-August-Universität Göttingen \\ within the doctoral program GGNB \\ of the Georg-August University School of Science (GAUSS)
}

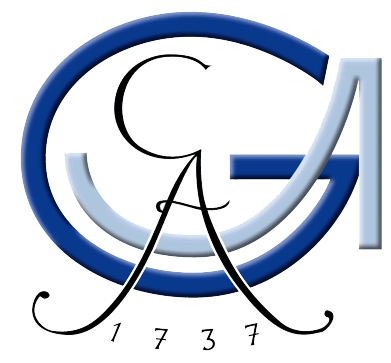

submitted by

Kerstin Avila

from Karlsruhe

Göttingen 2013 


\section{Thesis Committee}

\section{Prof. Dr. Björn Hof}

Nonlinear Dynamics and Turbulence, Institute of Science and Technology Austria

\section{Prof. Dr. Eberhard Bodenschatz}

Fluid Dynamics, Pattern Formation, and Biocomplexity,

Max Planck Institute for Dynamics and Self-Organization

Prof. Dr. Andreas Tilgner

Institute of Geophysics, University of Göttingen

\section{Members of the Examination Board}

\section{Referee: Prof. Dr. Björn Hof}

Nonlinear Dynamics and Turbulence, Institute of Science and Technology Austria

$2^{\text {nd }}$ Referee: Prof. Dr. Eberhard Bodenschatz

Fluid Dynamics, Pattern Formation, and Biocomplexity,

Max Planck Institute for Dynamics and Self-Organization

\section{Further members of the Examination Board}

\section{Prof. Dr. Andreas Tilgner}

Institute of Geophysics, University of Göttingen

Prof. Dr. Andreas Dillman

Institute of Aerodynamics and Flow Technology, German Aerospace Center

Prof. Dr. Jürgen Vollmer

Dynamics of Complex Fluids, Max Planck Institute for Dynamics and Self-Organization

\section{Dr. Jean-Christophe Baret}

Droplets, Membranes and Interfaces, Max Planck Institute for Dynamics and Self-Organization

Date of oral examination: 05.11.2013 


\section{Contents}

$\begin{array}{ll}\text { Abstract } & 5\end{array}$

$\begin{array}{ll}\text { Motivation } & 7\end{array}$

1 Introduction 9

1.1 Transition to turbulence in the 19 th century . . . . . . . . . . . 9

1.1.1 Navier-Stokes equation, Hagen-Poiseuille . . . . . . . . . . . . 9

1.1.2 Reynolds pioneering experiments . . . . . . . . . . . . 11

1.2 Transition to turbulence in the 20 th century . . . . . . . . . . . . . . 14

1.2.1 Rotta's pipe flow experiments . . . . . . . . . . . . . . . 14

1.2.2 The invention of the Taylor-Couette system and its pioneering studies ........................ 17

1.2.3 Linear instabilities: supercritical transition . . . . . . . . . . 19

1.2.4 Linearly stable basic flow: subcritical transition . . . . . . . . . 21

1.2.5 Statistical approach: Directed percolation and Coupled map lattices 23

1.2.6 Statistical analysis of flow dynamics . . . . . . . . . . . . 27

1.3 Transition to turbulence in the 21 st century . . . . . . . . . . . . 29

1.3.1 Transient turbulence in pipe flow . . . . . . . . . . . . . . 29

1.3.2 Spatial proliferation of turbulence in pipe flow . . . . . . . 31

1.3.3 Plane Couette flow: 1st or 2nd order phase transition? . . . . . . 32

1.3.4 Taylor-Couette flow: Analogy and differences to plane Couette flow . . . . . . . . . . . . . . . 34

2 The onset of turbulence in pipe flow 37

2.1 Abstract . . . . . . . . . . . . . . . . . . . 37

2.2 Introduction . . . . . . . . . . . . . . . . . . . . . . . . . . . . . . 39

2.3 Long-pipe experiments . . . . . . . . . . . . . . . . . . 39

2.4 Simulations . . . . . . . . . . . . . . . . . 42

2.5 Puff splitting . . . . . . . . . . . . . . . . . 42

2.6 Characteristic time of memoryless process . . . . . . . . . . . . . 42

2.7 Critical point . . . . . . . . . . . . . . . . 45

2.8 Conclusion . . . . . . . . . . . . . . . . . . . 47

2.9 Acknowledgments . . . . . . . . . . . . . . . . . . 47

2.10 Supplementary Online Material . . . . . . . . . . . . . . . . . . . . . . 48

2.10.1 Materials and Methods . . . . . . . . . . . . . . . . . . . . . 48

2.10 .2 Supporting Figures . . . . . . . . . . . . . . . . . . 51 
2.10 .3 Supporting Table . . . . . . . . . . . . . . . . . . 54

3 High-precision Taylor-Couette experiment $\quad 57$

3.1 Abstract . . . . . . . . . . . . . . . . 57

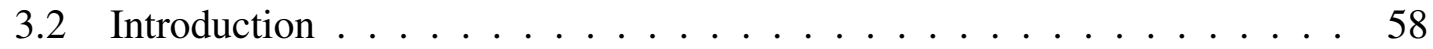

3.2.1 Applications of the Taylor-Couette setup . . . . . . . . . . 58

3.2.2 High precision Taylor-Couette apparatus . . . . . . . . . . . 60

3.3 System Description . . . . . . . . . . . . . . . . . . 63

3.3 .1 General setup . . . . . . . . . . . . . . . . 63

3.3.2 Geometry and materials of the cylinders and the endplates . . . . 64

3.3.3 Bearing, sealing and balancing . . . . . . . . . . . . 66

3.3.3.1 Bottom bearing unit . . . . . . . . . . . 67

3.3.3.2 Top bearing units . . . . . . . . . . . . . 69

3.3 .4 Aspect ratio control . . . . . . . . . . . . . . . 70

3.3.5 Rotation rate control . . . . . . . . . . . . . . . 72

3.3.6 Temperature control . . . . . . . . . . . . . . 75

3.3.7 Other (not yet implemented) possibilities . . . . . . . . . 76

3.4 Flow visualization . . . . . . . . . . . . . . . . . . . 77

3.4.1 Working fluid and tracers . . . . . . . . . . . . . 77

3.4 .2 Spatio-temporal analysis . . . . . . . . . . . . . 77

3.5 Examples of results . . . . . . . . . . . . . . . . . . 80

3.5.1 Onset of Taylor vortices for stationary outer cylinder $(\eta=0.98) \quad$. 80

3.5.2 Onset of the linear instability for counter-rotating cylinders with $\eta=0.98 \ldots \ldots \ldots \ldots \ldots \ldots$. . . . . . . . . . . . . . .

3.6 Discussion . . . . . . . . . . . . . . . . . 82

3.7 Acknowledgments . . . . . . . . . . . . . . 83

4 Phase transition in Couette flows $\quad 85$

4.1 Influence of the system size . . . . . . . . . . . . . . . 85

4.2 Influence of the accuracy of the experimental setup . . . . . . . . . 87

4.3 Phase transition in a large system with high accuracy . . . . . . . . . 88

5 Discussion $\quad 91$

5.1 Open question: Localization of turbulence . . . . . . . . . . . . . . . 95

$\begin{array}{lr}\text { Perspective } & 97\end{array}$

$\begin{array}{lr}\text { Bibliography } & 99\end{array}$

$\begin{array}{ll}\text { Acknowledgements } & 109\end{array}$

$\begin{array}{ll}\text { A Von Wirbelballen zur Turbulenz } & 111\end{array}$ 


\section{Abstract}

In this cumulative thesis the onset of turbulence in shear flows with a linearly stable laminar flow is investigated experimentally. The experiments are performed in a pipe and a Taylor-Couette setup, both with large system sizes. A high-precision Taylor-Couette experiment has been designed and three important main results have been obtained in the course of this study.

The first main result gives the solution to a question from the 19th century by one of the pioneers of fluid dynamics, Osborne Reynolds. He asked at what critical Reynolds number $R e_{c}$ pipe flow turns persistently turbulent. My experiments show it is at $R e_{c}=$ $2040 \pm 10$. The pressure driven pipe flow experiment had a length of more than 3300 pipe diameters and water was used as working fluid. Turbulence was induced in a controlled manner and its development detected downstream with pressure sensors. Large statistical ensembles were performed.

The second main result is to identify the mechanism that determine this transition between laminar flow and sustained turbulence. It is shown that purely temporal aspects like the Ruelle-Takens scenario or the statistical decay of turbulent patches are not sufficient to characterize it. Instead the spatial proliferation of turbulence, which is also a statistical process, has to be taken into account. The competition of the decay and the spreading of turbulence define $R e_{c}$. The larger picture emerging from these observations is that the onset of turbulence in pipe flow can be characterized as a non-equilibrium phase transition.

The third main result is that the phase transition is of second order. In contrast to the previous observation, this result was obtained in a flow between two concentric counterrotating cylinders (Taylor-Couette flow). In the selected parameter regime the dynamics resembles that of pipe flow, but with the advantage that the time scales are much shorter. Therefore it was possible to investigate the phase transition in more detail by analyzing the scaling of the mean turbulent fraction depending on Re. A system size 12 times larger than previous Couette experiments combined with a high accuracy and long observation times allowed it to measure substantially closer to the critical point than previously. The continuity of the transition could be identified for the first time, thereby contradicting interpretations from experiments in literature, but supported by models of pipe flow and recent numerical simulations of Couette flow.

A radius ratio of $\eta=0.98$ and an aspect ratio of about 260 were used and the working fluid, silicone oil, was seeded with Al-tracers for visualization. The flow was monitored with a high speed camera from which the mean turbulent fraction was obtained by image processing.

Another accomplishment of this thesis is the construction of the Taylor-Couette experiment that was used for the aforementioned investigation. Besides its large system size 
and high accuracy this setup offers a wide regime of parameters. The radius ratio can be easily changed from extremes of a thin inner cylinder (radius ratio $\eta=0.03$ ) to almost identical radii $(\eta=0.98)$, the aspect ratio can be dynamically varied during measurements and the rotation rates of the cylinders allow studies in the transitional as well as in the turbulent regime. By independently rotating the top and bottom lid the boundary condition can be adjusted to minimize endwall effects. The sophisticated bearing system is combined with several cooling circuits to provide a high precision during long-time measurements. Excellent optical access and index matching allow for optical measurement techniques. 


\section{Motivation}

Some seemingly simple questions turn out to be extremely tricky to answer, and few of them in addition turn out to reveal deep physical insights. The question addressed in the first part of this thesis is such a question - and indeed it was asked exactly 130 years ago by Osborne Reynolds, one of the pioneers in fluid dynamics. He asked himself under what conditions turbulence becomes sustained in a pipe. Or more precisely in his own words:

it became clear to me that if in a tube of sufficient length the water were at first admitted in a high state of disturbance, then as the water proceeded along the tube the disturbance would settle down into a steady condition, which condition would be one of eddies or steady motion, according to whether the velocity was above or below what may be called the real critical value' (Reynolds 1883).

In this thesis the 'real critical value'(Reynolds 1883) is determined. It is appealing that it could not be determined just because of an advancement of technical procedures or methods. It is a consequence of the knowledge that has been contributed by many researchers over the last century, and especially the last decade. In principle Reynolds could have answered his question - even though he would have had to spend many month in the lab. I am convinced he would have done it.

Luckily today we have measurement techniques that can be automated, so that another more general question could be attacked in the second part of this thesis that arose from the answer to Reynolds' question. The onset of turbulence in pipe flow can be characterized as a non-equilibrium phase transition (Chapter 2, Avila et al. (2011)). Models in pipe flow predict it to be of second order (Barkley 2011) and ongoing experiments ${ }^{1}$ are supporting this. But is this generally valid for linearly stable shear flows? Is the onset of turbulence in these flows a non-equilibrium phase transition of second order? Previous investigations in other shear flows (plane and Taylor-Couette flow) do not support this appealing idea and seem to undergo a first order transition (Bottin et al. 1998, Duguet et al. 2010). However, here the challenge is not only in the understanding of the ongoing physics, but in building an experiment, in which this can be accurately measured. Researchers have tried to do so for more than a decade (Prigent et al. 2003), but could not achieve it. In the second part of my thesis I designed, build and tested a complex highprecision (Taylor-Couette) experiment with which it could be shown that the transition is of second order. Furthermore, ongoing measurements will likely clarify if this transition belongs to the universality class of directed percolation. Although directed percolation

\footnotetext{
${ }^{1}$ private communication with Mukund Vasudevan
} 
serves as a model for many processes in nature (from the spreading of infections to forest fires), it has so far only been accurately measured in a single experiment of liquid crystals (Takeuchi et al. 2007).

My thesis is structured as follows: In the Introduction (Chapter 1), I draw the path that research has taken from Reynolds' pioneering pipe flow experiments to current research in transition to turbulence. The main focus is on canonical flows like pipe, plane Couette (flow between two shearing plates) and Taylor-Couette flows (flow between two concentric rotating cylinders) to concentrate on the underlying physical mechanisms. Chapter 2 consists of a publication published in Science, where the critical Reynolds number for the onset of turbulence in pipe flow is determined and the transition placed in the group of phase transitions (Avila et al. 2011). In Chapter 3 a technical description of the Taylor-Couette experiment is presented. This appeared in the Review of Scientific Instruments (Avila \& Hof 2013). Besides the technical description, the method of analysis is explained and it is shown that finite amplitude perturbations are avoided in the relevant regime. In Chapter 4 unpublished measurements on the phase transition in Taylor-Couette flows are presented. It is revealed that the phase transition is of second order in a system of sufficient size and accuracy and not of first order as reported in literature. In Chapter 5 the results are discussed altogether and put in a larger context, including a specific outlook on localized turbulence. The last part is a Perspective on the transition to turbulence in general. 


\section{Introduction}

The understanding and control of fluid dynamics accompanied civilization from its very beginnings and still poses one of the greatest challenges nowadays. By using the wind with sailing boats, humans were able to cross oceans and discover new continents. By building aqueducts the Romans made the first large step towards metropolitan cities, thereby overcoming also the main reason for the spreading of diseases. Later on, with a much deeper understanding of the underlying physical mechanism, one of the oldest dreams of mankind came true - flying.

In recent years applications of fluid dynamics range from Lab-on-a-chip devices on the nanometer scale up to predictions of the global climate change. These suggests that all basic questions have already been answered. But a closer look reveals that basic questions like: 'How does turbulence develop? What is the critical transition point?' are still unanswered. One of the largest problems in micro- and nanofluidics is for example to generate chaotic behavior, so that fluids become well mixed. In climate models it is crucial to understand the dynamics beyond statistical averages to determine critical transition points. If such a critical point is crossed once, the behavior changes qualitatively. For example, if the Earth's temperature rises by some degrees, than the Golf stream may stop and the climate in Europe may change drastically.

\subsection{Transition to turbulence in the 19th century}

\subsubsection{Navier-Stokes equation, Hagen-Poiseuille}

The first person asking these basic questions was Osborne Reynolds in the 19th century. What was known at that time is the Navier-Stokes equation, which correctly describes the motion of fluids. Prior to it, Euler (1752) first applied Newton's second law to fluids and derived an equation describing the motion of frictionless (inviscid) fluids. These equations are still widely used in aerodynamics and astrophysics. However, to describe flows close to walls and the onset of turbulence Euler's equations are not satisfactory. In such cases the internal friction of the fluid, characterized by its viscosity is of tremendous importance. Navier (1823) and Stokes (1845) combined in their equation for the first time the viscous force of the internal motion of the fluid with the Euler equation. As a boundary condition they assumed that the fluid has zero velocity where it is in contact with a stationary wall ('no-slip boundary condition'). Although the final form of the equation and boundary conditions were correctly derived by Stokes in 1845, it took much longer to verify its validity experimentally (see Section 1.2.2). 


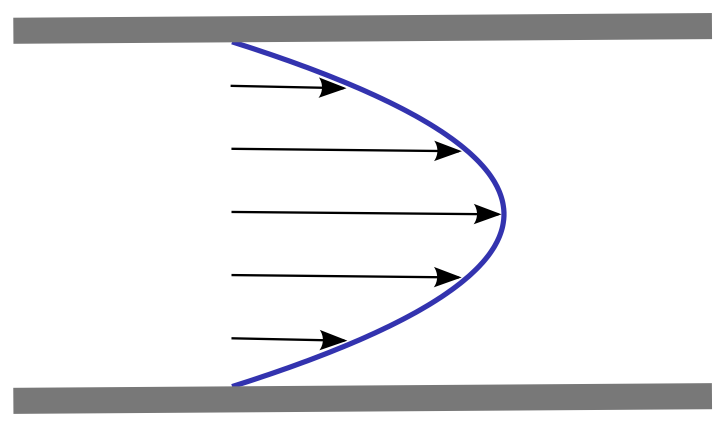

Figure 1.1: Hagen (1839) and Poiseuille (1840) analytically obtained the velocity profile of laminar pipe flow, which is parabolic.

The Navier-Stokes equation reads

$$
\rho\left(\frac{\partial \vec{u}}{\partial t}+(\vec{u} \cdot \nabla) \vec{u}\right)=-\nabla p+\mu \nabla^{2} \vec{u}+\vec{F}, \quad \nabla \cdot \vec{u}=0,
$$

where the first equation expresses the balance of linear momentum and the second conservation of mass. Here $\vec{u}$ is the velocity field, $p$ the pressure, $\rho$ and $\mu$ the fluid density and dynamic viscosity, and $\vec{F}$ an external force.

The Navier-Stokes equation is a nonlinear partial differential equation, which makes it very difficult to solve it. Mathematically it is not even clear, if a three dimensional solution always exists and if so, if it contains no singularities. A price of US\$1.000.000 is set by the Clay Mathematics Institute to solve this problem, which is one out of seven millennium prize problems (Carlson et al. 2006).

Only very few exact solutions of the Navier-Stokes equation are known. The perhaps most relevant one describes the parabolic velocity profile of a flow through a straight, circular pipe, as shown in Fig. 1.1. The flow assumes such a profile only after a certain distance from the pipe entrance, which is inversely proportional to the viscosity (Tietjens 1970). When the fluid enters the pipe from e.g. a large container, the streamwise velocity is nearly uniform across the bulk of the pipe. However, the no-slip boundary condition enforces zero-velocity at the pipe wall, which leads to a spatially developing boundary layer. While the fluid is flowing downstream, the boundary layer thickness increases due to viscosity. Once the effect of the viscosity has reached the centerline of the pipe, the velocity profile becomes parabolic. The corresponding volume flux $Q$ is proportional to the applied pressure difference and was obtained by Hagen (1839) and Poiseuille (1840):

$$
\text { Volume flux } Q=\frac{\left(p_{0}-p_{1}\right) R^{4}}{8 \mu l}
$$

with $\left(p_{0}-p_{1}\right)$ being the pressure drop between two positions at distance $l$ along the pipe, $R$ the radius of the pipe and $\mu$ the dynamic viscosity of the fluid. However, this linear relation is only valid for laminar flows as in the capillary pipes of Poiseuille. In larger pipes Hagen $(1854)^{1}$ and also Darcy $(1857)^{2}$ observed the onset of disordered motion (e.g. turbulence)

\footnotetext{
${ }^{1}$ mentioned inTietjens (1970)

${ }^{2}$ mentioned in Mullin (2011)
} 
when the flow velocity was increased. In this case, the Hagen-Poiseuille law was not valid any more. This is to my knowledge the first time that the dynamics of flows was divided in two different classes by flow visualization. The first one is the laminar flow, here the fluid is moving in nearby layers, no mixing between these layers is observed and the flow velocity is often constant with time. The other type is turbulent flow, here many vortices appear with different sizes, interacting in an unpredictable way, thereby mixing the fluid. In turbulent flows the friction of the flow is substantially increased, which is exactly what Hagen and Darcy observed in their pipes.

\subsubsection{Reynolds pioneering experiments}

But why the linear dependence of the friction with the fluid velocity is not valid any more when turbulence sets in was not clear at all.

'This accidental fitness of the theory to explain certain phenomena while entirely failing to explain others, affords strong presumption that there are some fundamental principles of fluid motion of which due account has not been taken in the theory' (Reynolds 1883).

Reynolds revealed this 'fundamental principles' with his pioneering, extremely carefully conducted pipe flow experiments. He used straight, smooth pipes made of glass with different diameters. The working fluid was water and the flow rate and temperature were accurately controlled. In addition he was able to visualize the flow by injecting ink at the entrance of the pipe center. In this experiment he could confirm the onset of turbulence, when the fluid velocity was increased above a critical value (see Fig. 1.3). A systematic continuation of this experiment for different pipe diameters and temperatures lead him to the conclusion, that

'the general character of the motion of fluids [...] depends on the relation between a physical constant of the fluid [the viscosity] and the product of the linear dimensions of the space occupied by the fluid and the velocity' (Reynolds 1883).

This non-dimensional parameter became much later known as the Reynolds number Re (Sommerfeld 1908, Blasius 1911, von Kármán 1954, Rott 1990):

$$
\text { Reynolds number } R e=\frac{u d}{v},
$$

for pipe flow: $u$ averaged velocity, $d$ pipe diameter, $v$ kinematic viscosity. The Reynolds number $R e$ is the most important parameter in fluid dynamics. For low $R e$ flows are laminar, for increasing $R e$ they become turbulent at a critical value. What may look rather simple at a first glance, was a breakthrough in fluid dynamics. The Reynolds similarity principle means that flows on e.g completely different length scales are identical to each other, if $R e$ is the same. This is the basis on which large-scale flows can in principle all be studied in a laboratory experiment.

However, Reynolds realized also that perturbations, e.g. waves in the tank feeding the pipe, changed the critical $R e$ for the onset of turbulence. 


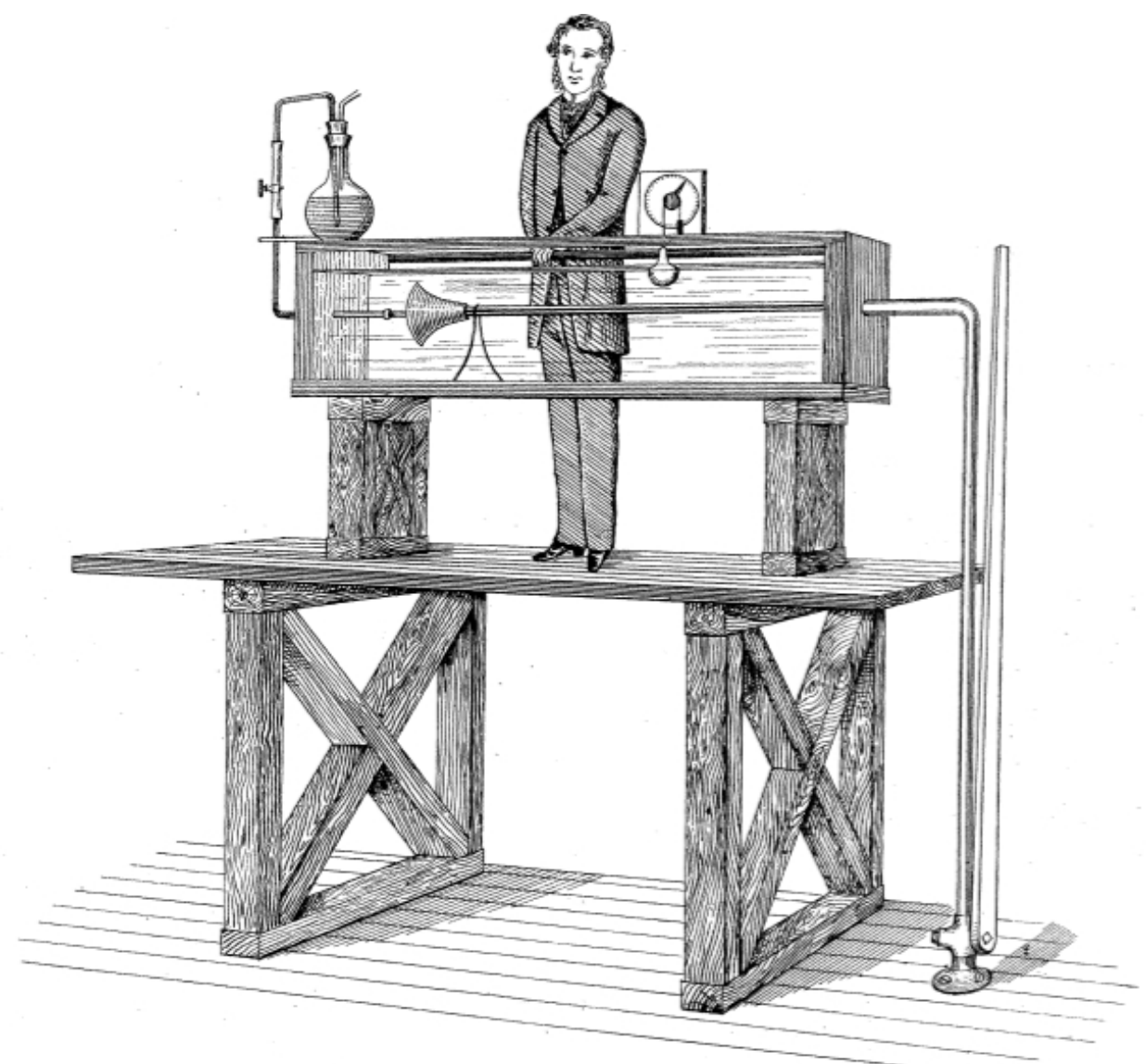

Figure 1.2: Reynolds pipe flow experiment with his assistant. The pipe is made of glass with a diameter of $25 \mathrm{~mm} \pm 0.78 \mathrm{~mm}$ and a length of $1.5 \mathrm{~m}$ and is horizontally placed inside a tank of water. The water from the tank is entering the pipe through a trumpet mouth made of wood. The height of the water in the tank is measured (see instrument to the right side of the assistant) to obtain the velocity of the flow. A vertically mounted iron tube connects the end of the glass pipe with a valve at ground level. A long lever is connected to this valve that reaches up to the platform, so that the flow rate can be easily controlled. For the flow visualization another tube connects a reservoir of ink (placed on top of the water tank) with the centerline of the trumpet mouth. The figure is a public domain and taken from Reynolds (1883). 


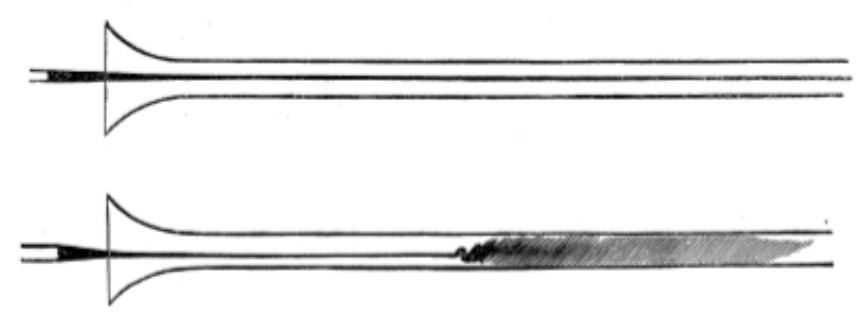

Figure 1.3: Visualization of the onset of turbulence. Ink is added at the center of the trumped mouth. If the flow is laminar, the ink stays confined to the center of the pipe (top picture). The onset of turbulence is not directly at the entrance but some distance downstream. It can be seen by the mixing of the ink with the surrounding water (bottom picture). The figure is a public domain and taken from Reynolds (1883).

'This showed that the steady motion was unstable for large disturbances long before the critical velocity was reached. [...] But the fact that in some conditions it [the laminar flow] will break down for a large disturbance, while it is stable for a smaller disturbance shows that there is a certain residual stability so long as the disturbances do not exceed a given amount' (Reynolds 1883).

What Reynolds was guessing here, is that pipe flow is linearly stable to infinitesimal perturbations and only finite amplitude perturbations can trigger the transition to turbulence. The linear stability was first supported only 100 years later (Salwen et al. 1980, Drazin \& Reid 2004). So far it has been proven to be true at least up to $R e=10^{7}$ in computations (Meseguer \& Trefethen 2003) and up to $10^{5}$ in experiments (Pfenninger 1961). The linear stability of the parabolic velocity profile of pipe flow is the reason, why transition to turbulence is still puzzling researchers nowadays.

But after many experiments Reynolds found a way in which the critical $R e$ for the onset of turbulence could be determined:

'it became clear to me that if in a tube of sufficient length the water were at first admitted in a high state of disturbance, then as the water proceeded along the tube the disturbance would settle down into a steady condition, which condition would be one of eddies or steady motion, according to whether the velocity was above or below what may be called the real critical value' (Reynolds 1883).

Reynolds continued the search for this critical $R e$ for his entire life, even though

'at first sight such experiments may appear to be simple enough, yet when one began to consider actual ways and means, so many uncertainties and difficulties presented themselves that the necessary courage for undertaking them was only acquired after two years' further study of the hydrodynamical aspect of the subject by the light thrown upon it by the previous experiment' (Reynolds 1883). 


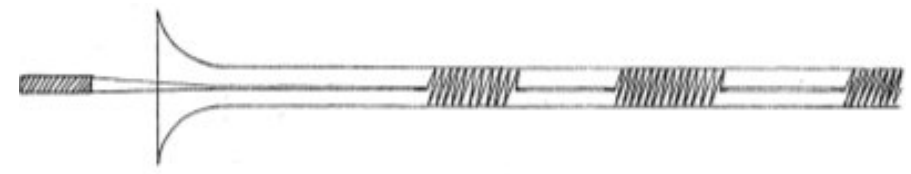

Figure 1.4: Localization of turbulence. At low Re the flow is spatio-temporally intermittent, consisting of an irregular sequence of laminar and turbulent patches in the streamwise direction. Reynolds called the turbulent patches 'flashes of turbulence', nowadays there commonly referred to as 'puffs'. The figure is a public domain and taken from Reynolds (1883).

His last estimates were about $R e=1900,2000$, but he could never get the answer. This is nowadays known as the 'Reynolds-problem'. At these $R e$ turbulence is intermittent, appearing in form of streamwise localized patches that are swept downstream with the surrounding laminar flow as it is illustrated in Fig. 1.4.

\subsection{Transition to turbulence in the 20th century}

\subsubsection{Rotta's pipe flow experiments}

Almost 70 years after the experiments by Osborne Reynold, Julius C. Rotta (1956) quantified how turbulent patches spread while traveling downstream. In his experiments in Göttingen he used glass pipes of different lengths and water as working fluid, but in contrast to Reynolds' experiments the water at the exit of the pipe was ejected as a free jet. The angle of this jet is different for laminar and turbulent flows, which can be explained by the different mean profiles of the velocity (see Fig. 1.7). Rotta used this effect to determine the turbulent fraction of the intermittent flow by placing two containers at different positions (see Fig. 1.5). One container was placed close to the pipe exit thereby collecting the water from a turbulent jet, the other one was located downstream of the first thereby collecting the water from a laminar jet. The ratio of the water volumes of the two containers corresponds to the turbulent fraction at a certain downstream position which corresponds here to the length of the pipe.

From measurements at different pipe length Rotta could infer the corresponding (mean) spreading velocities of the turbulence in terms of the averaged flow velocity for different $R e$. These are shown in Fig. 1.6. It is clearly visible, that the spreading velocities are quickly decreasing for decreasing Re.

'Die Reynoldszahl, bei der sie den Wert Null erreicht, muss als die wirkliche kritische Reynoldszahl angesehen werden, unterhalb derer sich die turbulente Rohrströmung nicht mehr aufbauen kann. Der genaue Wert dieser kritischen Reynoldszahl kann aus vorliegenden Versuchen nicht bestimmt werden; er mag etwa bei Re 2000 liegen' (Rotta 1956).

[freely translated: The Re where the spreading velocity becomes zero, must be regarded as the real critical value. Below it the turbulence cannot be regenerated. The exact value cannot be determined from present experiments, it may be around $R e=2000$. 


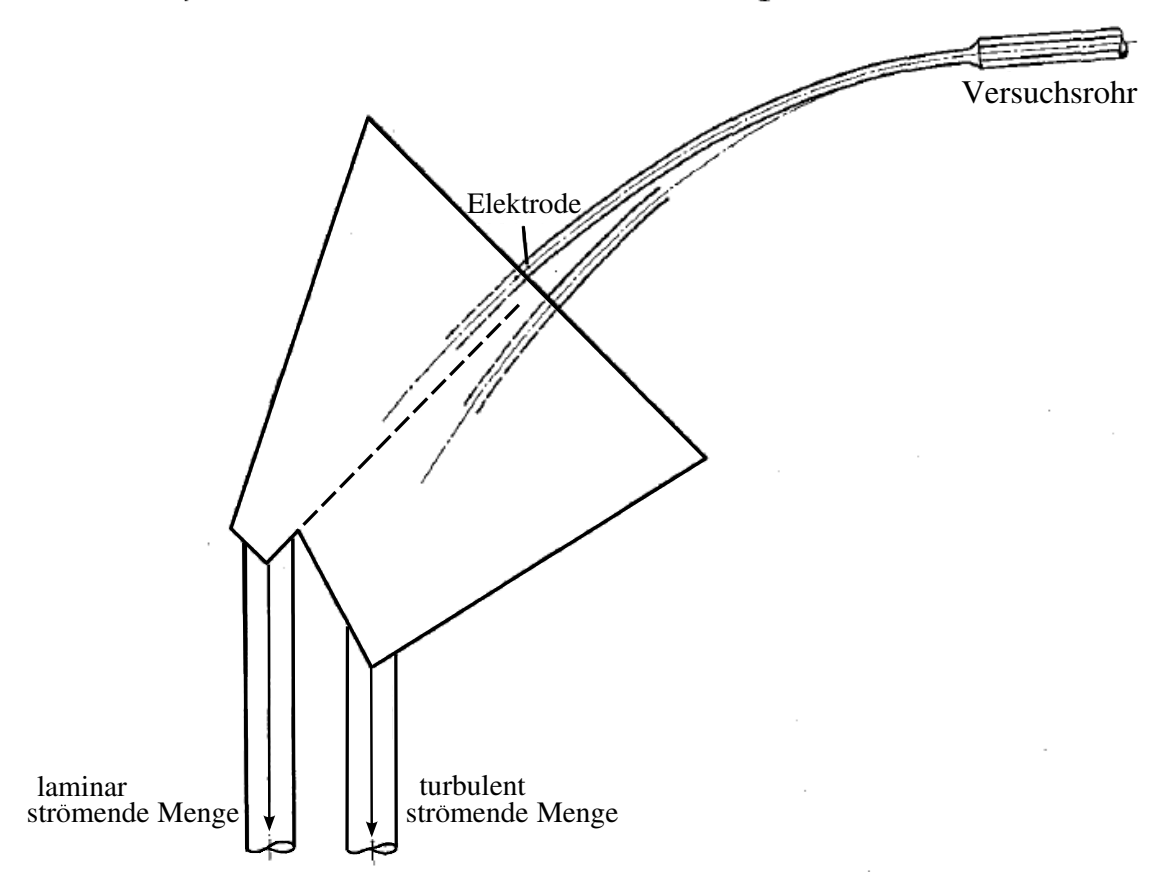

Figure 1.5: Measuring the turbulent fraction. The angle of the free jet of water at the exit of the the pipe is different for laminar and turbulent flows. The left (right) container collects the water from the laminar (turbulent) free jet. The ratio of the measured fluid volumes corresponds to the turbulent fraction. The number of turbulent patches is determined by an electrode, which is positioned in the laminar free jet. The figure is taken from Rotta (1956).

Credit to Ingenieur-Archiv, vol. 24, issue 4, 1965, 'Experimenteller Beitrag zur Entstehung turbulenter Strömung im Rohr', Rotta, page 262, Fig. 4. Reused with kind permission from Springer Science and Business Media.

Despite this clear interpretation, Rotta has been wrongly cited in the literature to have determined the transition to turbulence in pipe flow at $\mathrm{Re}=2300$. Textbooks and even specialized publications (Moxey \& Barkley 2010) commonly give this number by referring to his work. Most likely it is due to his last measurement point in Fig. 1.6 at $\operatorname{Re}=2300$ with a spreading velocity of only $2 \%$.

Another rather common misinterpretation of Rotta's work concerns the flow state that would develop in an infinitely long pipe, called by him 'stationäre Endzustand' [final equilibrium state]. He assumed that the onset of turbulence and the onset of the spreading of turbulence to fill the pipe appears at the same critical Re. Once turbulence has set in it would spread with the velocities shown in Fig. 1.6 until the flow is completely turbulent, i.e. without any laminar gaps ('stationäre Endzustand'). This would apply for any $R e>$ $R e_{c}$.

'Die Partien turbulenter Strömung werden mit der Strömung fortgeführt; dabei wachsen sie mit einer von der Reynoldszahl abhängigen Ausbreitungsgeschwindigkeit und schmelzen nach und nach zusammen, his schliesslich ein voll turbulenter Strömungszustand hergestellt ist. [... ] Dieser Vorgang erstreckt sich über sehr grosse Rohrlängen, die für kleine Reynoldszahlen sogar nach Tausenden 


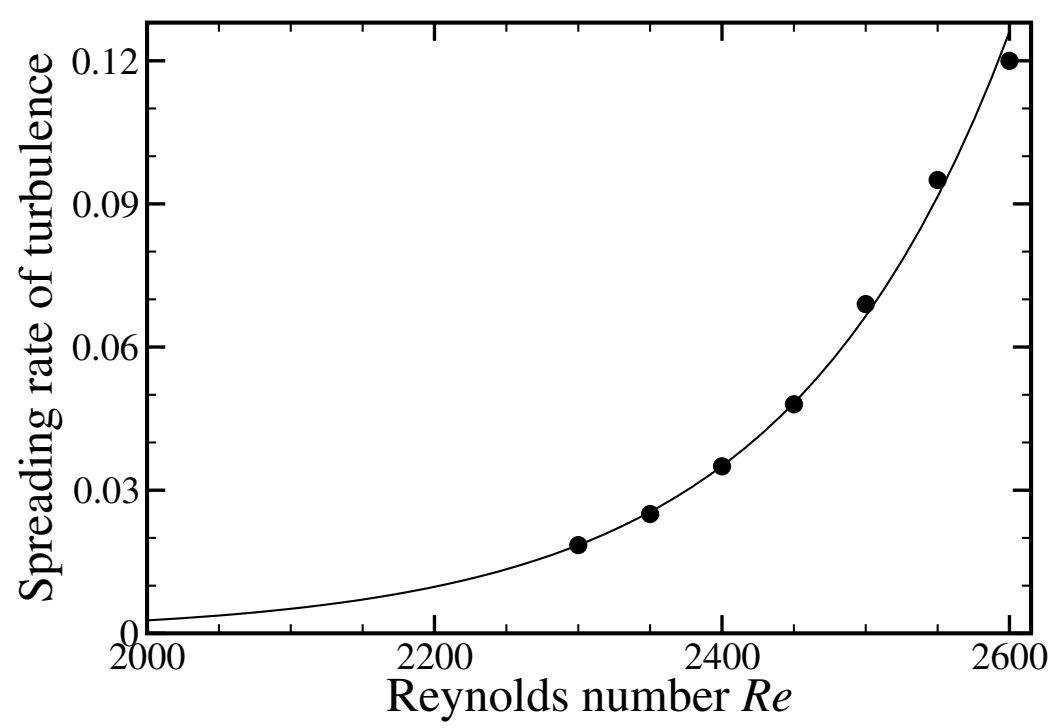

Figure 1.6: Spreading rate of turbulence. Rotta analyzed the turbulent fraction of the flow depending on $R e$ and pipe length. By assuming that the velocity of the turbulent fronts is similar to the mean velocity of the flow the data shown here can be interpreted as the mean spreading rate of turbulence. The line is to guide the eyes. Data are taken from Rotta (1956).

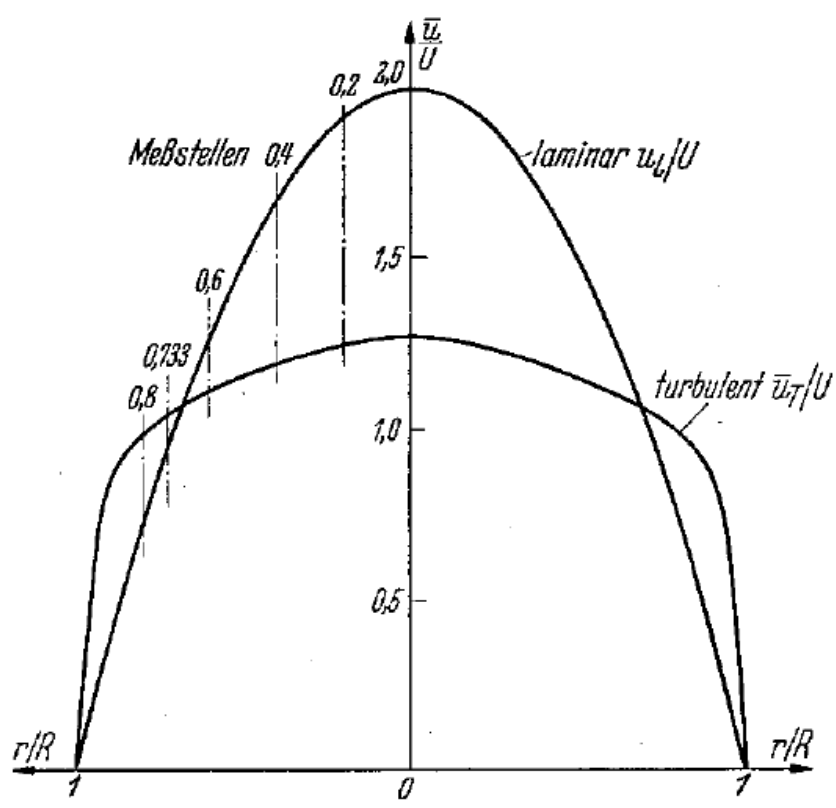

Figure 1.7: Laminar and turbulent velocity profile in pipe flow. The laminar flow profile was mathematically derived by Hagen (1839) and Poiseuille (1840). Hot-wire measurements by Rotta confirmed this 'Poiseuille profile' for laminar flow and showed in addition that turbulence leads (in average) to a plug-like profile. The figure is reproduced from Rotta (1956).

Credit to Ingenieur-Archiv, vol. 24, issue 4, 1965, 'Experimenteller Beitrag zur Entstehung turbulenter Strömung im Rohr', Rotta, page 266, Fig. 9. Reused with kind permission from Springer Science and Business Media. 
von Durchmessern zählen' (Rotta 1956).

[freely translated: Turbulent patches are swept downstream with the mean flow; at the same time these patches spread into the laminar gaps with a spreading velocity depending on the Reynolds number. The final flow state is fully turbulent, meaning that turbulent patches have merged, leaving no laminar gaps in between. [...] This process requires large pipe length, at small Re it can take several thousand pipe diameters.]

To sum up, Rotta was estimating the critical Reynolds number for the onset of turbulence from his extrapolation astonishingly well, but he missed the intermittent character of the turbulence that is intrinsic to pipe flow at low $R e$.

At that time many researchers have tried to derive a mathematical description that can predict the onset of turbulence. For a flow between two plane plates shearing in opposite directions (plane Couette system)

'it seemed probable that the mathematical analysis might prove comparatively simple; but ... it has actually proved very complicated and difficult' (Taylor 1923).

Taylor considered also to investigate pipe flow, but from the experiments of Reynolds he concluded that it is too difficult, because of the finite amplitude perturbations that are required to trigger turbulence. He therefore decided to

'examine the stability of liquid contained between concentric rotating cylinders. If instability is found for infinitesimal disturbances in this case it will be possible to examine the matter experimentally' (Taylor 1923).

The setup used by Taylor was originally invented for a completely different purpose, which is explained in the next section.

\subsubsection{The invention of the Taylor-Couette system and its pioneering studies}

As stated before, the key innovation of the Navier-Stokes equation was to combine the Euler equation with the concept of viscosity. At the end of the 19th century it was therefore the goal of researchers to measure the viscosity precisely in an experiment. Mallock $(1888,1896)$ and Couette (1890) had great engineering skills and built independently from each other the first experiments consisting of two concentric cylinders. The gap between the cylinders was filled with water of different temperatures to determine its viscosity. These were the most precise experiments at that time. Mallock filled even mercury at the bottom of the gap to minimize endwall effects and determined the viscosity of water with an accuracy of $1 \%$ (Mallock 1896, Ritwik 2012). The technique used by Couette of driving the outer cylinder at a constant rotation rate and suspending the inner cylinder on a fibre to measure the torque became later known as Couette viscometers.

Apart from the application of the flow between two rotating cylinders to determine the viscosity of fluids, Rayleigh (1917) used this system for the first time to fundamentally study the stability of rotating flows. In his theory he neglected the influence of viscosity and found that a rotating inviscid flow is in general stable if the angular momentum per Volume $\left(r^{2} \omega\right)$ increases with the radius. By applying this condition on the flow between 


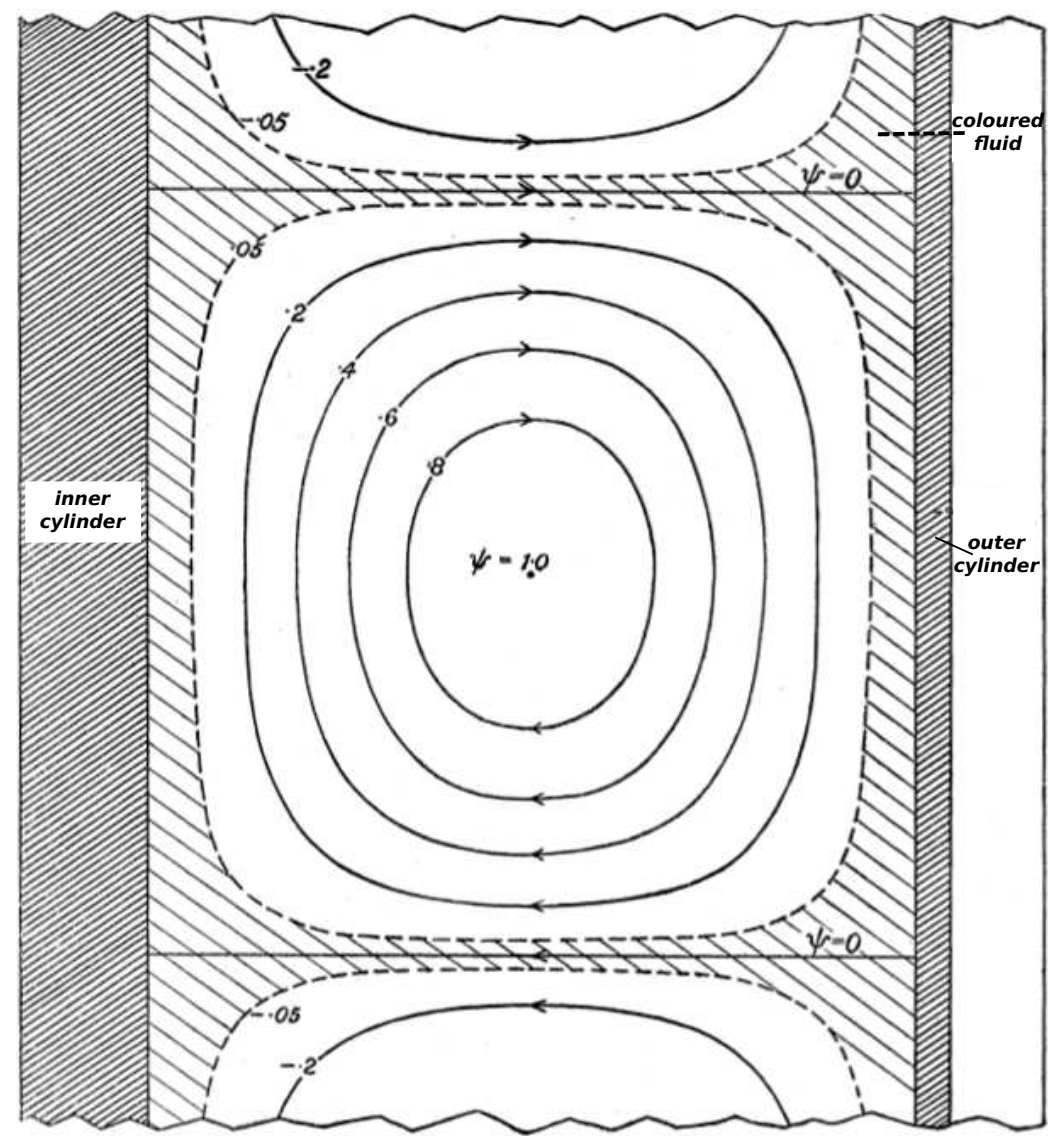

Figure 1.8: Streamlines of the flow instability, calculated by Taylor. The flow pattern is axisymmetric and consists of vortices with a diameter of approximately the gap width. Adjacent vortices rotate in opposite direction. In honor to G.I. Taylor they were later called 'Taylor vortices'. The figure is reproduced from Taylor (1923).

Republished with permission of Phil. Trans. Roy. Soc. Lond., from 'Stability of a viscous liquid contained between two rotating cylinders', Taylor, Vol. 223, page 289-343, 1923; permission conveyed through Copyright Clearance Center, Inc..

two rotating cylinders with radii $r_{i}$ (inner cylinder) $<r_{o}$ (outer cylinder) and the angular velocities $\omega_{i, o}$ respectively, the flow is stable when $\omega_{i} r_{i}^{2}<\omega_{o} r_{o}^{2}$. This condition became later known as Rayleigh criterion.

The flow for a pure inner cylinder rotation (with the outer cylinder at rest) is unstable according to the Rayleigh criterion for all $R e_{i}$. Here $R e_{i}$ is defined as $R e_{i}=\omega_{i} r_{i} d / v$ with the gap width $d=r_{o}-r_{i}$ and the viscosity $v$. But the influence of viscosity can damp instabilities. The exact onset of the instability - including the effects of viscosity - was first investigated by G.I. Taylor experimentally and theoretically in 1923 . He observed in his experiment that the laminar flow is stable for small Reynolds number $R e_{i}$ and becomes unstable when $R e_{i}$ was increased above a critical value $R e_{i, c}$. The laminar flow consists of a purely azimuthal motion, while the instability appears as toroidal vortices. These vortices have a diameter of approximately the gap width $d$ and are alternately rotating in opposite directions as it is illustrated by the streamlines in Fig. 1.8.

What became a breakthrough in fluid dynamics is the fact that Taylor was able to 


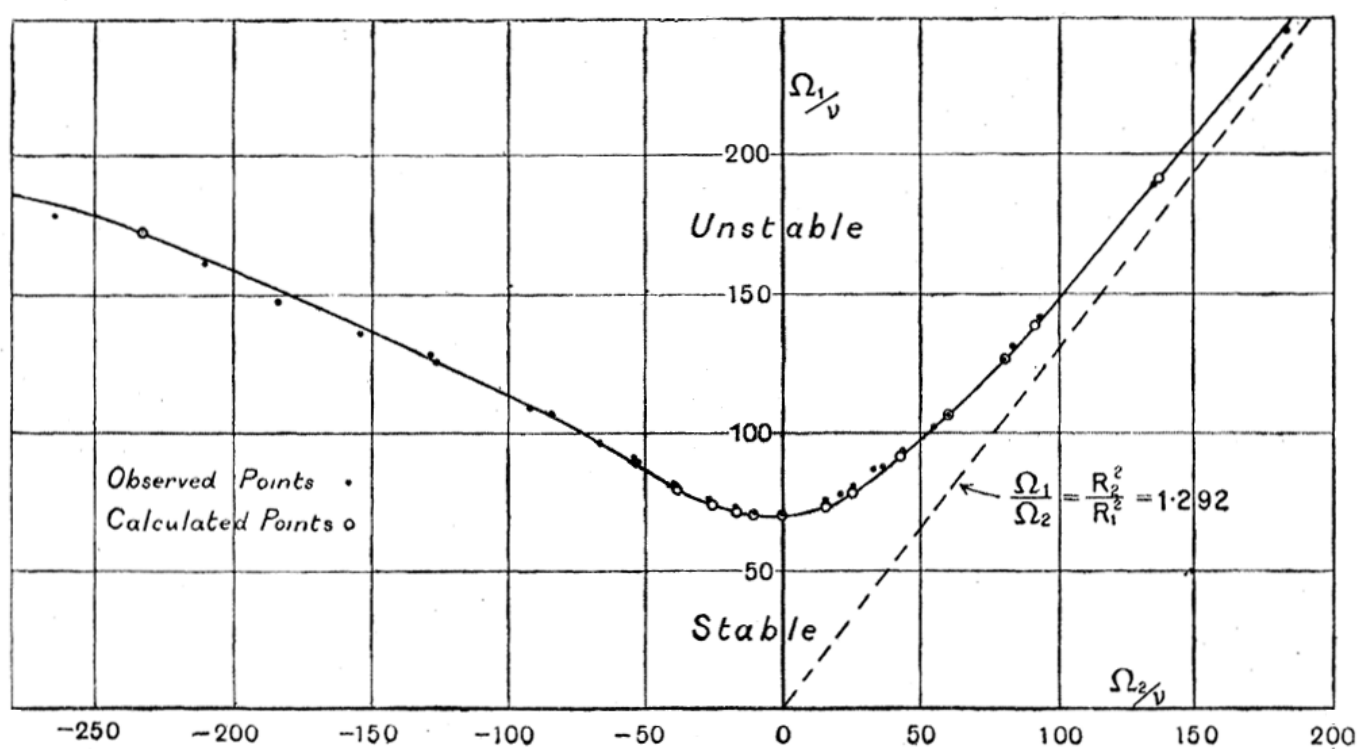

Figure 1.9: Quantitative agreement for the onset of instability between theory and experiment in Taylor-Couette flow. Taylor applied for the first time successfully a linear stability analysis to predict the onset of an instability. The agreement is excellent in all regimes. A pure inner cylinder rotation corresponds to $\Omega_{2}=0$, the co-rotating regime to $\Omega_{2}>0$ and counter-rotating cylinders to $\Omega_{2}<0$. The working fluid was water with a viscosity $v \approx 1 c S t$. The figure is taken from Taylor (1923).

Republished with permission of Phil. Trans. Roy. Soc. Lond., from 'Stability of a viscous liquid contained between two rotating cylinders', Taylor, Vol. 223, page 289-343, 1923; permission conveyed through Copyright Clearance Center, Inc..

predict the exact value of $R e_{i, c}$ from the Navier-Stokes equation. This was the first quantitative agreement between experiment and Navier-Stokes equation, thereby proving the validity of the no-slip condition at the wall. On the other hand to predict the instability he applied a method now called 'linear stability analysis'. This method is nowadays widely used in all fields of physics. The work of Taylor can be regarded as one of the most important one of physics in the 20th century (Donnelly 1991).

In honor of Taylor and Couette the flow between two concentric rotating cylinders is now called 'Taylor-Couette flow' and the axisymmetric toroidal vortices appearing as primary instability are called 'Taylor vortices'.

In the same study Taylor investigated the stability of the flow also for independently rotating cylinders. If the cylinders were co-rotating the onset of instability agreed well with the Rayleigh criterion. But for counter-rotating cylinders only the linear stability analysis could predict the onset of instability for increasing $R e$. The corresponding stability diagram is shown in Fig. 1.9.

\subsubsection{Linear instabilities: supercritical transition}

With the 'linear stability analysis' a certain (flow) state is investigated under the influence of infinitesimal perturbations. Depending on $R e$ and flow state these perturbations can either grow exponentially in time or they decay. If the perturbations grow, the flow state 
becomes unstable to the fastest growing mode. The flow state changes qualitatively. If the perturbations decay, the flow remains the same.

Taylor was able to calculate the primary instability from the laminar flow in a TaylorCouette setup and Chandrasekhar (1961) applied the analysis successfully to the RayleighBenard setup. In this setup the flow is driven by convection between a hot bottom plate and a cold top plate. Note that Rayleigh (1916) had already conducted the stability analysis but with a free-slip boundary condition, which prevented quantitative comparison to experiments. In the following years researchers could also calculate secondary instabilities (Davey et al. 1968, Clever \& Busse 1974), but it was analytically too difficult to analyze further instabilities. From experimental observations it was known, that the flow becomes much more complex and finally turbulent for increasing $R e$. In order to still investigate these flows theoretically, the description switched from a quantitative to a qualitative one. In 1944 Landau proposed that an infinite sequence of instabilities lead to turbulence. The flow would appear 'complicated and confused', but it could still be interpreted as a quasi-periodic flow, consisting of a superposition of many frequencies (Landau 1944, Landau \& Lifshitz 1959, Swinney \& Gollub 1978). A qualitatively different scenario developed Lorenz (1963) almost 20 years later. According to him turbulence is non-periodic and its main characteristic the sensitivity to initial conditions. His theory together with his model laid the foundations of chaos theory that is nowadays extensively used to describe deterministic systems. The dispute could only be settled in the 80s. Ruelle \& Takens (1971) refined the model of Landau and combined it with the chaotic behavior described by Landau. In their scenario only 3-4 instabilities are required and it is the nonlinear interaction of these that give rise to chaotic flows. In phase space this leads to the generation of a so called 'strange attractor' (Ruelle \& Takens 1971). Measurements with a Laser-Doppler Velocimeter confirmed the 'Ruelle-Takens scenario' to turbulence in a Taylor-Couette and Rayleigh-Benard experiment (Gollub \& Swinney 1975). In both experiments the transition from periodic (visible as a single frequency peak in the spectrum) over quasi-periodic (visible as two distinguished frequencies and their linear combinations) to chaotic flow (visible as frequency peaks combined with broadband noise) was clearly detected. I want to highlight that turbulence arises according to Ruelle-Takens exclusively through an increase of temporal complexity, any spatial aspect is neglected here! The expectation behind this theory was that an increasing number of temporal modes would also lead to the development of spatial modes thereby generating the Kolmogorov cascade of turbulence. Many more investigations of such bifurcations and pattern formation followed in Taylor-Couette and Rayleigh-Benard flows and are still topic of current research (Bradshaw 1969, Pfister \& Rehberg 1981, Di Prima \& Swinney 1985, Andereck et al. 1986, Bodenschatz et al. 1991, Dubrulle \& Hersant 2002, Pacheco et al. 2011).

All flows mentioned in this subsection so far have in common that the basic flow becomes linearly unstable to infinitesimal perturbations, thereby giving rise to instabilities. With increasing $R e$ the flow becomes increasingly complex (Ruelle-Takens scenario) at well defined thresholds. This route to turbulence is here referred to as 'super-critical transition' ${ }^{3}$. Part of the success of Taylor's work is based on his choice for the Taylor-Couette flow, which exhibits these features. Many of the previous attempts to calculate the on-

\footnotetext{
${ }^{3} \mathrm{~A}$ specific bifurcation might be still subcritical, but the critical $R e$ is aways well defined.
} 
set of instabilities/turbulence by other researchers were failing, because the flows they investigated were linearly stable to infinitesimal perturbations. Perturbations of a certain strength, finite amplitude perturbations, are here required to trigger the transition. The critical $R e$ is therefore not well defined, but depends on the perturbations. If the perturbation is sufficient, the flow transitions directly from laminar to turbulence. This route to turbulence is commonly referred to as a 'sub-critical transition'. The onset of turbulence in pipe flow is a typical example. We remember that Reynolds observed that the appearance of turbulence depended on the level of perturbations present in his experiments. A nonlinear theory is necessary to understand this transition, which is obviously much more difficult.

\subsubsection{Linearly stable basic flow: subcritical transition}

Reynolds (1883) speculated already that laminar pipe flow is linearly stable and that finite amplitude perturbation are necessary to trigger the transition to turbulence. But also Taylor-Couette flow can be linearly stable for a certain ratio of the cylinder rotation rates. For a pure outer cylinder rotation the flow is Rayleigh-stable for all $R e_{o}$ and hence also linearly stable. Taylor observed for this configuration in his experiment that

'there is a lower critical point below which the flow is in all cases steady, and an upper critical point above which the flow is always turbulent' (Taylor 1936).

These two critical points determine the size of the observed hysteresis. In this hysteretical regime

'the flow is usually stable if the rotation of the outer cylinder is steadily increased through the lower critical point, but a slight disturbance, such as that produced by a slight rotation of the inner cylinder in the opposite direction to that of the outer one, will make the flow permanently turbulent' (Taylor 1936).

Despite this observation and the deep understanding of linear instabilities, it seems that Taylor could not interpret the origin of the upper critical point. Only much later a comparison of this upper critical value from different experiments of that time showed clearly that it depends exclusively on the perturbations present in an experiment (SchultzGrunow 1959, Joseph 1976, Burin \& Czarnocki 2012).

The first systematic study of subcritical turbulence for counter-rotating cylinders was performed by Coles in 1965 . He called this transition 'catastrophic' to emphasize the sudden appearance of turbulence when the laminar flow was disturbed. This transition can be observed when the outer cylinder is rotating faster and in the opposite direction than the inner cylinder. The corresponding flow states consists of a mixture of laminar and turbulent patches. By flow visualization he identified regimes where the turbulent patches seem to be disordered and others where the patches align in a spiral pattern. He defined two thresholds, one below which 'turbulence if present will always decay if left to itself', an intermediate regime where 'turbulence if present will persist indefinitely, but it will not appear spontaneously if the flow is not disturbed' and above the second threshold where linear instabilities set in. The investigation of the intermediate regime 'presents $a$ 
real experimental challenge' and features 'the same properties which distinguish the pipe flow' (Coles 1965). Coles drew here a very important connection between subcritical turbulence in Taylor-Couette flow and pipe flow in general. This resemblance of the dynamics lead to great advances in understanding the nature of this transition by applying an idea or observation from one system to the other.

Coles saw this connection so clearly, because he had worked in pipe flow before. Also here the flow is highly intermittent in space and time at low $R e$. Two different kind of streamwise localized turbulent structures were distinguished at that time. The first one is characterized by a constant length (in average) in the streamwise direction and is called 'puff'. The second one is growing in length and called 'slug'. Coles (1962) found out that puffs and slugs can only be classified by the $R e$ and not by their origin, meaning the kind of perturbation that created them. A far deeper understanding of turbulence in pipe flow at low $R e$ was gained by the experiments of Wygnanski \& Champagne (1973) and Wygnanski et al. (1975). They applied for the first time the concept of a controlled localized perturbation to trigger turbulence (Wygnanski et al. 1975). As working 'fluid' air was used and the perturbation was created by pressure pulses from loudspeakers. The perturbation time and amplitude could be easily changed and, in addition, by placing several hot-wire probes at different streamwise locations the development of the turbulent patches could be followed consecutively while swept downstream with the mean flow. This setup was the milestone for quantitative investigations of the dynamics of turbulence, which is why modern studies are still working with similar methods (Darbyshire \& Mullin 1995, Hof et al. 2003, Durst \& Ünsal 2006, Avila et al. 2011). In all previous studies, turbulence was created randomly in time by placing an obstacle close to the pipe inlet (Reynolds 1883, 1895, Rotta 1956).

In Fig. 1.10 the $R e$-regimes for the appearance of slugs and puffs is qualitatively illustrated. It can be seen that puffs exist at $R e \gtrsim 2000$ and need a higher amplitude of perturbation to be triggered than slugs, appearing for $R e \gtrsim 3000$ (Wygnanski \& Champagne 1973). In fact the required amplitude decreases as a power law over $R e$ (Darbyshire \& Mullin 1995, Hof et al. 2003). In the range of Reynolds numbers where Reynolds and Rotta expected the transition $\left(R e_{c} \approx 2000\right)$, one finds puffs, so that their observations are related to the dynamic of puffs. The so called 'puff splitting' (Wygnanski et al. 1975) is the mechanism through which turbulence spreads in this regime by a spatial proliferation. In this process the puff is temporarily growing in the downstream direction until this 'oversized' puff splits thereby generating a new puff. The two puffs have similar sizes and are swept downstream with the mean flow at an approximately constant distance. The puff splitting is thereby increasing the turbulent fraction of the flow (Rotta 1956, Wygnanski et al. 1975, Moxey \& Barkley 2010, Avila et al. 2011). It was assumed that the spatial proliferation sets in at a critical point, which is why it was often used to predict $R e_{c}$ (Rotta 1956, Wygnanski \& Champagne 1973, Moxey \& Barkley 2010). It is important to note that the appearance of turbulence is here due to spatial proliferation in contrast to the Ruelle-Takens scenario that considers only temporal aspects. 


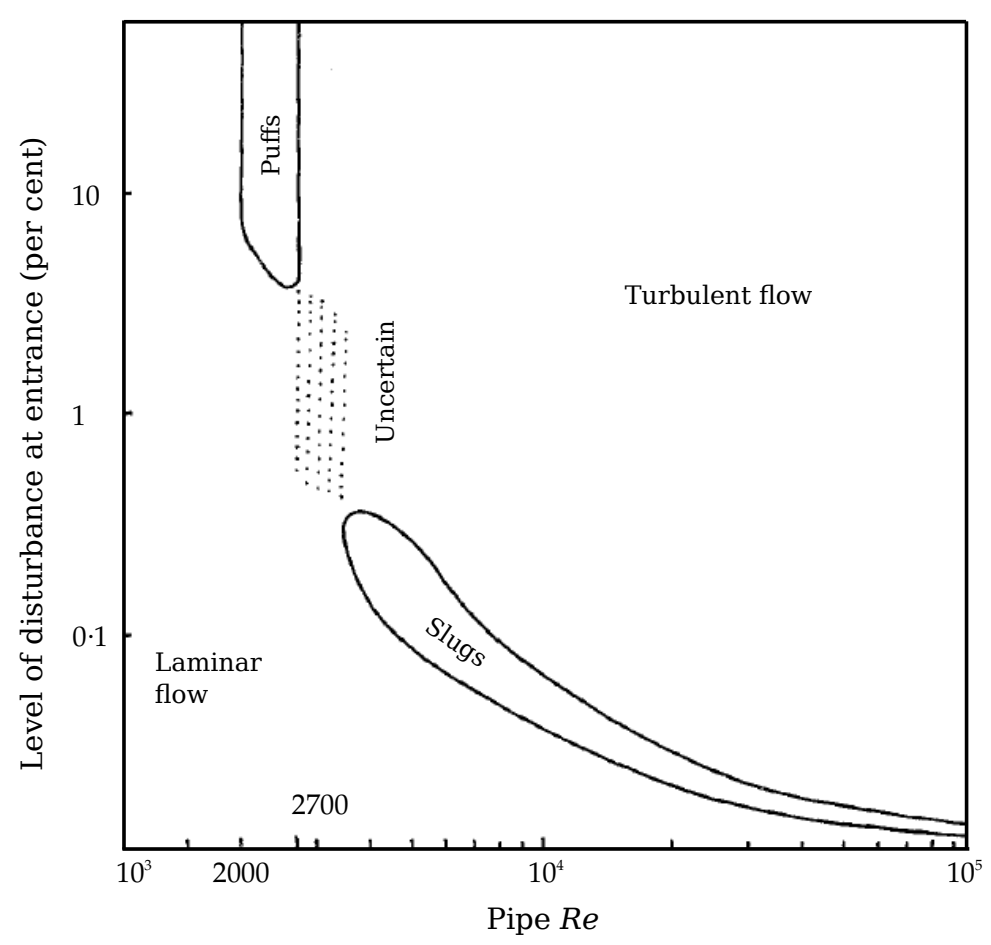

Figure 1.10: Occurrence of puffs depending on Re. Puffs are streamwise localized patches of turbulence with a length of about 20-30 pipe diameters. Slugs are also localized turbulent patches, but their length increases while traveling downstream. The amplitude of the perturbation required to trigger turbulence decreases for increasing $R e$. The figure is taken from Wygnanski \& Champagne (1973).

Republished with permission of Cambridge University press, Journal of fluid mechanics, from 'On transition in a pipe. Part 1. The origin of puffs and slugs and the flow in a turbulent slug', I. J. Wygnanski and F. H. Champagne, Vol. 59, issue 02, page 281-335, 1973; permission conveyed through Copyright Clearance Center, Inc.

\subsubsection{Statistical approach: Directed percolation and Coupled map lattices}

A startling connection between the onset of turbulence in pipes and directed percolation was suggested by Pomeau (1986). The directed percolation model was originally invented to describe the flow of water through porous rocks under the influence of gravitation as it appears in nature (Broadbent \& Hammersley 1957, Hammersley 1957). Here the gravity imposes a preferred spatial direction of the water flow, which characterizes the percolation as directed and not isotropic. The average connectivity of the pores corresponds to the permeability and the global behavior of the system is analyzed by statistical averages. If the permeability is below the critical point the water will (in average) not reach the bottom of the rock, i.e. the rock is impermeable at large scale. If the permeability is above the critical point the water will reach the bottom easily, also over infinitely thick rocks. The critical point corresponds statistically to exactly a single stream of water reaching the bottom. The directed percolation model is a universality class belonging to the group of non-equilibrium phase transitions (Janssen 1981, Grassberger 1982, Hinrichsen 2000a).

The possible connection noted by Pomeau (1986) can only become clear if one looks 


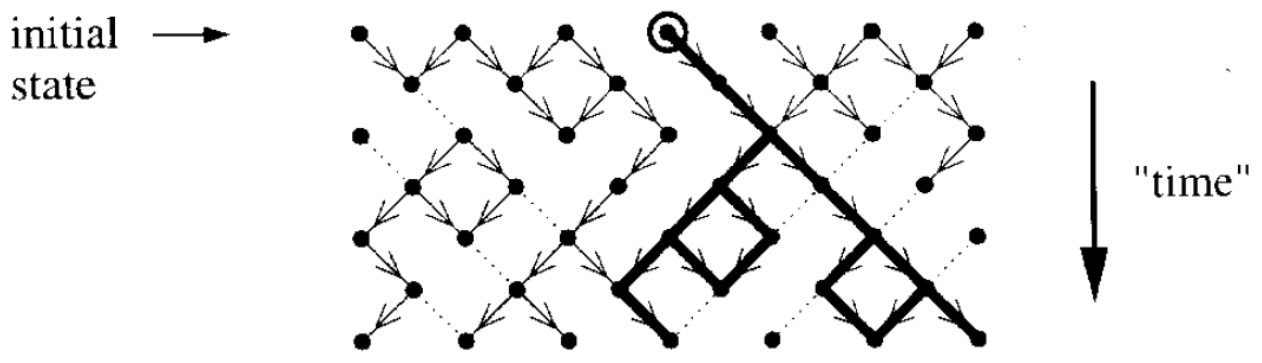

Figure 1.11: Directed percolation model applied to a diagonal square lattice. Open (closed) connection between two sites are drawn as a solid (dashed line). If only the site marked by the circle would be activated as initial condition, the activation would spread along the open bonds and spatio-temporal intermittency, as it is illustrated by the thick lines would appear. The figure is reproduced from Hinrichsen $(2000 \mathrm{~b})$, which is under a Creative Commons Attribution License.

at the directed percolation in a more specific way. In this picture a pore filled with water would be equivalent to a turbulent localized patch (e.g. a puff). Both are described as an 'active site'. A 'passive site' would correspond to a dry pore or a laminar patch. The percolation model describes how the contamination spreads (statistically averaged) from an active site to its neighboring sites depending on the control parameter, or in other words how likely it is that a neighboring site becomes active (see also Fig. 1.11 and Fig. 1.12). In the example of the porous rocks the control parameter is the permeability and the contamination corresponds to the stream of water connecting the pores. In pipe flow the control parameter is the Reynolds number $R e$ and the contamination corresponds to the spreading of turbulence. At a global level, beyond the critical permeability the stream percolates from top to bottom of the rocks. Similarly, beyond the critical $R e$ the turbulence is sustained.

A mixture of active and passive sites corresponds globally to a fluctuating (active) system state. If all sites are passive, the global state is also passive. This passive state must be unique and absorbing: once the system has reached it, the system remains there for all times. A linearly stable laminar flow would be such an absorbing (passive) state. Special requirements are also needed for the fluctuating (active) state, but they are more complicated. The active state can be described by a single parameter, the mean density of active sites.

In porous media this order parameter is the ratio of wet to dry pores, in pipe flow the turbulent fraction. The first requirement out of three to belong to the universality class of directed percolation (Janssen 1981, Grassberger 1982, Hinrichsen 2000a) is that the order parameter changes continuously from the fluctuating (active) to the absorbing (passive) state of the system, as the control parameter is varied. In pipe flow this would correspond to a continuous decrease of the turbulent fraction down to zero (=laminar flow), when the $R e$ is decreased. At the same time this would correspond to a second order nonequilibrium phase transition. The second criteria is the one excluding most systems: only short range dynamics are allowed, similar to a contamination process. In fluid dynamics this means that turbulence can only be generated by a neighboring patch, for example by puff splitting. Turbulence is not allowed to be generated spontaneously from laminar flow 


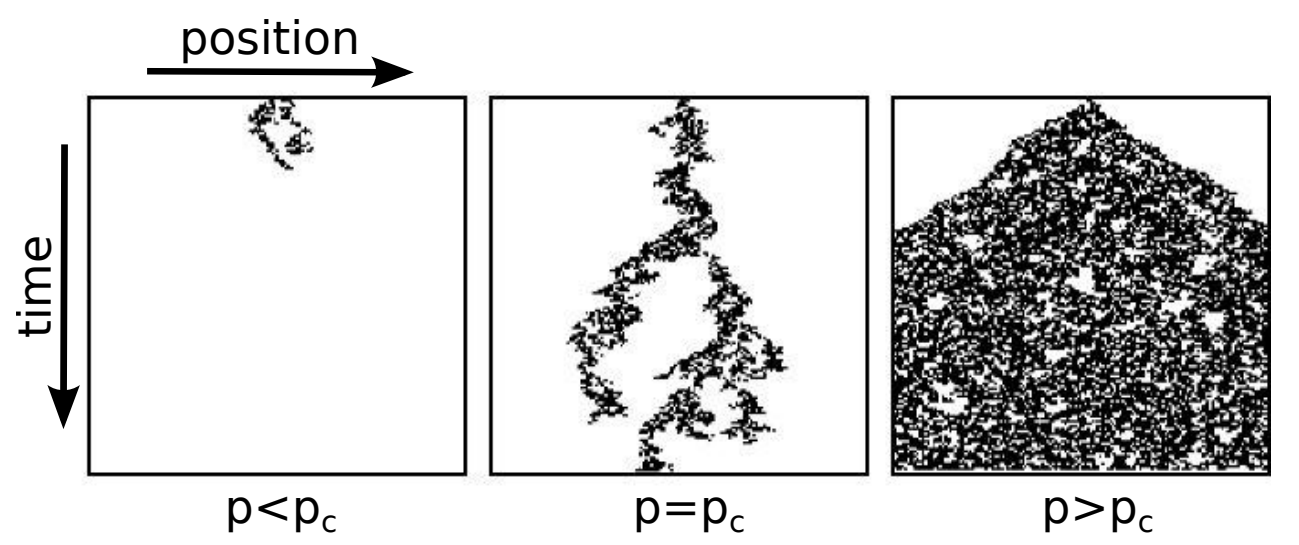

Figure 1.12: Spatio-temporal evolution of active sites below $\left(p<p_{c}\right)$, exactly at $\left(p=p_{c}\right)$ and above $\left(p>p_{c}\right)$ the critical probability $p_{c}$. As initial condition a single site was activated. Applied to pipe flow this would correspond to the triggering of exactly a single puff. Below the critical Reynolds number $R e_{c}$ the puff would most likely decay (left picture), at the critical point it would in average be sustained (middle picture), and for $R e>R e_{c}$ the turbulence would spread (right picture). Similar spatio-temporal dynamics from a direct numerical simulation of pipe flow are shown in Fig. 2.8 for $R e \approx R e_{c}$ and in Fig. 2.2 (B) for $R e>R e_{c}$. The figure is taken from Hinrichsen (2000a) and reused with permission of Taylor \& Francis, 2000.

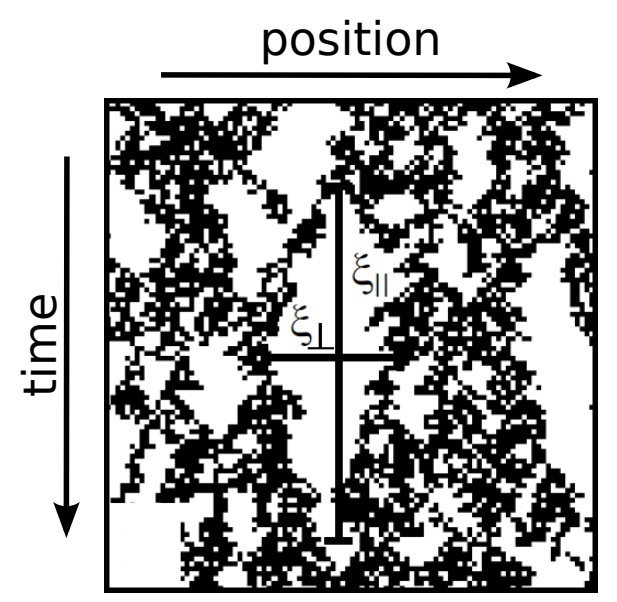

Figure 1.13: Determination of the correlation length $\xi_{\perp}$ and correlation time $\xi_{\|}$from a spatio-temporal pattern. Applied to fluid dynamics, the black (active) sites would correspond to turbulence, the white (passive) regions to laminar gaps. By analyzing the size distributions of the laminar gaps depending on $R e$, the second $\left(v_{\perp}\right)$ and third $\left(v_{\|}\right)$critical exponent can be obtained. Figure taken from Hinrichsen (2000a) and reused with permission of Taylor \& Francis, 2000.

or an underlying instability. The third requirement is about special attributes that should be absent. Such attributes are e.g. additional symmetries or quenched randomness. From pipe and Taylor-Couette flow no such attributes are known. 


\begin{tabular}{lccc} 
scaling & exponent & $1 \mathrm{dim}($ Hinrichsen 2000b) & $2 \operatorname{dim}($ Voigt \& Ziff 1997) \\
\hline$F_{\text {stat }} \propto\left(R e-R e_{c}\right)^{\beta}$ & $\beta$ & 0.2765 & 0.584 \\
$\xi_{\perp} \propto\left(R e-R e_{c}\right)^{-v_{\perp}}$ & $v_{\perp}$ & 1.0969 & 0.734 \\
$\xi_{\|} \propto\left(R e-R e_{c}\right)^{-v_{\|}}$ & $v_{\|}$ & 1.7338 & 1.295
\end{tabular}

Table 1.1: Critical exponents from directed percolation applied to fluid dynamics. The scaling of the turbulent fraction $F_{\text {stat }}$ by approaching the critical point would be described by the first critical exponent $\beta$. The scaling of the correlation length $\xi_{\perp}$ and correlation time $\xi_{\|}$is obtained from the distributions of laminar gaps in space and time and is reflected in the exponents $v_{\perp}$ and $v_{\|}$respectively.

All these requirements seem to be fulfilled for subcritical turbulence and it is an appealing idea to describe the onset of turbulence with this statistical mechanics approach. But these requirements are not sufficient to determine if a system belongs to the directed percolation universality class. This can only be clarified by analyzing the three critical exponents of scaling functions. These are summarized in Table 1.1 and have been numerically estimated (unpublished work of Jensen, mentioned in Hinrichsen (2000b), Voigt $\&$ Ziff (1997)). The first exponent $\beta$ describes the scaling of the density of active states (e.g. the turbulent fraction) with the control parameter (e.g. the $R e$ ). In one dimensional directed percolation close to the transition this exponent is $\beta=0.277$, in two dimensions it is $\beta=0.584$. The scaling follows a power law. For the statistically stationary turbulent fraction $F_{\text {stat }}$ it can be written as $F_{\text {stat }}=\left(R e-R e_{c}\right)^{\beta}$. The second exponent describes the correlation length $\xi_{\perp}$, the third exponent the correlation time $\xi_{\|}$. In fluid dynamics the correlation length $\xi_{\perp}$ is determined from the distribution of the length of laminar gaps appearing in the intermittent flow, as it is illustrated in Fig. 1.13. Far away from the critical point the distribution is exponential, close to the critical point it becomes a power-law with an exponent of $v_{\perp}$. The qualitative change of the distribution can also be seen for the correlation time $\xi_{\|}$. An excellent overview of directed percolation is given by Hinrichsen $(2000 a)$, also pointing out the difficulties of experimental realizations (Hinrichsen 2000b).

It is assumed that directed percolation describes many systems, reaching from the spreading of wood fire over the spreading of diseases. But the experimental confirmation of this theory by observing the corresponding critical exponents was first provided in 2007 for liquid crystals (Takeuchi et al. 2007). Despite many attempts it could up to date not be proven to be a valid description for turbulence (Ciliberto \& Bigazzi 1988, Daviaud et al. 1990, Degen et al. 1996, Colovas \& Andereck 1997, Bottin et al. 1998). But it gave many new ideas to understand the onset of turbulence, which will be explained in the following.

Stimulated by the idea of Pomeau, Chaté \& Manneville (1987) investigated a 1-dim partial differential equation for convective turbulence. They observed spatio-temporal intermittency close to the onset of turbulence. The analysis of the correlation time showed an exponential distribution far away from the critical point and a power-law scaling close to it. But the comparison to directed percolation stayed at such a qualitative description, the corresponding exponent differed substantially from the one of directed percolation. For a better comparison the authors suggested the application of 'coupled map lattices' models, that were invented just two years before by Kaneko (1985). 
Nowadays coupled map lattices are used in various fields of physics. But the motivation to invent them came from turbulence:

'One fascinating idea for the turbulence is to regard it as a direct product state of elementary low-dimensional systems. Landau took a limit cycle as the elementary system and considered a model for the turbulence as a quasiperiodic state with infinite number of incommensurable frequencies, which has been denied by Ruelle and Takens picture and by the abundance of lockings. Then what happens if we choose a low-dimensional chaos as elementary system? This question has led the author to construct and study the 'coupled map lattice' model' (Kaneko 1985).

This model was a milestone for the study of spatio-temporal intermittency in general, although it could not confirm that spatio-temporal intermittency in one dimension belongs to the universality class of directed percolation (Chaté \& Manneville 1988). But Chaté and Manneville still emphasized that a statistical mechanics approach to study turbulence might be very useful.

The first statistical approach to study the onset of turbulence was performed in a plane Couette experiment. This setup consists of two parallel plane plates, shearing in opposite directions. The laminar flow profile is linearly stable for all $R e$ (Romanov 1973, Drazin $\&$ Reid 2004). By applying a localized perturbation in form of a jet turbulence was triggered (Daviaud et al. 1992). At low Re the turbulence decayed and the flow remained laminar. The mean time it takes until the flow relaminarizes is from now on referred to as 'lifetime', and the measurement method as 'lifetime measurements'. Without a perturbation no spontaneous generation of turbulence was observed. Daviaud et al. repeated the procedure up to 10 times $^{4}$. At slightly higher $R e$ they observed a 'contamination' process of turbulent patches that split, merged and decayed - features characteristically for directed percolation. At the same time another group performed a similar investigation in this intermittent regime (Tillmark \& Alfredsson 1992). But the goal of both these studies was the determination of a critical point for the onset of turbulence in plane Couette flow rather than to seek for a connection to directed percolation.

\subsubsection{Statistical analysis of flow dynamics}

The first investigation aiming to analyze the flow dynamics in the transitional regime statistically was performed by Bottin and his collaborators (Bottin et al. 1998, Bottin \& Chaté 1998). They concluded from the previous work in models that

'the spatiotemporal intermittency framework implies that the turbulent regime in plane Couette flow must be approached statistically' (Bottin $\mathcal{F}$ Chaté 1998).

Two different sets of measurements were performed. In the first they measured lifetimes of turbulent spots. But in contrast to the study of Daviaud et al. (1992) they repeated the procedure with about 100 times much more often and found the distribution of the lifetimes to be exponential (see Fig. 1.14). In addition this distribution was independent of

\footnotetext{
${ }^{4}$ The actual number was not mentioned in the original publication by Daviaud et al. (1992), but it is stated in Bottin \& Chaté (1998)
} 


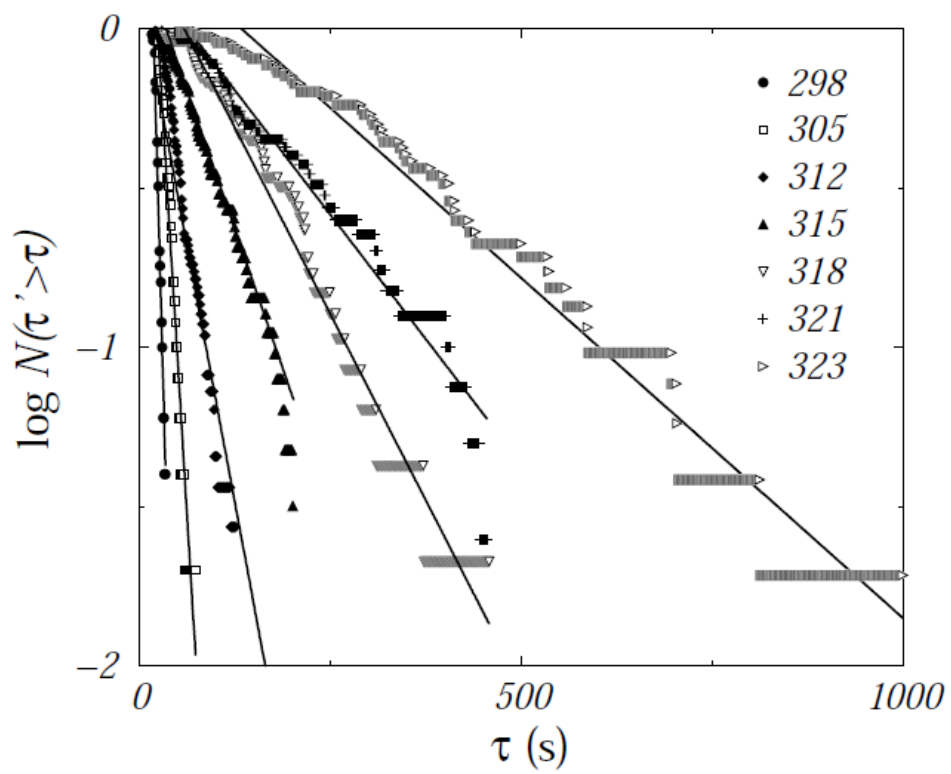

Figure 1.14: First systematic lifetime study in shear flows. In a plane Couette experiment turbulence was triggered and after a sudden decrease in $R e$ the time was measured until the flow relaminarized ('quench experiments'). The measurements were repeated up to 120 times for a single $R e$. An exponential probability distribution was observed, which means that that the probability of a turbulent patch to decay is independent of its age. The slope of a distribution corresponds to the mean lifetime. The figure is taken from Bottin \& Chaté (1998).

Credit to Springer and The European Physical Journal B, vol. 6, 1998, page 143-155, 'Statistical analysis of the transition to turbulence in plane Couette flow', Bottin, Chate, Fig. 6c, in which the material was originally published. Reused with kind permission from Springer Science and Business Media

the kind of perturbation that was applied. With increasing $R e$ the mean lifetime increased rapidly from which the authors inferred a critical $R e_{c}$ above which the lifetimes would diverge and the turbulence would become sustained. Below this threshold the turbulence would be transient. These results indeed revealed for the first time that the onset of turbulence has to be investigated statistically.

In the second set of measurements they analyzed the dependence of the mean turbulent fraction on the $R e$, as it is suggested by the model of directed percolation. But they could not draw a connection to this theory, because they observed a discontinuous first order phase transition. To be able to distinguish between a first and a second order phase transition it is crucial to measure as close as possible to the transition threshold. This would correspond to observe turbulent fractions as close to zero as possible. In the work of Bottin \& Chaté (1998) the minimal mean turbulent fraction was about $35 \%$. However, I note that the system size might be very important in this context (see Chapter 4). If the system size had been larger, it might have been possible to observe smaller turbulent fractions. Many other questions remained open and 
'call for an experimental setup which would allow a more detailed investigation of the threshold region. The most crucial factor in this context is to increase the system size, and we hope that, in the future, carefully controlled experiments with, say, aspect ratios four times larger, will be performed' (Bottin $\mathcal{E}$ Chaté 1998).

This study by Bottin and his collaborators (Bottin \& Chaté 1998, Bottin et al. 1998) was a breakthrough for investigations of the onset of turbulence for flows with a linearly stable basic flow. It has shown that such flows have to be analyzed statistically. The observation that localized patches of turbulence are transient at low $R e$ and that the transition to sustained turbulence can be interpreted as a phase transition are ideas, which still play a key role in research nowadays.

\subsection{Transition to turbulence in the 21 st century}

\subsubsection{Transient turbulence in pipe flow}

In pipe flow transient turbulence was first mentioned by Brosa (1989), who observed the decay of turbulence in numerical simulations. The first systematic statistical investigation of transient turbulence in pipe flow was performed by Faisst \& Eckhardt in 2004. Following the idea of Bottin \& Chaté (1998) they measured lifetimes of large ensembles. The direct numerical simulations were performed in a small box with periodic boundary conditions that captured the local dynamics, but not the localization of the puffs in the streamwise direction. Like in the plane Couette experiments (Bottin \& Chate 1998) they observed an exponential probability distribution and the characteristic mean lifetime increased with $R e$. The results indicated that there was a critical Reynolds number $R e_{c}$ at which the lifetimes would diverge and the turbulence would be sustained (Faisst \& Eckhardt 2004). From the theoretical side the authors connect the onset of turbulence with the generation of a chaotic saddle in phase space. Investigations of the turbulence dynamics indicate a chaotic behavior and the exponential distribution of the lifetimes is characteristic for the escape from such a chaotic saddle. At $R e_{c}$ the divergence of the lifetimes would correspond to a transition from a chaotic saddle to an attractor. But

'the question of whether we will arrive at a turbulent attractor cannot be answered here. But from the rapid increase [of the mean lifetime] it is clear that it will become an attractor for all practical purposes' (Faisst $\mathcal{F}$ Eckhardt 2004).

It was exactly this question that stimulated a series of experiments and further simulations in pipe flow that are summarized in Fig. 1.15. But before explaining them I want to highlight that this investigation of Faisst \& Eckhardt was the first direct numerical simulation (DNS) of the Navier-Stokes equation that contributed substantially to solve the 'Reynolds-problem' of the critical $R e_{c}$ for the onset of sustained turbulence. All such studies before were performed either experimentally or theoretically (including models) 


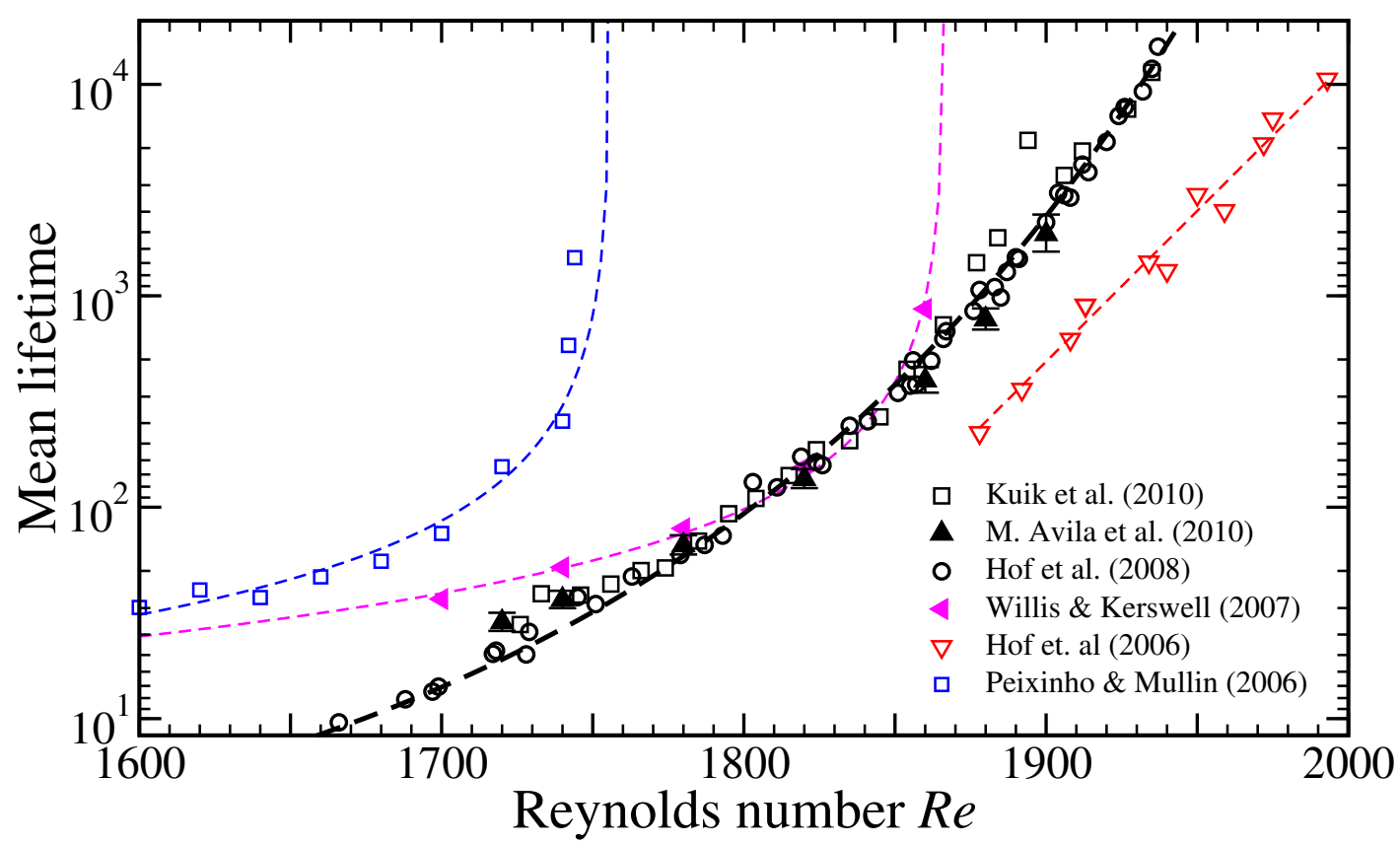

Figure 1.15: Mean lifetime of a puff in pipe flow. Empty symbols correspond to experimentally obtained data, filled symbols to numerical results. The study of Peixinho \& Mullin (2006) and Willis \& Kerswell (2007) suggested a divergence of the lifetimes at a critical $R e_{c}$, which was contradicted by Hof et al. (2006). Experiments (Hof et al. 2008, Kuik et al. 2010) and numerical simulations (Avila et al. 2010) with substantially larger statistical ensembles showed later that the mean lifetime increases super-exponentially and thus does not diverge.

and this study is therefore representative for the inflection point in the investigation of transitional turbulence towards numerical simulations.

The results of Faisst \& Eckhardt (2004) were qualitatively confirmed in experiments by Peixinho \& Mullin (2006). The important invention in the experiment was here that a single puff was triggered and the lifetime of this individual puff detected downstream. A quantitative comparison was not possible due to the small box of the numerical simulations. Although the picture so far seemed to be consistent, it was challenged by Hof et al. (2006) with a new set of experiments in a substantially longer pipe. They obtained transient turbulence far above the $R e_{c}$ that was extrapolated from the previous experiments (Peixinho \& Mullin 2006) and in addition could not confirm a divergence of the mean lifetime. Instead they observed an exponential increase with $R e$. Consequently they followed that turbulence may always be transient. Only

'the rapid exponential increase of lifetimes explains why the transient nature of turbulence has not been observed previously: to detect the decay of turbulence in a garden hose at a flow rate as low as $1 \mathrm{l} / \mathrm{min}(\mathrm{Re}=2400)$ would require a physical length of the tube of $40000 \mathrm{~km}$, about the Earth's circumference, and an observation time of almost 5 years' (Hof et al. 2006). 
The dispute continued with a numerical study (Willis \& Kerswell 2007) in long domains that supported the divergence of the lifetimes at a $R e_{c}$, but showed a shift in $R e$ of about +100 compared to the experiments by Peixinho \& Mullin (2006) and a shift of approximately -30 with the experiments of Hof et al. (2006). A new set of experiment by Hof et al. (2008) increased the investigated time scales by 6 orders of magnitude and revealed a superexponential scaling of the mean lifetimes with $R e$ and supported thus the transient nature of turbulence. Compared to all previous measurements the experiments were conducted much more carefully and the measurement method was automatized, thereby making it possible to collect much larger statistics. The dispute was finally settled when DNS results by Avila et al. (2010) agreed quantitatively with the new results of Hof et al. (2008). The simulations were performed as in Willis \& Kerswell (2007), but they were statistically much better resolved and reached up to higher $R e$. The $95 \%$ confidential intervals of the lifetimes detected by DNS reflect the statistical uncertainty and are shown in Fig. 1.15. All these studies demonstrate how difficult it is to quantitatively describe flows like pipe flow. The timescales involved are much larger than previously anticipated. No critical $R e_{c}$ could be detected up to $10^{8}$ advective units (Hof et al. 2008) and the superexponential scaling of the mean lifetimes indicates that such a threshold does not exist. Turbulence seems to be always transient (Hof et al. 2008, Avila et al. 2010). Experimentally the different results and shifts in $R e$ of the studies also indicate how difficult it is to determine the exact Re (Peixinho \& Mullin 2006, Hof et al. 2006).

In flows that become linearly unstable, like Taylor-Couette flow, a quantitative agreement between theory and experiment was already proven almost 90 years ago (Taylor 1923). On the other hand the numerical study by M. Avila et al. (2010) achieved the first quantitative agreement between simulation and experiments of flows without a linear instability in the transitional regime. The experiments by Hof et al. (2008) were experimentally confirmed in another study (Kuik et al. 2010) and can now be used as a benchmark to check the precision in $R e$ of a pipe flow experiment. On the theoretical side the picture emerges that localized turbulence in pipe flow at least in this intermittent regime is described by a strange repeller in phase space (Hof et al. 2008, Avila et al. 2010).

\subsubsection{Spatial proliferation of turbulence in pipe flow}

The concept of spatio-temporal intermittency (Kaneko 1985) was introduced to pipe flow by Moxey \& Barkley in 2010:

'While it is possible that all turbulence in pipe flow is transient, it does not seem likely. It seems equally unlikely that lifetime measurements of localized puffs will ultimately determine a critical Re. We believe that the key to the transition to sustained turbulence is not in the lifetimes of localized puffs, but in the spatio-temporal aspects of the turbulence' (Moxey $\mathcal{E}$ Barkley 2010).

The 'spatio-temporal aspects' of their investigation were focused on the spreading of turbulence in the transitional regime. Rotta (1956) already quantified the spreading of turbulence, but he missed the intermittent character of turbulence at this $R e$ and assumed that the flow would become fully turbulent for all $R e>2000$. Wygnanski et al. (1975) captured the intermittent character of turbulence and investigated in detail how localized turbulence spread via the 'puff splitting', but they were not aware of the transient nature 
of puffs. Moxey \& Barkley (2010) were the first to compare the purely temporal behavior of puffs, which is reflected in the lifetimes, with their spatial dynamics leading to a spread of turbulence. At $R e_{c}$ the spreading of turbulence (called 'contamination') occurs

'vastly faster than the characteristic lifetime for decay of a puff. As is well established for directed percolation, once the probability ratio of contamination to decay exceeds a critical value, turbulence has a finite probability of sustaining indefinitely as spatial-temporal intermittency, even though any individual turbulent patch has a finite probability of decay. Thus there is a clear mechanism, involving spatio-temporal intermittency, that implicates a change to finite probability of indefinitely sustained turbulence above' $R_{c}$ (Moxey E Barkley 2010).

The idea to compare the timescales of the decay of puffs with the timescales of the spreading of them to determine the onset of sustained turbulence in pipe flow was the leading idea to solve the Reynolds problem. However, in this numerical study it was assumed that the spreading of turbulence is a deterministic process and can therefore be investigated with a single simulation. My experimental results in Chapter 2 show that this assumption was wrong. The spreading of turbulence is a stochastic process and only by analyzing the mean time of its occurrence and comparing it to the mean time of the decay, the critical $R e_{c}$ Reynolds was looking for could be determined to $R e_{c}=$ $2040 \pm 10$. Many critical $R e_{c}$ have been determined in previous studies, often relying on newly introduced concepts. In order to not confuse the reader, in this introduction only the values of $R e_{c}$ estimates that were close to the one determined in this thesis have been listed. The values reported in previous studies reach from 1700 to typically 2300 or even up to 6000 (Darbyshire \& Mullin 1995, Faisst \& Eckhardt 2004, Eckhardt 2009, 2008), but I am convinced that finally we found the 'real critical value'(Reynolds 1883).

\subsubsection{Plane Couette flow: 1st or 2nd order phase transition?}

Bottin \& Chaté (1998) suggested in their cutting-edge study to build a plane Couette experiment with a larger system size to allow for more detailed studies close to the critical point. French researchers (Prigent et al. 2003) experienced in plane Couette flow worked for about five years on a setup that was almost 10 times larger than the one previously used (Bottin \& Chate 1998), but even after such an effort the accuracy of the $R e$ was still 'at best 7.5\%' (Prigent et al. 2003). The problem here is that a large system size corresponds to a small gap between the plates, which is mechanically difficult to maintain constant over the whole surface of the plates. The achieved accuracy was not enough for studies close to the critical point.

Another problem are the large time scales involved. In the experiments the observation time was up to $1.0 \cdot 10^{6}$ advective units to reach the statistically stationary flow state (e.g. turbulent fraction) (Bottin et al. 1998, Bottin \& Chaté 1998). However, this is not durable for DNS even in recent years. Duguet et al. (2010) calculated the mean turbulent fraction of a large system ( $800 \times 365$ half gap width), but the simulations were stopped after 2.0 . $10^{4}$ advective units. Since the time scales of the flow to equilibrate are increasing when the critical point is approached ('critical slowing down'), Duguet et al. were not able to come as close to the critical point as the experiments (Duguet et al. 2010, Bottin et al. 1998). 


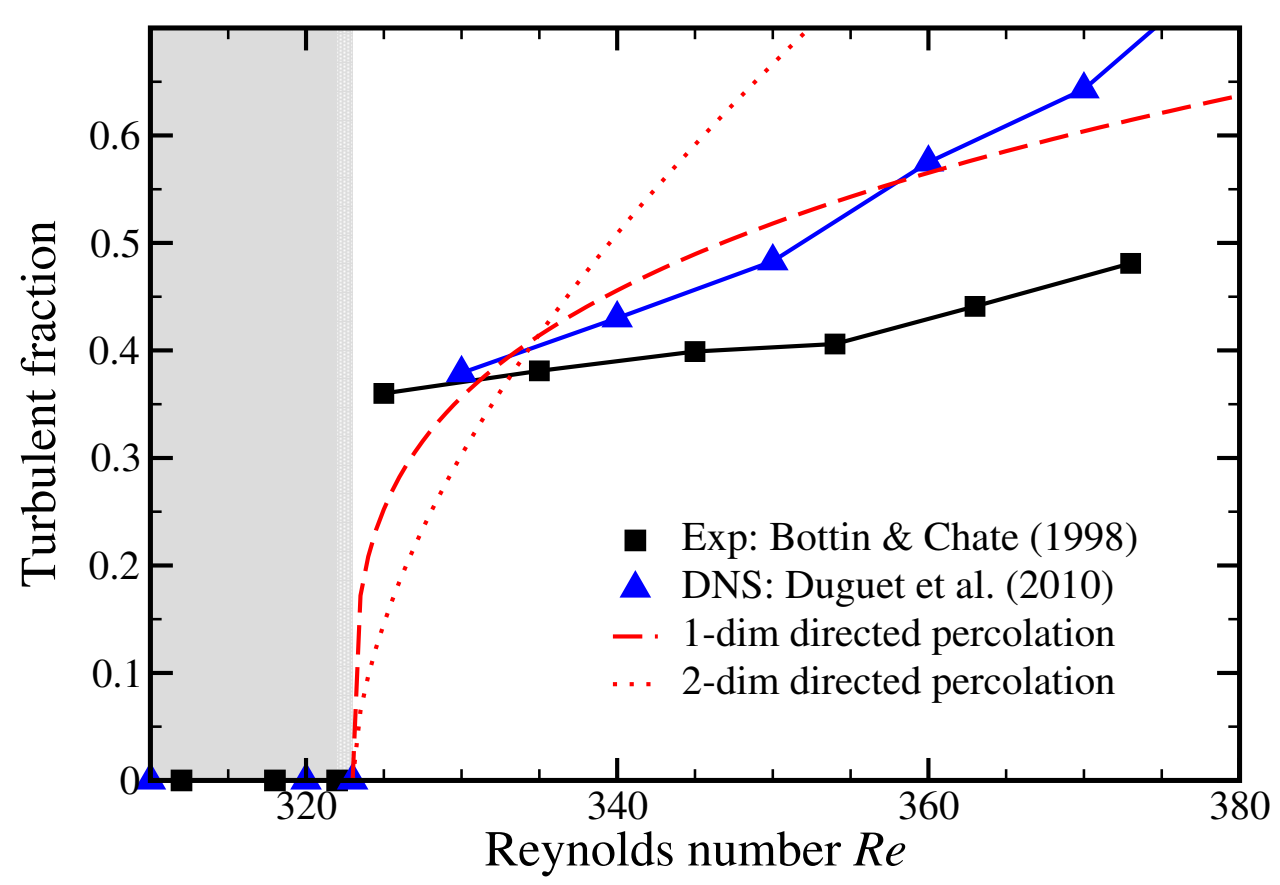

Figure 1.16: Phase transition in plane Couette flow. In the gray shaded regime turbulence is transient. Above a critical $R e_{c} \approx 323$ the turbulence is sustained. In this regime the mean turbulent fraction was determined experimentally (Bottin \& Chaté 1998) and numerically (Duguet et al. 2010). Both studies interpret their results as a first order phase transition. A comparison of the numerically obtained data with scalings corresponding to directed percolation indicates that a second order phase transition may be compatible with these results.

Experiments and numerical simulations agree qualitatively and Duguet et al. interpreted their results as a confirmation of a discontinuous first order phase transition. However, a direct comparison of these simulations with the scaling corresponding to one or two dimensional directed percolation as I have plotted it in Fig. 1.16 reveals that the numerical results cannot distinguish between a first and a second order phase transition, because they are too far away from the critical point. Manneville investigated this transition with a model of the Navier-Stokes equation and found that it

'could be second order (continuous) or first order (discontinuous). Experiments tell us that, in the cases studied so far, it is first order and thus deprived of any universality (correlation lengths remain finite at threshold)' (Manneville 2009).

The studies on the phase transition in plane Couette flow can be summarized as follows:

1. The dynamical behavior of the flow resembles that of directed percolation (which is a 2 nd order phase transition) and all requirements mentioned by Hinrichsen (2000a) to belong to this universality class are fulfilled.

2. Models allow for a 1st and a 2nd order phase transition (Manneville 2009). 
3. Direct numerical simulations (Duguet et al. 2010) cannot resolve the time scales and can therefore not distinguish between 1st and 2nd order transition.

4. Experiments seem to clearly observe a 1st order phase transition (Bottin et al. 1998, Bottin \& Chate 1998). However, the relatively small system size and the deviation of the $R e$ on the order of $3 \%$ may change the transition qualitatively. Further explanations are given in Chapter 4.

\subsubsection{Taylor-Couette flow: Analogy and differences to plane Couette flow}

The first attempt to determine the phase transition in Taylor-Couette flow was performed in 1997 by Colovas and Andereck, one year before the study in plane Couette flow by Bottin et al. (1998). In these experiments a continuous (linear) decrease of the turbulent fraction was observed, but the scaling of the distribution of laminar domains followed a power-law also far away from the critical point. From directed percolation a crossover from a power-law scaling to an exponential one would be expected for an increasing distance from the critical point (Chaté \& Manneville 1987). The problem in this investigation were the underlying long-range instabilities. In contrast to plane Couette flow, which is linearly stable for all $R e$, Taylor-Couette flow becomes linearly unstable to instabilities depending on the rotation rates of the cylinders and their radius ratia. The investigation of Colovas \& Andereck (1997) was performed in the linearly unstable regime. The 'laminar flow' was not a purely azimuthal motion, but a laminar spiral mode, which triggered turbulent bursts that grow in size for increasing $R e$. The turbulent fraction in this study refers to the ratio of the turbulent bursts to the laminar spirals. However, the laminar spirals are long-range coherent structures, so that the flow does not fulfill the requirements for directed percolation (Hinrichsen (2000a) and section 1.2.5). It can therefore be expected that the transition does not belong to the directed percolation universality class.

The radius ratio $\eta$ of the cylinders plays an important role to connect the Taylor- and the plane Couette system as it is illustrated in Fig. 1.17. The plane Couette system corresponds to $\eta=1$ and the laminar flow is linearly stable for all $R e$. As $\eta$ approaches one in the Taylor-Couette system, the primary instability are still Taylor vortices but the secondary wavy vortex state bifurcates subcritically and can be traced to lower Re. Faisst $\&$ Eckhardt (2000) converged this wavy mode into a flow state in the plane Couette system (e.g. the Nagate-Busse-Clever flow state (Nagata 1990, Clever \& Busse 1997)) by increasing $\eta$. In addition, they identified a parameter regime at large $\eta$, in which the flow is linearly stable and the turbulence decays in some cases (Faisst \& Eckhardt 2000). A more detailed analysis of lifetime measurements in Taylor-Couette flow was performed experimentally a decade later by Borrero-Echeverry et al. (2010). For a pure outer cylinder rotation the flow is linearly stable and the turbulence decays exponentially, exactly like in plane Couette (Bottin et al. 1998) or pipe flow (Faisst \& Eckhardt 2004). The experiments have further shown that the mean lifetimes of the turbulent spots remain finite and do not diverge.

These investigations show that pipe, plane Couette and Taylor-Couette flow have similarities beyond that of qualitative resembles that Coles observed already in 1965. More similarities between plane and high radius ratio Taylor-Couette flow were found by Pri- 
(a)

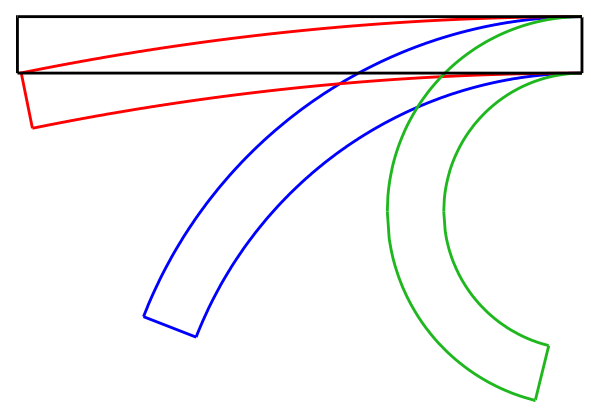

(b)

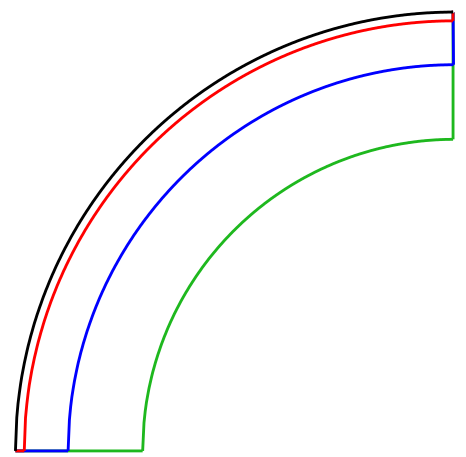

Figure 1.17: Radius ratio dependences. Three different radius radii are displayed and indicated by color: $\eta=0.71$ (green), $\eta=0.88$ (blue) and $\eta=0.98$ (red). (a) The influence of the radius ratio $\eta$ on the curvature for azimuthal length $L_{a z i m}=10$ gap width is illustrated in comparison to a plane Couette setup ( $\eta=1$, black). (b) In experiments different $\eta$ are realized by exchanging e.g. the inner cylinder, while maintaining the outer cylinder (indicated by the black line). An increasing $\eta$ leads to a decreasing gap width. The smaller the gap compared to the radii, the more difficult it is to reach a high accuracy gap width. The radius radii displayed are used in the Taylor-Couette experiment built in this thesis (see Chapter 3).

I thank M. Avila for preparing these sketches for me. A sketch similar to the one in (a) is shown in Faisst \& Eckhardt (2000).

gent et al. (2002). In their experiments they observe a quantitative agreement between the axial wavelength of spiral-turbulence in the Taylor-Couette system and the transversal wavelength of turbulent bands in the plane Couette system (see Fig. 1.18). However, there were also clear differences between these flows, which are explained in the following. The onset of turbulence in plane Couette flow depends on the amplitude of the perturbations. If no controlled perturbation is specifically added, the flow becomes turbulent at an upper limit of $R e_{u}$, which depends on the precision of the setup. If the $R e$ is decreased afterward, the flow relaminarizes at a lower limit $R e_{l}$. The value of $R e_{l}$ depends on the lifetime statistics, but in general $R e_{l} \ll R e_{u}$. This means the flow features a strong hysteresis as it was already observed by Coles (1965).

However, such a hysteresis was not observed in the Taylor-Couette experiments by Prigent \& Dauchot (2005). In the Taylor-Couette system the hysteresis is typically between the linear instability as the upper $R e_{u}$ and the subcritical transition at the lower $R e_{l}$ where perturbations are able to trigger turbulence (Coles 1965). The main difference between these two Taylor-Couette experiments is the radius ratio $\eta$. In the study by Coles $\eta=0.881$, while in the experiment by Prigent \& Dauchot $\eta=0.983$. From the theoretical side a larger $\eta$ would lead to a larger hysteresis (Faisst \& Eckhardt 2000), which couldn't be observed by Prigent \& Dauchot (2005). A hint to the reason why no hysteresis was observed is given by an experimental study in an even larger radius ratio $\eta=0.99$ (Carey et al. 2007). There, the onset of Taylor vortices for a pure inner cylinder rotation could only be detected with an accuracy of $20 \%$ in $R e$, most likely due to local variations of the gap width. In the subcritical regime such deviations in the gap width may act as finite amplitude perturbations and trigger the transition to turbulence. In the plane Couette experiments, it was exactly the uncertainty in the gap width that prevented the application 
(a)

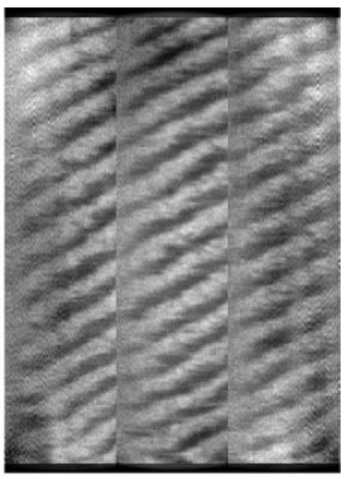

(b)

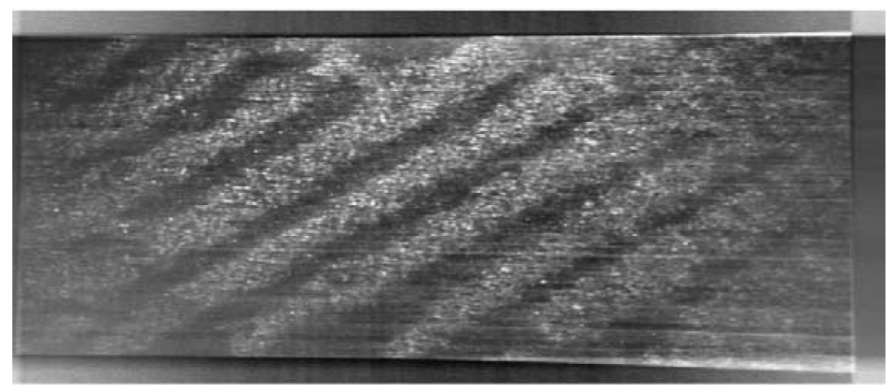

Figure 1.18: Intermittent turbulence in a Taylor-Couette (a) and a plane Couette (b) experiment. In Taylor-Couette flow this pattern is called 'spiral' or 'barber pole turbulence'. The usage of mirrors allowed a $360^{\circ}$ view of the flow, as it is shown in this picture. The similarities go beyond that of a qualitative one. Comparisons of the wavelength (Prigent et al. 2002) and of lifetime dynamics (Bottin et al. 1998, Borrero-Echeverry et al. 2010) have shown this. The figures are taken from Prigent et al. (2003).

Reprinted from Physica D, vol. 174, no. 1, Prigent, Grégoire, Chaté, Dauchot, 'Long-wavelength modulation of turbulent shear flows', pages 100-113, 2003 with permission from Elsevier.

of larger system size setups to study the phase transition (Bottin \& Chate 1998).

These difficulties could be overcome in the new high-precision Taylor-Couette setup that was developed within this thesis and is presented in Chapter 3. The measurements reveal the existence of a large hysteresis, thereby providing the possibility to study the phase transition in an 'unperturbed' environment. The setup has a system size 12 times larger than the plane Couette experiments used before (Bottin et al. 1998, Bottin \& Chaté 1998). Indeed the measurements in Chapter 4 show that the phase transition is of second order, which is in contrast to previous work in plane Couette flow (Bottin et al. 1998, Bottin \& Chaté 1998, Duguet et al. 2010). 


\section{The onset of turbulence in pipe flow}

Citation and credit: This is the author's version of the work. It is posted here by permission of the AAAS for personal use, not for redistribution. The definitive version was published in Science on volume 333, number 6039, page 192-196, 8th July 2011. DOI: $10.1126 /$ science. 1203223

Title: 'The onset of turbulence in pipe flow'

Authors: K. Avila ${ }^{1}$, D. Moxey ${ }^{2}$, A. de Lozar ${ }^{1}$, M. Avila ${ }^{1}$, D. Barkley ${ }^{2,3}$ and B. Hof ${ }^{1}$ ${ }^{1}$ Max Planck Institute for Dynamics and Self-Organization, 37073 Göttingen, Germany

${ }^{2}$ University of Warwick, Coventry, United Kingdom

${ }^{3}$ PMMH (UMR 7636 CNRS - ESPCI - Univ Paris 06 - Univ Paris 07), 10 rue Vauquelin, 75005 Paris, France

Contribution: I built the experiment, performed the measurements ${ }^{1}$, analyzed the corresponding data and prepared most of the figures (Fig. 2.1A-B, Fig. 2.3, Fig. 2.4, Fig. 2.5, Fig. 2.6, Fig. 2.7, Fig. 2.9, Fig. 2.10) for publication. I wrote the first draft, whose structure has been preserved. The work is a combination of experiments (K. Avila, Lozar, Hof) and numerical simulations (Moxey, M. Avila, Barkley). The main message of this publication (determination of the critical point) is based on the experiments I performed and analyzed.

\subsection{Abstract}

Shear flows undergo a sudden transition from laminar to turbulent motion as the velocity increases and the onset of turbulence radically changes transport efficiency and mixing properties. Even for the well-studied case of pipe flow, it has not been possible to determine at what Reynolds number the motion will be either persistently turbulent or ultimately laminar. We show that in pipes, turbulence that is transient at low Reynolds numbers becomes sustained at a distinct critical point. Through extensive experiments and computer simulations we are able to identify and characterize the processes ultimately responsible for sustaining turbulence. In contrast to the classical Landau-Ruelle-Takens view that turbulence arises from an increase in the temporal complexity of fluid motion, here, spatial proliferation of chaotic domains is the decisive process and intrinsic to the nature of fluid turbulence.

\footnotetext{
${ }^{1}$ all except for two in Fig. 2.5 where the perturbation was created by an obstacle
} 
(a)

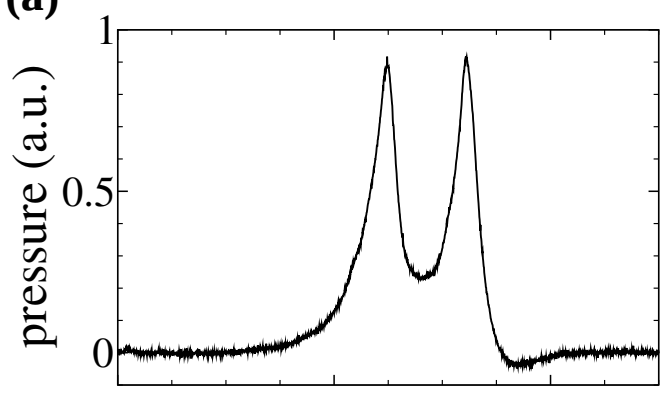

(b)

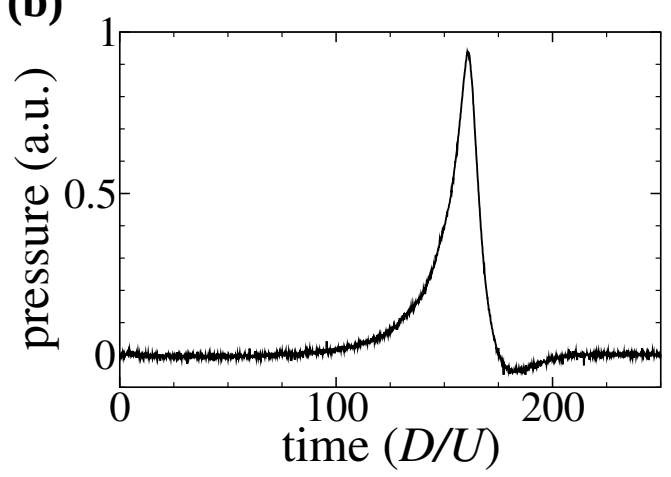

(c)

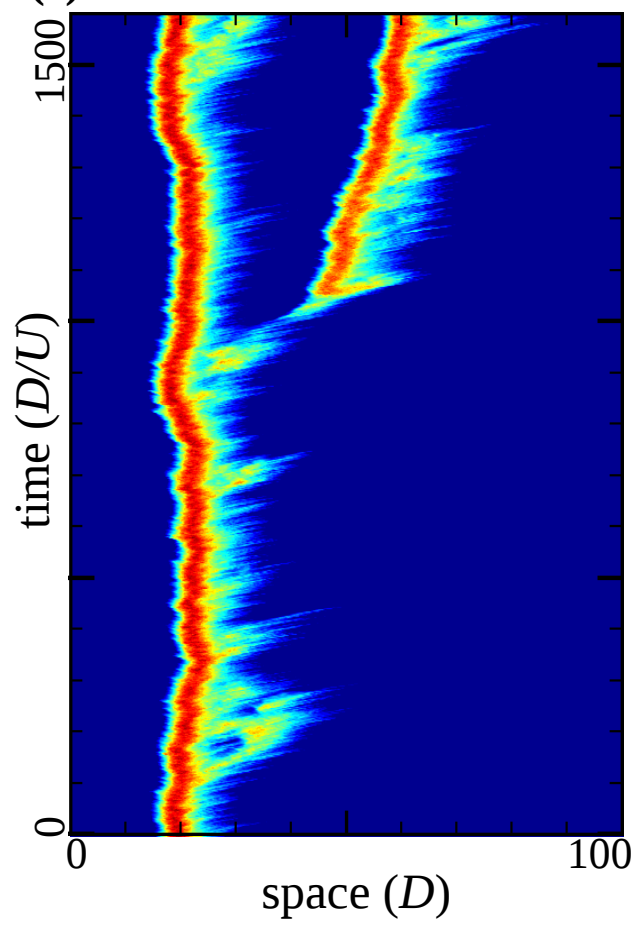

(d)
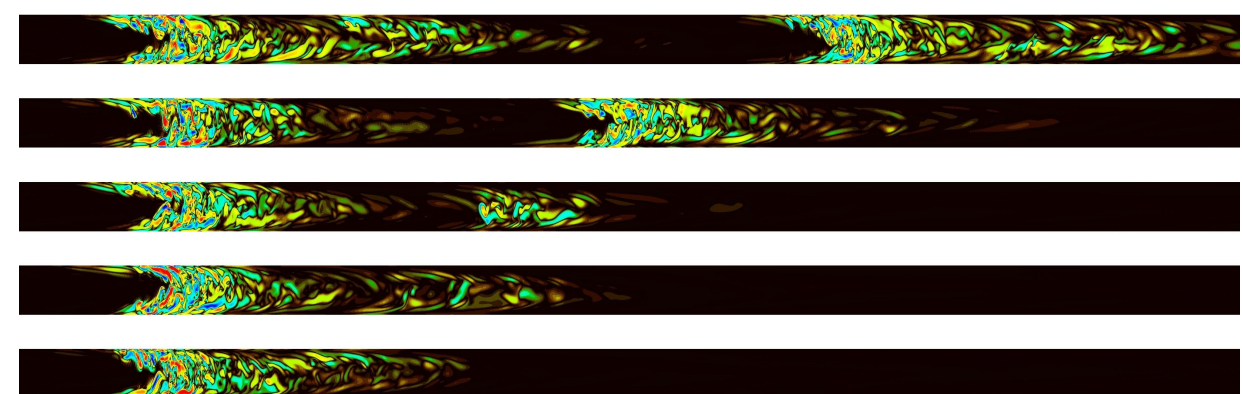

Figure 2.1: Puff splitting in experiment and numerical simulation. (a), (b) Pressure signals from experiment are used to distinguish the case of (a) a split puff from (b) a single puff. A splitting is registered if the signal has peaks separated by $20 D$ or more and if between peaks the signal drops by at least $30 \%$. The flow between the two puffs does not recover to the fully developed laminar profile. (c) Space-time diagram from numerical simulation using the spectral-element Fourier code at $R e=2350$ showing the splitting process. Streamwise vorticity at the centerline is plotted on a linear scale in a comoving reference frame (speed $U_{p}=0.912 U$ ). The upstream edge of a puff is relatively well defined while the downstream edge is fuzzy and fluctuates. Following a splitting the two puffs propagate downstream, separated by an approximately constant distance, and generate a twin-peaked pressure signal (a). (d) Visualization of puff splitting in a cross-sectional $(x, y)$-plane, with red (blue) as positive (negative) streamwise vorticity on a linear scale in a frame moving with the trailing edge velocity. $66 \mathrm{D}$ of a $88 \mathrm{D}$ simulation domain is shown. At $t=0$ (bottom panel) $R e$ is impulsively changed from 2200 to 2250 . Snaphots are taken at $t=0,1436,1537,1575$ and 1612. Once the puff extends far enough and the vorticity decays in its central section, a new puff emerges. 


\subsection{Introduction}

The seemingly simple question as to when the flow down an ordinary pipe turns turbulent dates back to the pioneering study of Osborne Reynolds in the late 19th century (Reynolds 1883). Reynolds proposed that below a critical velocity pipe flows are always laminar, whereas above turbulence prevails given the right initial conditions. The observation that this critical point can be expressed in a dimensionless form was the basis of one of the central concepts in fluid dynamics: the Reynolds number $(\operatorname{Re}=U D / v$, where $U$ is the mean velocity, $D$ the pipe diameter and $v$ the kinematic viscosity). Curiously, while the Reynolds similarity has proved to be valid throughout fluid mechanics, the value of the critical point in pipe flow has been debated ever since. Values reported in textbooks and journal papers vary widely, typically ranging from 1700 to 3000 (Darbyshire \& Mullin 1995, Eckhardt 2008, 2009).

One circumstance that complicates this problem is that laminar pipe flow is stable to infinitesimal perturbations (Drazin \& Reid 2004, Meseguer \& Trefethen 2003) and therefore in order to trigger turbulence a disturbance of finite amplitude is required (Reynolds 1883). What makes matters even more difficult is that at low Re turbulence is transient. Here turbulence occurs in the form of localized patches called 'puffs' (Wygnanski \& Champagne 1973) which are embedded in the surrounding laminar flow and decay according to a memoryless process (Eckhardt et al. 2007). The rapid increase in lifetime with $R e$ has led to various proposed values for a critical point where the lifetime would diverge and turbulence would become sustained (Faisst \& Eckhardt 2004, Willis \& Kerswell 2007, Peixinho \& Mullin 2006). However, more detailed studies (Hof et al. 2006, 2008, de Lozar \& Hof 2009, Avila et al. 2010, Kuik et al. 2010) have shown that the lifetime of individual puffs remains finite and only approaches infinity in the infinite $R e$ limit. In analogy to observations in model systems known as coupled-map lattices (Kaneko 1985), the point has been raised that in addition to purely temporal aspects, spatial dynamics are important for the long term behavior in fluid systems (Pomeau 1986, Bottin \& Chaté 1998, Bottin et al. 1998, Manneville 2009). Spatial coupling can give rise to a critical point where complex dynamics is sustained even though individual turbulent patches are transient. In a numerical study of pipe flow, Moxey and Barkley (Moxey \& Barkley 2010) observed that at $R e \approx 2300$ turbulent puffs delocalize and the turbulent fraction increases, as expected in a system with spatio-temporal dynamics. However, the stochastic nature of the spatial coupling was not taken into account and the extremely long timescales intrinsic to the flow could not be resolved in the simulations. In this work we resolve the critical point in pipe flow and quantify the relevant process sustaining turbulence in this and other linearly stable shear flows.

\subsection{Long-pipe experiments}

Determining the point where the proliferation of turbulence outweighs its decay and turbulence eventually becomes sustained requires that the timescales of both decay and spreading processes be captured. Since turbulent puffs move downstream at approximately the mean flow velocity, a long pipe is required to observe long timescales. Using a precision glass tube with a relatively small diameter $(D=4 \pm 0.01 \mathrm{~mm})$ and overall length 
of $15 \mathrm{~m}$, a total dimensionless length of $3750 \mathrm{D}$ is achieved. The pipe is composed of 14 sections joined by machined perspex connectors which provide an accurate fit. A smooth inlet together with careful alignment of the individual pipe sections allows the flow to remain laminar up to $R e=4400$. Deviations in $R e$ were kept below \pm 5 throughout each set of measurements, which extended over periods of up to $45 \mathrm{~h}$. This precision was achieved by stringent control of both the pressure difference driving the flow and the fluid (water) temperature $( \pm 0.05 \mathrm{~K})$. A detailed description of the experimental setup can be found in (Hof et al. 2008).

Starting from a fully developed laminar flow allows us to induce turbulence in a controlled manner and quantify the spreading rate at some downstream position. The experimental procedure is to create a single turbulent puff close to the pipe inlet and to monitor any changes in the turbulent fraction at downstream positions. It is important that a perturbation is chosen which efficiently triggers turbulence. In many earlier studies, e.g. (Wygnanski \& Champagne 1973, Rotta 1956), turbulence was induced by insertion of a static obstacle close to the pipe inlet. Such obstacles provide a continuous perturbation and at high Reynolds numbers the flow downstream is fully turbulent whereas in the transitional regime $(R e \lesssim 3000)$ irregular sequences of turbulent and laminar phases are created (Rotta 1956). At lower Reynolds numbers $(R e \approx 2000)$ on the other hand sufficiently strong continuous perturbations cause plug shaped velocity profiles which are unable to sustain turbulence (Hof et al. 2010) and lead to relaminarization. Hence even for large disturbance levels continuous perturbations will fail to trigger turbulence at low Reynolds numbers. To avoid such problems in the present study an impulsive perturbation was chosen, consisting of a water jet injected through a circular hole of $0.2 D$ in the pipe wall $250 D$ downstream of the inlet. The duration of the perturbation was adjusted for each set of measurements $(8-20 \mathrm{~ms}$, corresponding to advection of approximately $1-2.5 \mathrm{D}$ at the mean flow velocity) ensuring that only one single puff is generated from each perturbation. Different amplitudes were tested and the results were found to be independent of the perturbation strength. In relation to the mass flow in the pipe, typical injection rates were about $2.5 \%$. To establish that results are independent of the perturbation, additional measurements were carried out using an obstacle to impulsively disturb the flow. A thin wire $(0.8 \mathrm{~mm}$ in diameter and $10 \mathrm{~mm}$ in length) was inserted into the pipe $150 \mathrm{D}$ from the inlet. The wire was held against the pipe wall and aligned with the pipe axis using a small magnet attached to the outside of the pipe. When against the wall the disturbance created by the wire is too small to trigger turbulence for the $R e$ investigated. When the wire was moved impulsively, (azimuthally along the pipe wall by approximately $0.5 \mathrm{~mm}$ over about $0.1 \mathrm{~s})$, a single turbulent puff was triggered.

After triggering disturbances, the flow is monitored by two downstream pressure sensors (Fig. 2.6). The first one, located $300 \mathrm{D}$ from the inlet, confirms that each perturbation results in the creation of a single puff. The second one, which can be positioned at various distances $L$ from the perturbation, is used to distinguish cases where multiple puffs arrive (Fig. 2.1(a)) from those where only the single puff arrives (Fig. 2.1(b)). This is a direct measurement of whether the turbulent fraction in the flow has increased or remained constant during downstream propagation. 
(a)

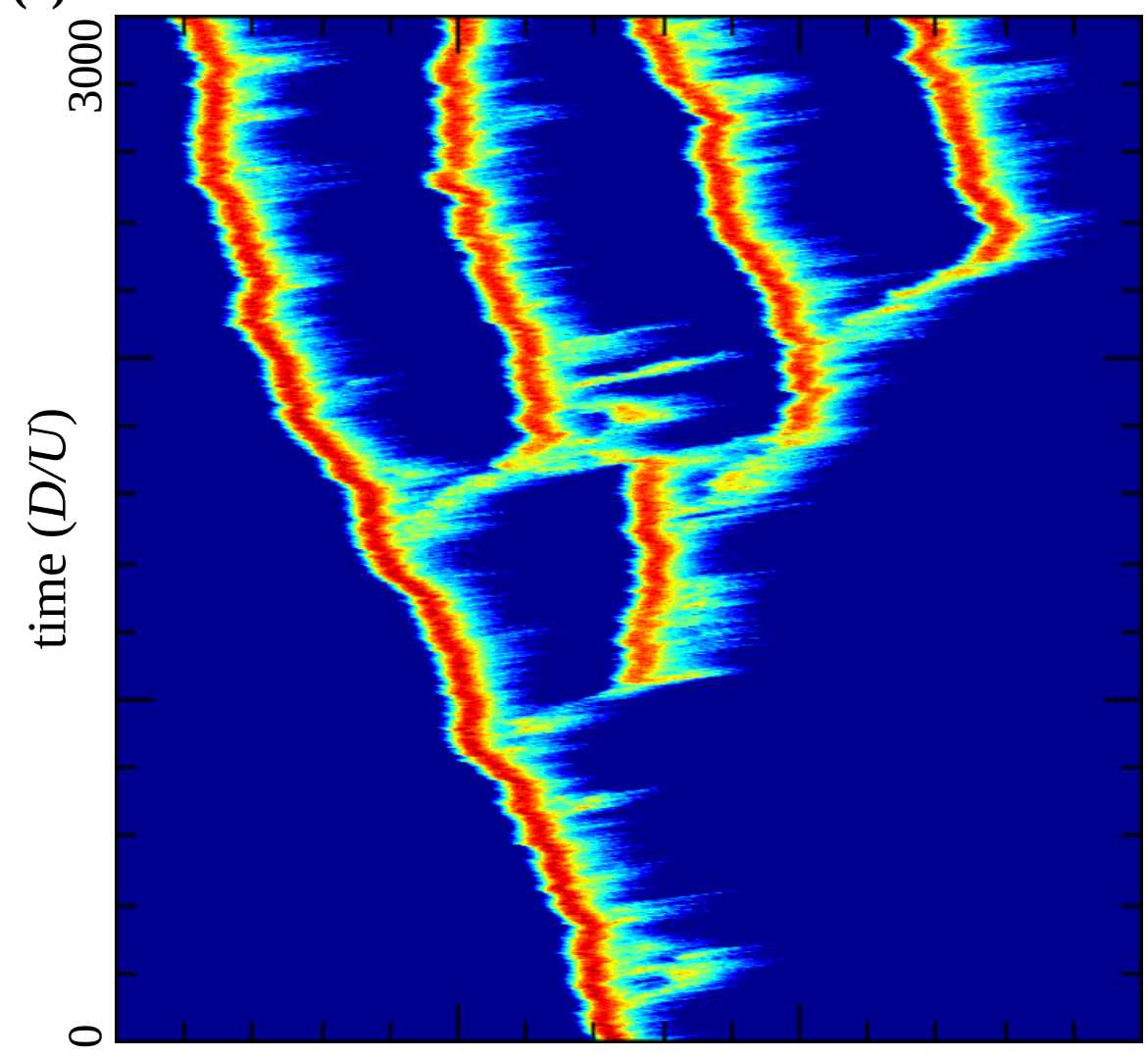

(b)

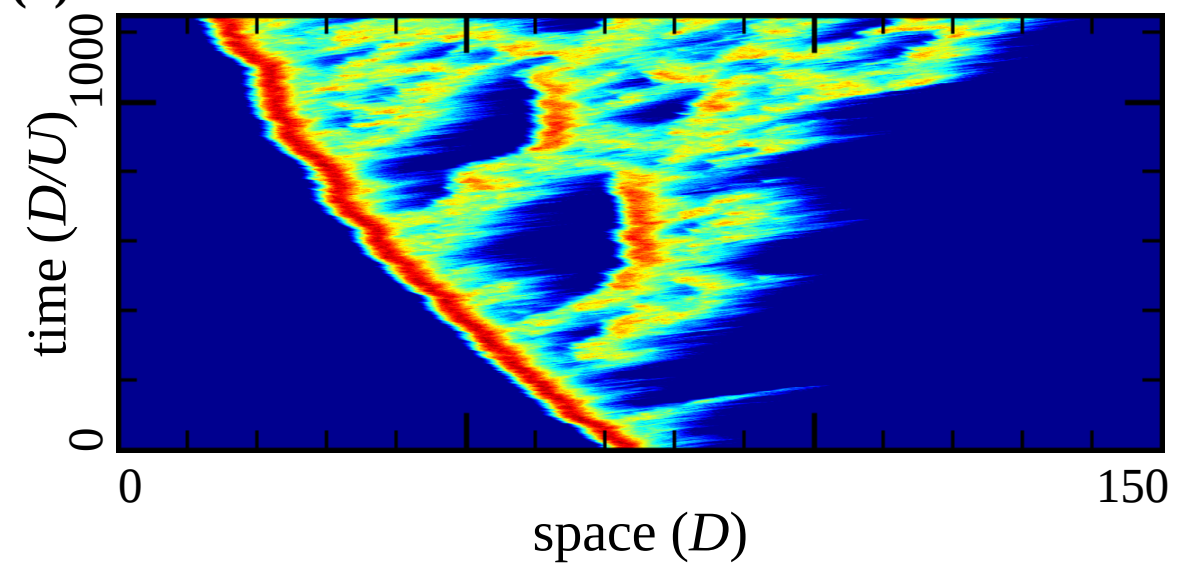

Figure 2.2: Spreading of turbulence in numerical simulation. Space-time diagram at $R e=2300$ (a) and 2450 (b) from numerical simulation using the hybrid spectral finitedifference code showing how turbulence proliferates starting from a localized puff at $R e=$ 2200 as initial condition. The cross-sectional average of streamwise vorticity squared is plotted on a logarithmic scale in a co-moving reference frame at speed $0.947 U$ and $0.94 U$, respectively. At $R e=2300$ the expansion process is dominated by discrete steps, corresponding to puff splits, while at 2450 expansion is more smooth, more rapid, and individual puffs are no longer easily identified. 


\subsection{Simulations}

To complement experiments and gain insights into the underlying spread of turbulence, we have carried out extensive numerical simulations. Two independent numerical codes have been used; one is a spectral-element Fourier code (Blackburn \& Sherwin 2004) which solves the Navier-Stokes equations in Cartesian coordinates (DNS1), and the other a hybrid spectral finite-difference code (Willis \& Kerswell 2009) which solves the equations in cylindrical coordinates (DNS2). Both methods use periodic boundary conditions in the streamwise direction and impose constant unit mass flux, ensuring no variation in $R e$ during any run. The codes have been tested both against each other, producing statistically identical results, and by increasing the resolution of the discretizations. Details have been reported elsewhere (Avila et al. 2010, Moxey \& Barkley 2010) -(see also supporting online materials).

\subsection{Puff splitting}

For all of the lower $R e$ under investigation, an increase in the turbulent fraction manifests itself in the form of 'puff splitting' (Wygnanski \& Champagne 1973, Nishi et al. 2008), where new puffs are seeded downstream of existing ones (Fig. 2.1(c) and (d)). Directly downstream of a turbulent puff the flow has not recovered a parabolic profile and cannot sustain turbulence (Hof et al. 2010). To successfully seed a new puff, a patch of vorticity has to escape far enough downstream from the initial puff to an area where the velocity profile is sufficiently parabolic. This process can be observed in Fig. 2.1(c) where regions of large vorticity fluctuations repeatedly propagate downstream and decay. Only during a sufficiently large excursion does a new puff arise downstream of the old one.

Fig. 2.2(a) illustrates how, starting from a single puff, turbulence proliferates through a sequence of splittings at $R e=2300$. For $R e \leq 2300$ for all our measurements (numerical as well as experimental) spreading of turbulence and hence any increase in turbulence fraction (Fig. 2.10) was exclusively observed in the form of puff splitting. Only at Reynolds numbers somewhat larger than considered here $(\operatorname{Re}>2400)$ do individual puffs start to noticeably expand (Fig. 2.2(b)) such that the overall spreading of turbulence becomes a complex mixture of splitting as well as growth and merging of individual turbulent domains.

\subsection{Characteristic time of memoryless process}

Due to the stochastic nature of the splitting process, a statistical approach must be used. To investigate splitting statistics, we have performed ensembles of numerical simulations starting from independent puffs (Table 2.3) from which we determine the time for each to split. As shown in Fig. 2.3, the probability for a puff to remain localized, i.e. not split, can be described by $1-P(R e, t)=\exp \left(-\left(t-t_{0}\right) / \tau(R e)\right)$ where $t$ is the observation time, $t_{0}$ is a formation time and $\tau(R e)$ is the $R e$ dependent characteristic time for the process. The formation time $t_{0}$ includes any equilibration time for the initial condition to evolve to the turbulence attractor at the particular $R e$ and the intrinsic time needed for splitting. In principle $t_{0}$ depends on $R e$ as well as on the initial conditions used in ensemble runs, but 


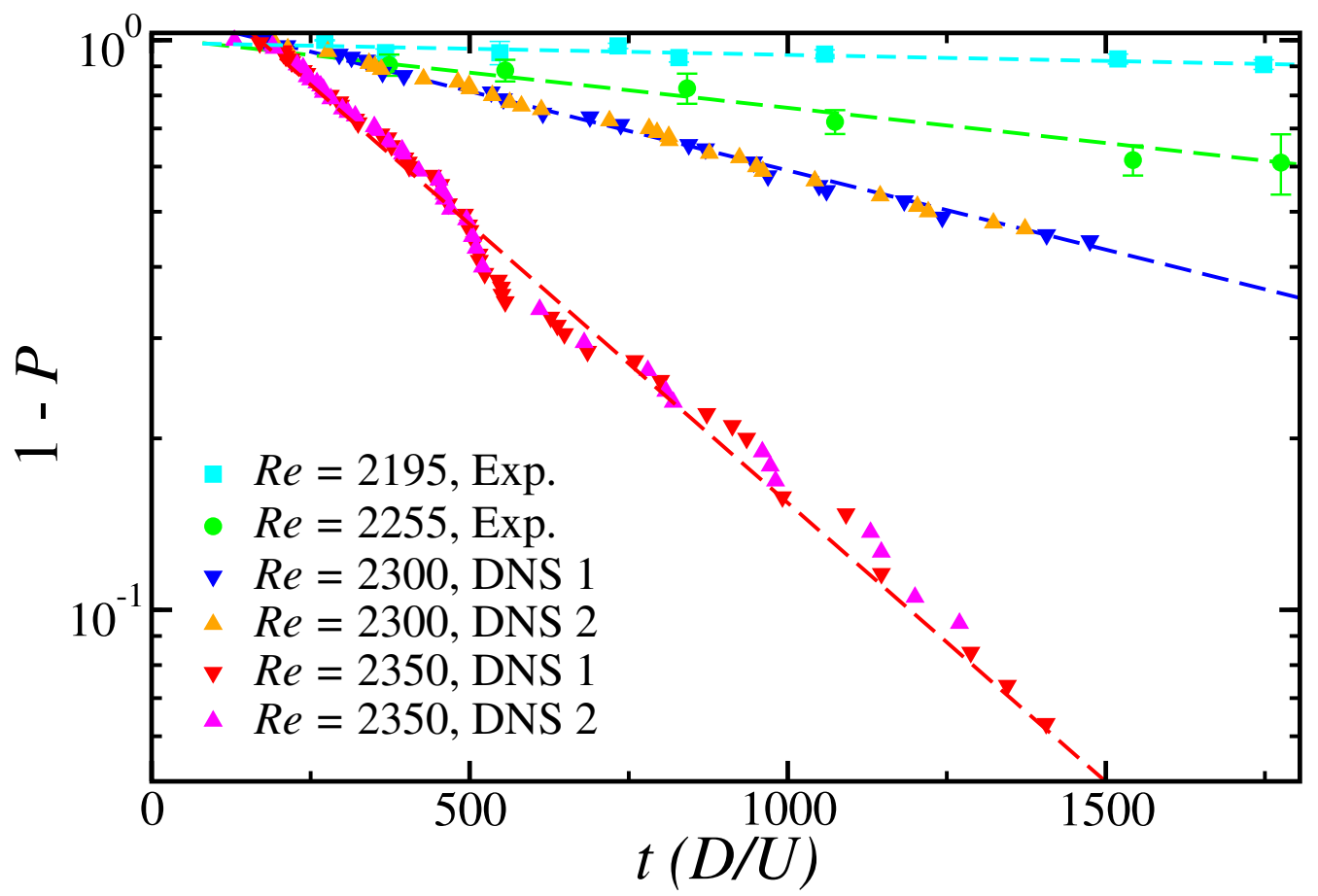

Figure 2.3: Probability distributions for a puff to remain in equilibrium. $P$ is the probability that a puff will split before time $t$. Hence the plotted quantity $1-P$ is the probability that a puff remains a single localized puff up to time $t$. The numerical distributions at $R e=2300$ and $R e=2350$ are obtained from all first splitting times in ensembles of simulations using both the spectral-element Fourier code (DNS 1) and the hybrid spectral finite-difference code (DNS 2). Experimental distributions at $R e=2195$ and 2255 are obtained from statistics collected from fixed downstream locations $L$ converted to time by the $R e$ dependent mean puff propagation speed $U_{p}$. All distributions are of the form $\exp \left(-\left(t-t_{0}\right) / \tau\right)$, as illustrated by the dashed lines, where $t_{0}$ is a development time for splitting to take place $\left(t_{0} \approx 150\right.$ for DNS, while for experiment $t_{0}$ is nearer to 100 and has greater uncertainty.) The exponential form of the distributions indicates that splitting is a memoryless process with characteristic time $\tau$.

from the two detailed cases in Fig. 2.3 as well as from the time of first splitting at other $R e$, we find consistently $100 \lesssim t_{0} \lesssim 200$.

Experimental measurements (Fig. 2.3 circels and squares) also reveal exponential distributions. Observations were carried out at different distances, $L$, which are translated into observation time by $t=L / U_{p}$, where $U_{p}=1.482-2.416 \times 10^{-4} R e$ is an approximation to the mean puff speed in this $R e$ range, as obtained by numerical simulation (see Fig. 2.6).

Beyond the initial formation time the distributions are exponential and hence memoryless, reflecting that the probability of splitting does not depend on the age of the puff under investigation. The splitting probability is constant in time and characterized solely by $\tau$, which after the initial formation time gives the mean time for a puff to split and the turbulence fraction to increase.

Experimentally it is far easier to keep the observation point fixed during one series 


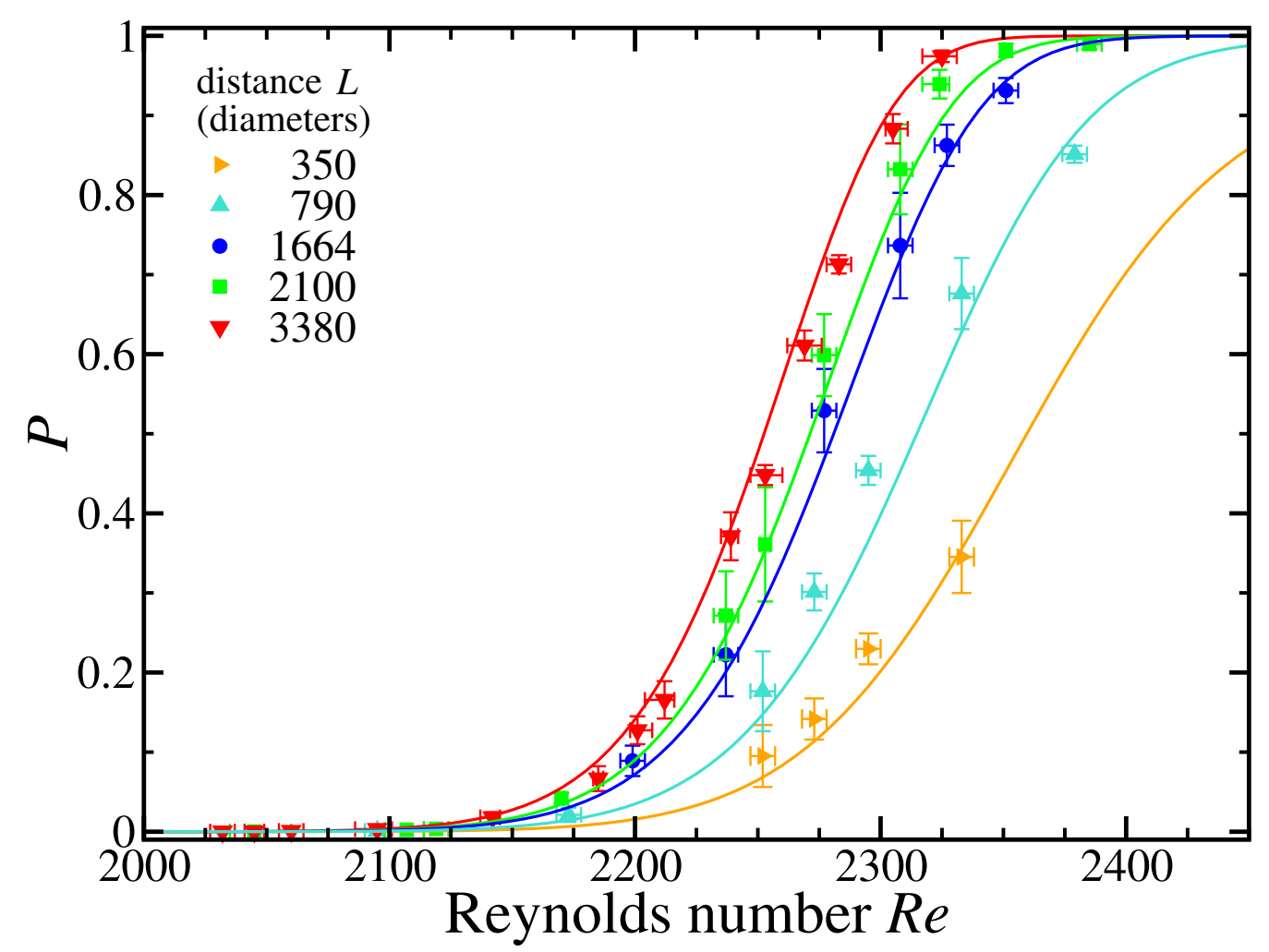

Figure 2.4: Probability of puff splitting after traveling a fixed distance. The five data sets correspond to distances $L$ in the experiments as indicated in the legend. Here the splitting probability $P$ is computed as $P=k / n$, where $k$ is the number of events that split and $n$ the total number of realizations. The error bars in the vertical direction are $95 \%$ confidence intervals for the parameter $P$ of a binomial distribution as a function of $(n, k)$ obtained with the Wilson method (Brown et al. 2001), whereas in the horizontal direction they show the uncertainty in $R e$ during a set of measurements. The solid lines correspond to the super-exponential fit from Figure 2.5 without additional fitting parameters.

of measurement and vary $R e$ in order to determine $P(R e, t)$ from which $\tau(R e)$ can be obtained. The results for five distances $L$ are shown in Fig. 2.4. For each data point typically 2000 measurements are performed, but for the lowest $R e$, up to 60,000 measurements are used. As expected, at large $R e$ the splitting probability is high and decreases as $R e$ is reduced. Curves for fixed $L$ are $S$-shaped indicating that $P \rightarrow 0$ only asymptotically as $R e$ decreases. All measurements are well approximated by a single super-exponential fit with only two-parameters, $\tau=\exp (\exp (a R e+b))$, where $a=-0.003115$ and $b=9.161$ (solid lines in Fig. 2.4). In contrast to earlier studies (Moxey \& Barkley 2010, Wygnanski \& Champagne 1973, Nishi et al. 2008) our data indicate that there is no critical point where the spatial proliferation of turbulence abruptly sets in. The tendency to split appears to be intrinsic to turbulence even at low Reynolds numbers and turbulent patches are not in an equilibrium state (Wygnanski et al. 1975). It is hence the stochastic details of this process that decide if turbulence will either invade the entire domain or recede and eventually decay. 


\subsection{Critical point}

To determine this critical point for the onset of sustained turbulence it is necessary to compare the timescale for turbulence to spread through splitting to the timescale for turbulence to decay. The dependence of the mean splitting time on $R e$ is plotted in Fig. 2.5. From each experimental data point in Fig. 2.4 the mean time $\tau$ is obtained and plotted (colored symbols on right branch in Fig. 2.5), together with the single super-exponential fit (solid line in Fig. 2.5). In order to obtain $\tau$ from the experimental probabilities a formation time of $t_{0}=100$ and uncertainties in the splitting time are included in the error bars. Note that because in experiments observation times are generally large the uncertainties in $t_{0}$ are negligible compared to statistical errors.

Additionally, $\tau$ values from simulations are included in Fig. 2.5 (black solid triangles), showing that results from both numerical codes are in excellent agreement with the experimental data. From ensemble simulations one obtains directly the times for each split and one obtains a maximum likelihood estimate of $\tau$ from the memoryless character of the splitting process (Avila et al. 2010, Lawless 2003). The rapid-increase in splitting times make it infeasible to obtain $\tau$ numerically at low $R e$.

The left branch in Fig. 2.5 summarizes previously measured mean lifetimes for turbulent decay (Hof et al. 2008, Avila et al. 2010, Kuik et al. 2010) together with a single super-exponential fit for mean lifetimes. The intersection at $R e \approx 2040$ marks where the mean lifetime is equal to the mean splitting time and to the right of the intersection splittings outweigh the decay of puffs. Analyzing the data in terms of the turbulent fraction results in the same critical point (see Fig. 2.10) confirming the procedure applied here.

Typically in statistical phase transitions, critical points are not identical to the exact balance point of two competing processes due to correlations. For example in the standard contact process (Harris 1974, Hinrichsen 2000a) the spreading (contamination) rate of an active phase has to outweigh its decay (recovery) rate by a ratio of about 3 before the active phase becomes sustained. While in the present case the long timescales make it impossible to measure other signatures of criticality such as scale invariance, the superexponential scaling of the two processes ensures that the critical point will be almost indistinguishable from the intersection point. For an increase in $R e$ of 10 (or $0.5 \%$ ) above the critical point, the splitting rate already outweighs the decay rate by a factor of 4 . Therefore the difference between the intersection point and the critical point is of the same order as the experimental uncertainty in $R e$ and $2040 \pm 10$ provides a close estimate of the critical point for the onset of sustained turbulence. 


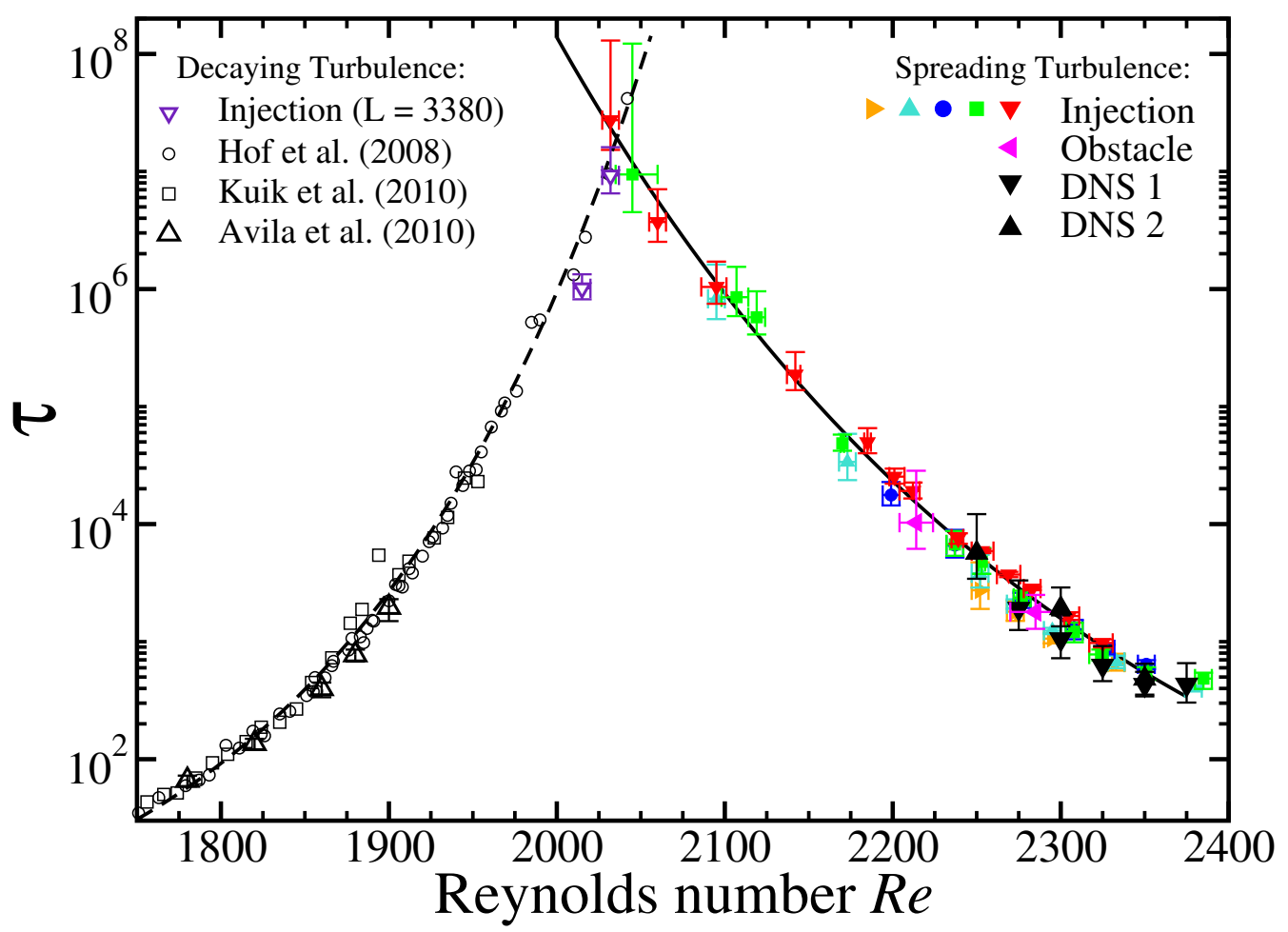

Figure 2.5: Mean lifetime of a puff before decaying or splitting. Full colored symbols correspond to experimental splitting measurements. Measurements downstream of jet injection are at different distances $L$ as indicated in Fig. 2.4. Measurements downstream of an obstacle perturbation are at $L=1700$. The full black triangles show the characteristic splitting time obtained from direct numerical simulations using the spectral-element Fourier code (DNS 1) and the hybrid spectral finite-difference code (DNS 2). The solid curve given by $\tau=\exp (\exp (-0.003115 R e+9.161))$ and approximates the Reynolds number dependence of mean time until a second puff is nucleated and the turbulent fraction increases. This curve was obtained by fitting the data sets with distances $L=1664,2100$ and 3380, whereas results from shorter pipes were not fitted due to the stronger influence of the uncertainty in $t_{0}$. The dashed curve is given by $\tau=\exp (\exp (0.00556 R e-8.4988))$ and approximates the mean time until turbulence decays and the flow relaminarizes. Both curves correspond to super-exponential scaling with $R e$ and have a crossover at $R e=2040 \pm 10$, determining the transition between transient and sustained turbulence in pipe flow in the thermodynamic limit. 


\subsection{Conclusion}

The complexity of the transition process encountered in pipe flow is common to many shear flows, including Couette, channel, duct and boundary layer flows. In all these cases turbulence is found despite the stability of the base flow and first takes the form of localized patches which are transient. The key to the approach here to determine the onset and sustainment of turbulence has been to separate the analysis of decay and proliferation mechanisms, and this approach should be equally applicable even though details of these mechanisms may differ from case to case. The intermittently alternating laminar and turbulent regions encountered in pipe flow just above criticality are intrinsic to the problem and place pipe flow in the larger theoretical framework of spatiotemporal intermittency (Kaneko 1985, Chaté \& Manneville 1988) and nonequilibrium phase transitions where universal scaling properties may be expected (Rolf et al. 1998, Hinrichsen 2000a). While in the present study the spatial interaction is relatively simple due to the clear separation of adjacent puffs, further above the critical point $(R e \gtrsim 2400)$ the dynamics quickly becomes increasingly complicated with domains merging and annihilating. To comprehend this increasing spatial complexity sets challenges for future studies and is key to our understanding of the onset and nature of turbulence.

\subsection{Acknowledgments}

We thank A. P. Willis for sharing his hybrid spectral finite-difference code. We acknowledge the Deutsche Forschungsgemeinschaft (project FOR 1182), the Max Planck Society for financial support. DB thanks the Leverhulme Trust and the Royal Society, and the Engineering and Physical Sciences Research Council(grant EP/F017413/2) for financial support. D.B. thanks the Leverhulme Trust and the Royal Society for their support. MA and $\mathrm{BH}$ acknowledge computing resources from GWDG and the Jülich Supercomputing Centre (grant HGU16), where DNS2 were performed. DM and DB acknowledge computing resources from the Centre for Scientific Computing, University of Warwick and Grand Equipement National de Calcul Intensif-Institut du Développement et des Ressources en Informatique Scientifique (grants 2010-1119 and 2011-1119) where DNS1 were performed. K.A. acknowledges support from the International Max Planck Research School for the Physics of Biological and Complex Systems and the Göttinger Graduate School for Neurosciences and Molecular Biosciences. 


\subsection{Supplementary Online Material}

\subsubsection{Materials and Methods}

Numerical Simulations

The numerical results presented here have been obtained with two distinct methods. The first (DNS1) is the spectral-element-Fourier code Semtex (Blackburn \& Sherwin 2004), which solves the Navier-Stokes equations in Cartesian coordinates $(x, y, z)$. 36 spectral elements are used to represent the circular $(y, z)$ cross-sections of a pipe. Elements are placed to mimic the radial distribution of Chebyshev collocation points, with fields approximated using a 12th order polynomial expansion basis within each element. Fourier modes are used in the periodic axial direction at a density of 768 complex modes (1536 collocation points) per $100 D$ in pipe length.

The second (DNS2) is the hybrid spectral finite-difference method of Willis \& Kerswell (2009), which solves the equations in cylindrical coordinates $(x, r, \theta)$. The numerical discretization consists of a non-equispaced 9-point finite-difference stencil in $r$ and of Fourier modes in $\theta$ and $z$. Here 56 radial points, \pm 32 azimuthal Fourier modes and \pm 1024 axial Fourier modes (64 and 2048 collocation points, respectively) have been used per $L=32 \pi D \simeq 100 D$ in pipe length.

Both methods use periodic boundary conditions in the streamwise direction, fix the diameter at $D=1$, and impose constant volume flux (so that $U=1$ ), ensuring no variation in $R e$ (which is given by $1 / v$ ), during any run.

Initial conditions for ensemble runs where obtained from snapshots of simulation of puffs at lower $R e$, similarly to the approach in puff lifetime studies (Avila et al. 2010). In the case of the spectral-element-Fourier (DNS1) runs, all such initial conditions were generated from simulations at $R e=2100$ with snapshots taken every $20(D / U)$. In the case of the hybrid spectral finite-difference (DNS2) runs, all such initial conditions were generated from simulations at $R e=2200$ with snapshots taken every $10(D / U)$.

Experiments: Pressure measurements

Pressure measurements are performed at two positions along the pipe. The first measurement is made $50 \mathrm{D}$ downstream of the perturbation in order to verify the generation of a single puff by the perturbation. The second one is located at various distances $L>50 D$ from the perturbation in order to determine the subsequent turbulent fraction. Sensors measure the pressure difference over $3 D$ along the pipe. Pressure sensors are connected via tubes to pressure taps in the pipe wall as illustrated in Fig. 2.6. We used the low pressure sensor DP45 from Validyne with the diaphragm no. 14 (0.021 - 0.049 PSI). Special care was taken to remove all airbubbles from the tubes and sensor prior to measurements. Such air pockets could alter the signal and even reinduce disturbances into the pipe under unsteady flow conditions.

Puff speed

To convert between distances and times and to plot puffs in co-moving frames of reference, We use the following approximation for the Reynolds number dependent puff speed:

$$
U_{p}=1.482-2.416 \times 10^{-4} R e
$$

This is shown graphically in Fig. 2.7. 
(a)

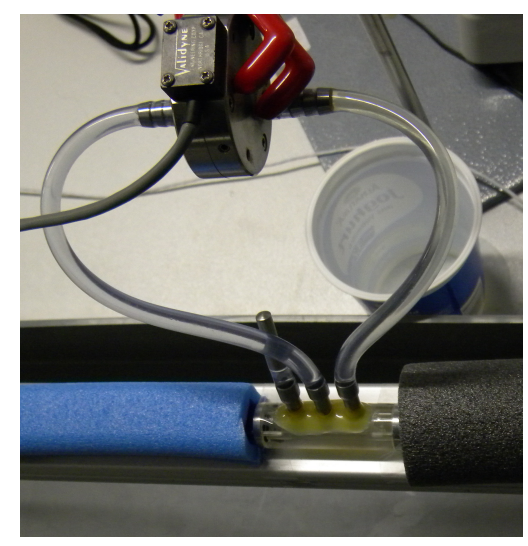

(b)

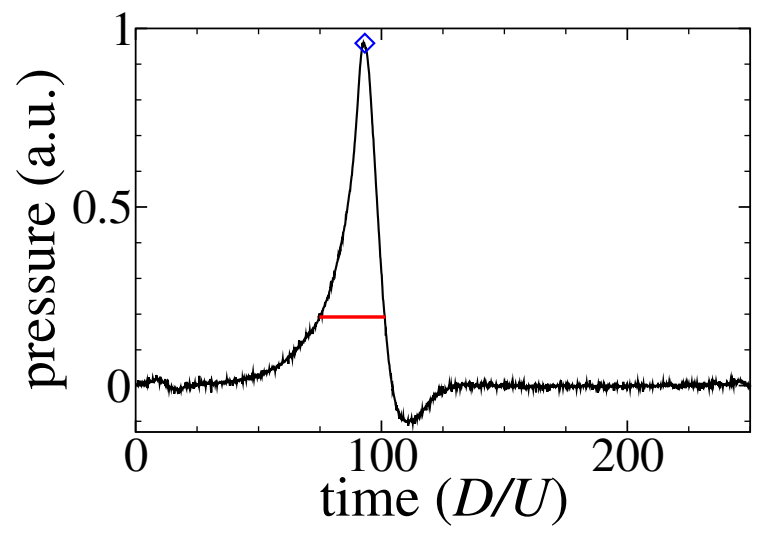

Figure 2.6: Pressure difference measurement at a machined perspex connector. (a) The connector features three holes of $0.2 D$ diameter and separated by $3 D$, of which here two neighboring ones are used for the pressure measurement. Air bubbles are removed from the sensor and the tubing prior to usage. The precision glass tube to the left and right are covered by insulating materials to minimize temperature effects and light exposure (to avoid algae growth). (b) The pressure signals are then analyzed to detect the number of peaks (here one, marked by the blue diamond) and the length of the turbulent region at $20 \%$ of the maximal pressure peak, indicated by the red line.

Obtaining confidence intervals for DNS

As seen in Table 2.3, each observed lifetime $t_{1}, \ldots, t_{n}$ obtained through DNS is censored by imposing an upper bound on the simulation time so that $t_{i} \leq t_{\max }$. In Lawless (2003) this is defined as type I censoring, for which exact confidence intervals do not exist and must therefore be approximated numerically. Given the small number of samples, the central limit theorem provides inaccurate intervals, and so here we opt to use the technique of bootstrapping as seen in appendix D.2 of Lawless (2003). The procedure for generating confidence intervals is as follows:

1. Generate a pseudorandom sample $t_{1}^{*}, t_{2}^{*}, \ldots, t_{n}^{*}$ by sampling from the set of lifetimes with replacement.

2. Calculate the maximum likelihood estimator $\hat{\tau}_{1}^{*}$ of this data as

$$
\hat{\tau}_{1}^{*}=\frac{1}{r_{1}^{*}} \sum_{k=1}^{n} t_{k}^{*},
$$

where $r_{1}^{*}$ is the number of uncensored lifetimes and $t_{0}+t_{k}^{*}=t_{\max }$.

3. Repeat the previous two steps $B$ times to obtain bootstrap samples $\left\{\hat{\tau}_{1}^{*}, \hat{\tau}_{2}^{*}, \ldots, \hat{\tau}_{B}^{*}\right\}$.

4. Sort this data so that $\hat{\tau}_{b}^{*} \leq \hat{\tau}_{b+1}^{*}$.

Assuming $B=10^{k}$ where $k \geq 2$, confidence intervals at level $1-\alpha$ may then be read off as $\left[\hat{\tau}_{B \alpha / 2}^{*}, \hat{\tau}_{B(1-\alpha / 2)}^{*}\right]$. 
Analysis of the experimental data

The analysis of each set of experimental measurements leads to a splitting probability $P(\operatorname{Re}, L)=r / n$. Here $L$ is the downstream distance from the perturbation and $r$ is the number of runs that splitted out of a total of $n$ (see Table 2.1). In the first step $L$ is converted into time $t$ by using the puff speed approximation of equation (2.1). The mean time $\tau$ of the splitting process with formation time $t_{0}=100$ is then calculated as:

$$
\tau=\frac{t_{0}-t}{\log (1-P(\operatorname{Re}, t))}
$$

The $95 \%$ confidential intervals $P$ (shown in Fig. 2.4 of the paper) are estimated by the Wilson method (Brown et al. 2001):

$$
P_{\text {error }}=P \pm \frac{1.96 n^{1 / 2}}{\left(n+1.96^{2}\right)}\left(P(1-P)+\left(1.96^{2} /(4 n)\right)\right)^{1 / 2}
$$

By inserting $P_{\text {error }}$ into (2.2) confidence intervals for $\tau$ are obtained. Note that the final error bars for $\tau$ shown in Fig. 2.5 of the paper include the uncertainty in $t_{0} \in[50,150]$. 


\subsubsection{Supporting Figures}

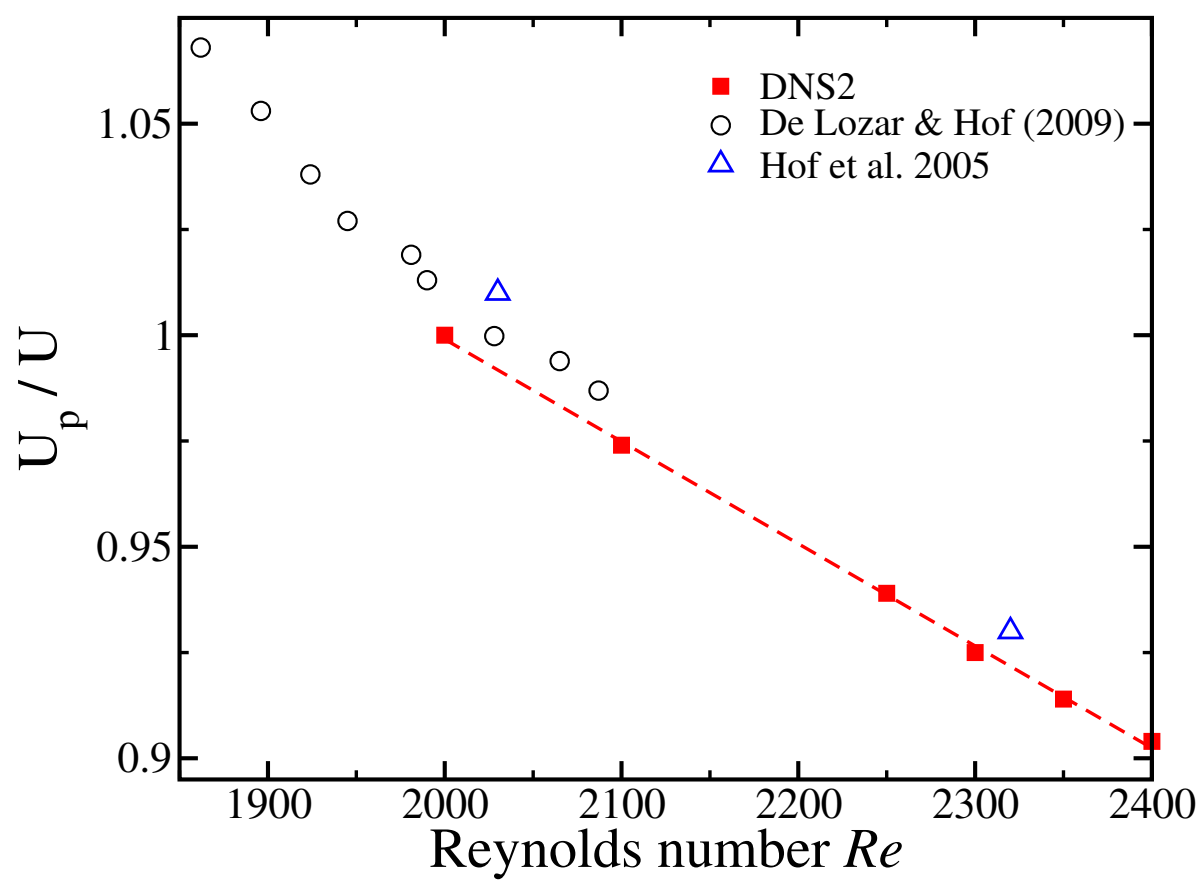

Figure 2.7: Numerically computed puff speed (squares) using the hybrid spectral finitedifference code. The puff speed is very well approximated by Eq. 2.1 over the range $2000 \leq R e \leq 2400$ plotted as red dashed line. Circles are from experiments in de Lozar $\&$ Hof (2009) and triangles from Hof et al. (2005). 


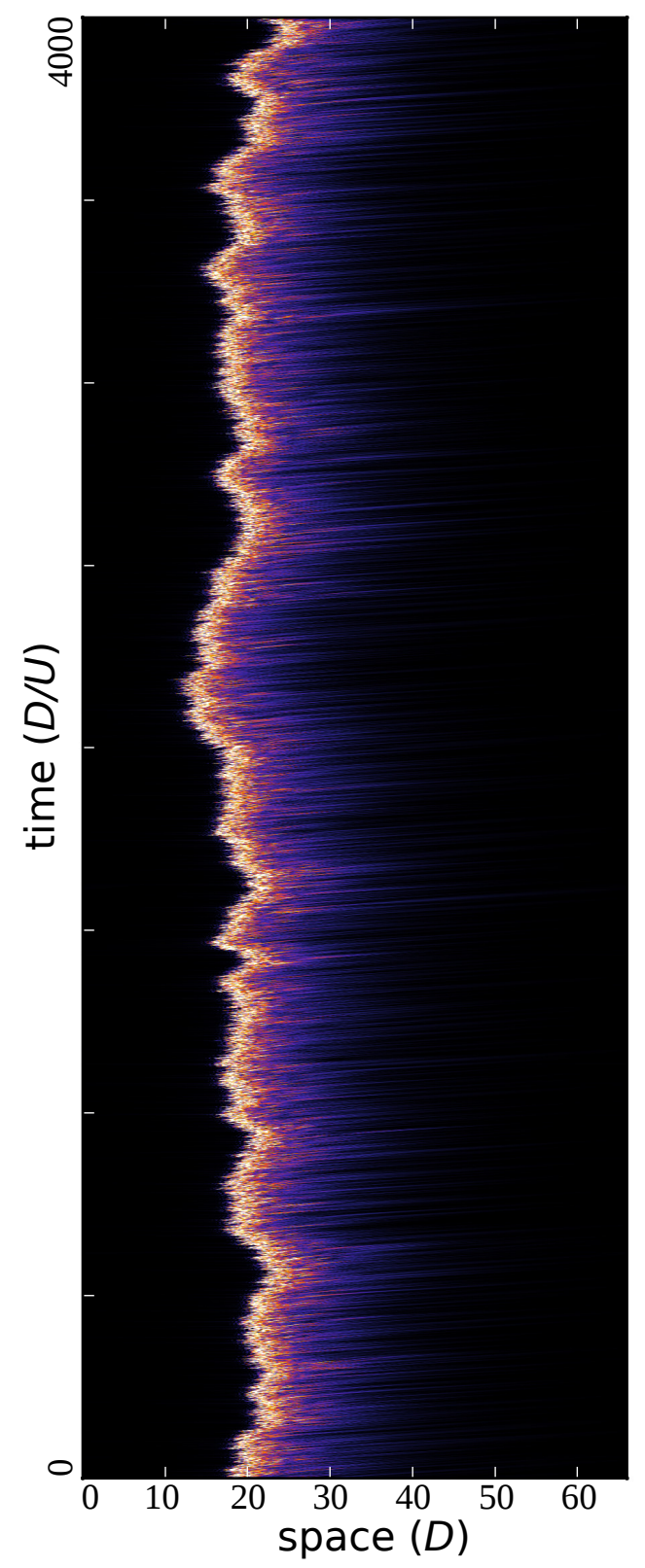

Figure 2.8: Space-time diagram for a puff at the critical Reynolds number $R e=2040$. Streamwise vorticity is plotted on a linear scale in a co-moving reference frame (speed $U_{p}=0.9873 U$ from Eq. 2.1). There are fluctuations in the width and intensity, but on the whole the size and speed of a puff at this $R e$ is constant, i.e. it is an 'equilibrium puff' (Wygnanski et al. 1975). Based on the evidence presented in the paper, the puff will eventually, with nearly equal probability, decay or split. However, the characteristic time for this to occur is more than $10^{7} \mathrm{D} / \mathrm{U}$, considerably longer than the $4 \times 10^{3} \mathrm{D} / U$ shown here. 


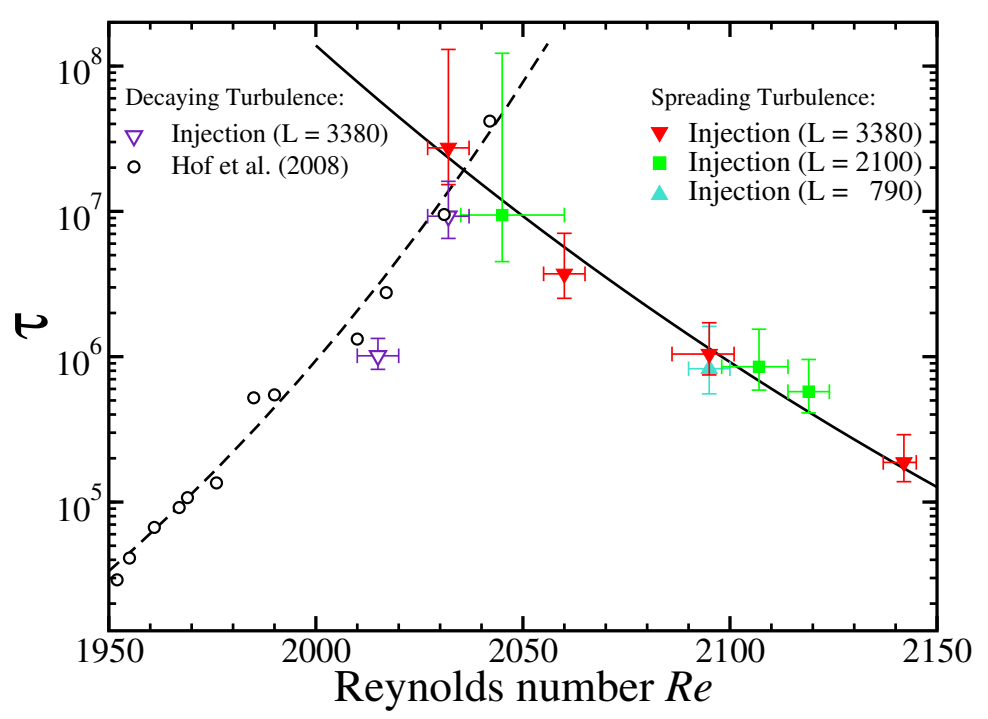

Figure 2.9: Enlargement of Fig. 2.5 in the vicinity of the critical point.

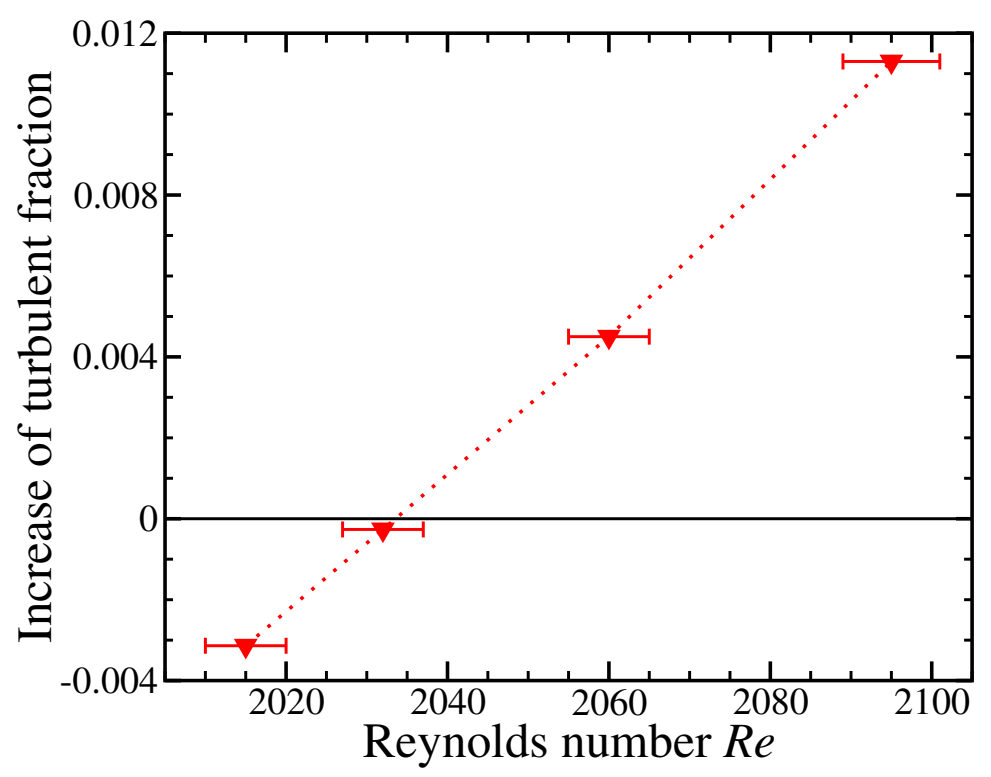

Figure 2.10: Increase of the turbulent fraction from experimental measurements defined as $I=\left(S_{3380}-S_{p u f f}\right) / S_{p u f f}$. At each $R e$ the size of the turbulent region is measured at a distance of $L=3380$ from the perturbation by setting a threshold on the pressure signal (see also Fig. 2.6 (B)). The measurement is subsequently repeated to determine the average turbulent size $\left(S_{3380}\right)$. Finally, the value is normalized with respect to the mean size of a single puff $\left(S_{p u f f}\right)$ measured at this $R e$ using the same threshold. The dotted line connects measurements to guide the eyes. Below the critical point $R e_{c} \simeq 2040$ the turbulent fraction decreases, as indicated by negative values of $I$, whereas for supercritical $R e$ it increases. The error bars in the vertical direction are $95 \%$ confidence intervals of the increase of the turbulent fraction. Note, that they are hidden by the symbol in most of the cases. The size of a single puff is here about $S_{p u f f} \approx 25 D \pm 10 D$. 


\subsubsection{Supporting Table}

Table 2.1: Tabular data for Fig. 2.5 (Experiment) Characteristic splitting time $\tau$ from experiment (jet perturbation) as a function of Reynolds number $R e$ and $t_{0}=100$. Each row corresponds to a set of measurements. The fourth and fifth columns indicate the number of splitting events $r$ and total number of runs $n$.

\begin{tabular}{|c|c|c|c|c|c|c|c|c|c|}
\hline$L$ & $R e$ & $\tau$ & $r$ & $n$ & $L$ & $R e$ & $\tau$ & $r$ & $n$ \\
\hline \multirow[t]{4}{*}{350} & 2252 & 2735 & 21 & 221 & \multirow[t]{12}{*}{3380} & 2032 & 27338718 & 7 & 57823 \\
\hline & 2273 & 1801 & 99 & 699 & & 2060 & 3715436 & 18 & 20073 \\
\hline & 2295 & 1062 & 415 & 1806 & & 2095 & 1042648 & 26 & 8074 \\
\hline & 2333 & 663 & 144 & 417 & & 2142 & 187364 & 31 & 1722 \\
\hline \multirow[t]{8}{*}{790} & \multirow{8}{*}{$\begin{array}{l}2095 \\
2173 \\
2252 \\
2273 \\
2295 \\
2333 \\
2379\end{array}$} & 826518 & 17 & 19844 & & 2185 & 49835 & 66 & 989 \\
\hline & & 33776 & 22 & 1037 & & 2201 & 25358 & 178 & 1397 \\
\hline & & 3817 & 39 & 221 & & 2212 & 19142 & 160 & 966 \\
\hline & & 2080 & 451 & 1497 & & 2239 & 7525 & 366 & 986 \\
\hline & & 1240 & 1302 & 2868 & & 2253 & 5896 & 2666 & 5950 \\
\hline & & 673 & 282 & 417 & & 2269 & 3728 & 1562 & 2557 \\
\hline & & 404 & 3600 & 4229 & & 2283 & 2830 & 4226 & 5928 \\
\hline & & 404 & 5000 & 4229 & & 2305 & 1654 & 1029 & 1165 \\
\hline \multirow[t]{5}{*}{1664} & \multirow{2}{*}{$\begin{array}{l}2199 \\
2237\end{array}$} & \multirow{2}{*}{$\begin{array}{l}17702 \\
6633\end{array}$} & \multirow{2}{*}{$\begin{array}{l}76 \\
54 \\
182\end{array}$} & \multirow{2}{*}{$\begin{array}{l}854 \\
243 \\
344\end{array}$} & & 2325 & 975 & 1825 & 1873 \\
\hline & & & & & \multicolumn{2}{|c|}{ Obstacle } & & & \\
\hline & 2308 & 1274 & 123 & 167 & 1700 & 2214 & 9719 & 8 & 50 \\
\hline & 2327 & 861 & 583 & 676 & & 2285 & 1691 & 32 & 50 \\
\hline & 2351 & 642 & 922 & 990 & & & & & \\
\hline 2100 & 2045 & 9434617 & 4 & 18636 & & & & & \\
\hline & 2107 & 852816 & 20 & 8298 & & & & & \\
\hline & 2119 & 574935 & 25 & 6975 & & & & & \\
\hline & 2170 & 48717 & 156 & 3711 & & & & & \\
\hline & 2237 & 6721 & 66 & 243 & & & & & \\
\hline & 2253 & 4777 & 61 & 169 & & & & & \\
\hline & 2277 & 2357 & 206 & 344 & & & & & \\
\hline & 2308 & 1216 & 139 & 167 & & & & & \\
\hline & 2324 & 778 & 635 & 676 & & & & & \\
\hline & 2351 & 548 & 972 & 990 & & & & & \\
\hline & 2385 & 485 & 960 & 970 & & & & & \\
\hline
\end{tabular}


Table 2.2: Tabular data for Fig. 2.5 (Lifetime) Characteristic decay time $\tau$ from experiment (jet perturbation) as a function of Reynolds number $R e$ and $t_{0}=0$. Each row corresponds to a set of measurements. The fourth and fifth columns indicate the number of decaying events $r$ and total number of runs $n$.

\begin{tabular}{lllll}
\hline$L$ & $R e$ & $\tau$ & $r$ & $n$ \\
\hline $\mathbf{3 3 8 0}$ & 2015 & 1014588 & 67 & 20052 \\
& 2032 & 9262569 & 22 & 59773 \\
\hline
\end{tabular}

Table 2.3: Tabular data for Fig. 2.5 (DNS) Characteristic splitting time $\tau$ from direct numerical simulations as a function of Reynolds number $R e$. The second and third columns indicate the number of splitting events $r$ and total number of runs $n$. The runs were terminated after registering a splitting or after a truncation time of $t_{\max }$. The earliest splitting time for a set of runs is indicated as $t_{1}$. Upper and lower confidence intervals $\pm \Delta \tau$ are given in columns 7 and 8 , respectively are generated using $B=10^{5}$ bootstrap samples.

\begin{tabular}{lllllllll}
\hline & $R e$ & $\tau$ & $r$ & $n$ & $t_{1}$ & $t_{\max }$ & $+\Delta \tau$ & $-\Delta \tau$ \\
\hline DNS1 & 2275 & 1795 & 15 & 30 & 281 & 1500 & 1349 & 664 \\
& 2300 & 1042 & 22 & 30 & 177 & 1500 & 550 & 344 \\
& 2325 & 563 & 27 & 30 & 233 & 1500 & 247 & 184 \\
& 2350 & 445 & 48 & 50 & 170 & 1500 & 133 & 109 \\
& 2375 & 454 & 29 & 30 & 147 & 1500 & 153 & 116 \\
DNS2 & 2250 & 5696 & 12 & 50 & 410 & 2000 & 6465 & 2269 \\
& 2300 & 1923 & 29 & 60 & 194 & 1500 & 965 & 572 \\
& 2350 & 484 & 42 & 45 & 130 & 1500 & 165 & 130 \\
\hline
\end{tabular}





\section{High-precision Taylor-Couette experiment}

Citation and credit: Reprinted with permission from Review of Scientific Instruments, volume 84, article 065106, 6th June 2013, DOI: 10.1063/1.4807704

Copyright 2013, AIP Publishing LLC.

Title: 'High-precision Taylor-Couette experiment to study subcritical transitions and the role of boundary conditions and size effects'

Authors: K. Avila and B. Hof

Contribution: I designed and built the corresponding experiment (with the support of an external engineering office). I wrote the paper (the introduction was polished by B. Hof, minor comments about the rest), decided on the illustrations from the design and prepared them with annotations as figures. I performed all measurements, analyzed them and made all figures. Although the main focus of the paper is a technical description of the setup, the measurements included in the paper (stability diagram) shed a new light on the subcritical transition to turbulence in Taylor-Couette flows.

\subsection{Abstract}

A novel Taylor-Couette system has been constructed for investigations of transitional as well as high Reynolds number turbulent flows in very large aspect ratios. The flexibility of the setup enables studies of a variety of problems regarding hydrodynamic instabilities and turbulence in rotating flows. The inner and outer cylinder and the top and bottom endplate can be rotated independently with rotation rates of up to $30 \mathrm{~Hz}$, thereby covering five orders of magnitude in Reynolds numbers $\left(R e=10^{1}-10^{6}\right)$. The radius ratio can be easily changed, the highest realized one is $\eta=0.98$ corresponding to an aspect ratio of 260 gap width in the vertical and 300 in the azimuthal direction. For $\eta<0.98$ the aspect ratio can be dynamically changed during measurements and complete transparency in the radial direction over the full length of the cylinders is provided by the usage of a precision glass inner cylinder. The temperatures of both cylinders are controlled independently. Overall this apparatus combines an unmatched variety in geometry, rotation rates and temperatures, which is provided by a sophisticated high-precision bearing system. Possible applications are accurate studies of the onset of turbulence and spatio-temporal 
intermittent flow patterns in very large domains, transport processes of turbulence at high $R e$, the stability of Keplerian flows for different boundary conditions and studies of baroclinic instabilities.

\subsection{Introduction}

\subsubsection{Applications of the Taylor-Couette setup}

Taylor-Couette flow is the flow of fluid in the gap between two concentric rotating cylinders and was first devised more than a century ago by Mallock and Couette in order to determine the viscosity of fluids (Mallock 1888, 1896, Couette 1890). Since then, the flow in this geometry has become one of the most widely studied problems in fluid dynamics, particularly for investigations of hydrodynamic stability, the onset of chaos and turbulence (Cross \& Hohenberg 1993). Taylor demonstrated that the onset of the primary centrifugal instability could be accurately predicted by linear stability analysis (Taylor 1923). With increasing rotation rate of the inner cylinder the flow undergoes various transitions and exhibits a great variety of flow states while it gradually becomes turbulent (Coles 1965).

Similarly if both cylinders are rotated (either co or counter rotation) and the gap (and hence curvature) is large the flow is still dominated by the centrifugal instability followed by bifurcation scenarios leading to chaos. The majority of Taylor-Couette studies were concerned with such supercritical transition scenarios (Andereck et al. 1986, DominguezLerma et al. 1986), including their dependence on finite-size effects (Benjamin 1978, Heise et al. 2009). This transition type bears many similarities to Rayleigh-Benard convection, which is thermally driven fluid confined between a hot bottom and a cold top plate. Recently a large Taylor-Couette facility, the Twente-Turbulent-Taylor-Couette experiment has been built to compare transport properties and further explore analogies between these two flows in the turbulent regime (van Gils et al. 2011a).

A qualitatively very different transition scenario is found in Taylor-Couette (TC) if the gap is small and the outer cylinder rotates faster than the inner one. In this case the flow typically goes directly from laminar to turbulent and this transition already occurs in regimes where the laminar flow is linearly stable (hence resulting from finite amplitude perturbations). From the theoretical side much less is known about this transition type because standard stability analysis fails here. Experimental studies of this regime on the other hand are technically very challenging because perturbation levels have to be kept low (to avoid uncontrolled transition) while at the same time the gap needs to be small.

Overall only very few experiments addressed this regime. What has been found so far is that right after transition turbulence is spatially intermittent and typically only fills part of the flow domain. Flows can either consist of alternating bands of laminar and turbulent motion, known as spiral or barber-pole turbulence (Coles 1965), or of isolated transient spots surrounded by laminar flow (Borrero-Echeverry et al. 2010). Coles already noted that 'these are the same properties which distinguish the pipe flow' (Coles 1965). In pipes at low Reynolds numbers turbulence appears in the form of localized structures (Reynolds 1883) (called puffs in pipe flow) which are transient and decay after a finite time (Hof et al. 2006, 2008, Avila et al. 2010, Kuik et al. 2010). However, the ability of such puffs to spread and split thereby generating new puffs can statistically outweigh 
the decay of individual puffs, leading to a sustainment of turbulence (Avila et al. 2011, Barkley 2011). This non-equilibrium phase transition between transient and sustained turbulence takes place at Reynolds number $R e=2040 \pm 10$ (Avila et al. 2011) and is believed to be of second order (Barkley 2011, Allhoff \& Eckhardt 2012).

How these recent findings in pipe flow can be related to Taylor- and plane Couette flows is not yet understood. One of the main differences being that turbulence cannot only spread in the stream-wise but also in the span-wise direction, leading to spatial intermittency in two dimensions. The transition scenario is thus likely to be more complicated.

Earlier investigation of two dimensional intermittent flow patterns were carried out in plane Couette flow (Bottin \& Chaté 1998, Duguet et al. 2010). From measurements of the mean turbulent fraction as a function of $R e$ the authors inferred that (in contrast to pipe flow) here the transition is of first order. However, the recent studies of pipe flow (Avila et al. 2011) infer that equilibrium turbulent fractions can only be determined accurately if domain sizes are much larger than previously expected. Therefore it is also not clear if the system size was sufficient in these earlier Couette experiments to unambiguously determine the nature of this transition. Unfortunately large plane Couette experiments are technically difficult to control (Bottin \& Chate 1998) with deviations of the order of $7 \%$ (Prigent et al. 2003), and numerical simulations that would match the time scales used in the experiments are currently too expensive to allow statistical studies (Duguet et al. 2010). Another possibility to still study this transition is to use a TC experiment, which is easier to control (including the boundary condition) and offers all the advantages of a closed system. In the TC system the azimuthal length (at midgap) $L_{\text {azim }}$ depends on the radius ratio $\eta$ of the two cylinders $\left(L_{\text {azim }}=\pi(\eta+1) /(1-\eta)\right)$ : the larger the radius ratio the larger the azimuthal length. The limit of $\eta=1$ corresponds to plane Couette flow and hence for flows in narrow gap experiments with a faster outer cylinder speed the transition becomes subcritical just like in plane Couette flow (Faisst \& Eckhardt 2000, Prigent et al. 2002). However as $\eta$ approaches the value of one not only the flow states resemble each other, but also some of the technical problems. As the gap-width in highradius ratio TC systems is very small compared to the radii of the cylinders, any small geometric imperfection of either cylinder may produce a substantial loss of accuracy in the gap width and hence influence the dynamics (Schultz-Grunow 1959). For example, the super-critical onset of Taylor vortices in an $\eta=0.99$ experiment could only be determined with an accuracy of $20 \%$ in Re (Carey et al. 2007). In the subcritical regime the precision of the apparatus is even more important: imperfections may induce finite amplitude perturbations that already trigger turbulence in an uncontrolled manner and this would make measurements of characteristic quantities in the asymptotic limit (e.g. equilibrium turbulent fraction) of the idealized system impossible. Such problems occurred in previous plane Couette experiments and high-radius ratio TC setups $(\eta=0.983)$ (Prigent et al. 2003) (see Section 3.5.2). The purpose of this paper is to show that it is nevertheless possible to built a TC apparatus overcoming these challenges and allowing to study the nature of the subcritical phase transition between decaying and spreading turbulence in a large system of 311 (azimuthal) x 263 (axial) gap width $(\eta=0.98$ ).

In recent years TC flows have been used to study the stability of quasi-Keplerian velocity profiles. Here the azimuthal velocity component decreases in the radial direction (with $r^{-1.5}$ for Keplerian), whereas the angular momentum increases. Flows of this type occur in astrophysical accretion discs and following a criterion by Lord Rayleigh it is well 
known that such velocity profiles are linearly stable (Rayleigh 1917). However, observations in astrophysics infer that such flows must be turbulent because the observed accretion rates are orders of magnitude shorter than one would expect from laminar (molecular) transport. So there must be a physical mechanism leading to instability and resulting in higher (turbulent) angular momentum transfers. The TC setup offers the possibility to generate a quasi-Keplerian velocity profile when both cylinders are co-rotating at adequate speeds but the challenge is here to minimize the effects of the axial endplates on the flow. A recent numerical study by M. Avila has shown that the endplates in current experiments severely distort the flow profile and trigger instabilities already at Reynolds numbers at about 1000 (Avila 2012). One of the current experiments (Princeton) has each endplate split into two rings that can be rotated independently and therefore adapted to the desired velocity profile (Ji et al. 2006, Schartman et al. 2009). However, the aspect ratio $\Gamma=2.1$ is so small, that the end wall effects are necessarily very large at higher $R e$. The aspect ratio $\Gamma$ is defined as $L / d$ with the gap length $L$ and the gap width $d$. The other experiment (Maryland) has an aspect ratio of $\Gamma=11.5$, but the endplates are attached to the outer cylinder (Paoletti \& Lathrop 2011) as this is also the case for many other experiments (Atlanta (Borrero-Echeverry et al. 2010), Delft (Ravelet et al. 2010), Twente (van Gils et al. 2011b)). To minimize end wall effects and to be able to generate Keplerian type flows at higher $R e$ it is desirable to combine movable end walls and large aspect ratios. Our TC setup offers the possibility of independently rotating top and bottom endplates, to change the aspect ratio continuously during measurements and to investigate the flow for different radius ratio of the cylinders for $R e$ ranging from $10^{1}$ up to $10^{6}$. In addition, in contrast to most of the experiments used in this regime we have full optical access to the flow.

The paper is structured as follows. In Section 3.2.2 we list the main features of the experiment and summarize the achieved accuracy, in Section 3.3 it is explained in detail how this was achieved, with the focus on the bearings. In Section 3.4 we give an example of the image processing from flow visualization, which will be used to investigate the subcritical phase transition. First measurements of the stability are compared with theory and previous experimental investigations in Section 3.5. An outlook and discussion is given in Section 3.6.

\subsubsection{High precision Taylor-Couette apparatus}

The Göttingen TC experiment is designed to cover a huge parameter space in Reynolds number and radius ratio, combined with the flexibility to dynamically change the aspect ratio and the axial boundary conditions by rotating the endplates independently. In order to study subcritical transitions also in a high radius ratio the experiment has to be as precise as possible to avoid triggering finite amplitude perturbations. In the following we list the main features:

- The inner and outer cylinder and the top and bottom endplate rotate independently up to $30 \mathrm{~Hz}$

- The possible radius ratio is between 0.03 and 0.98 , the already existing inner cylinders correspond to $\eta=[0.71,0.88,0.98]$ 


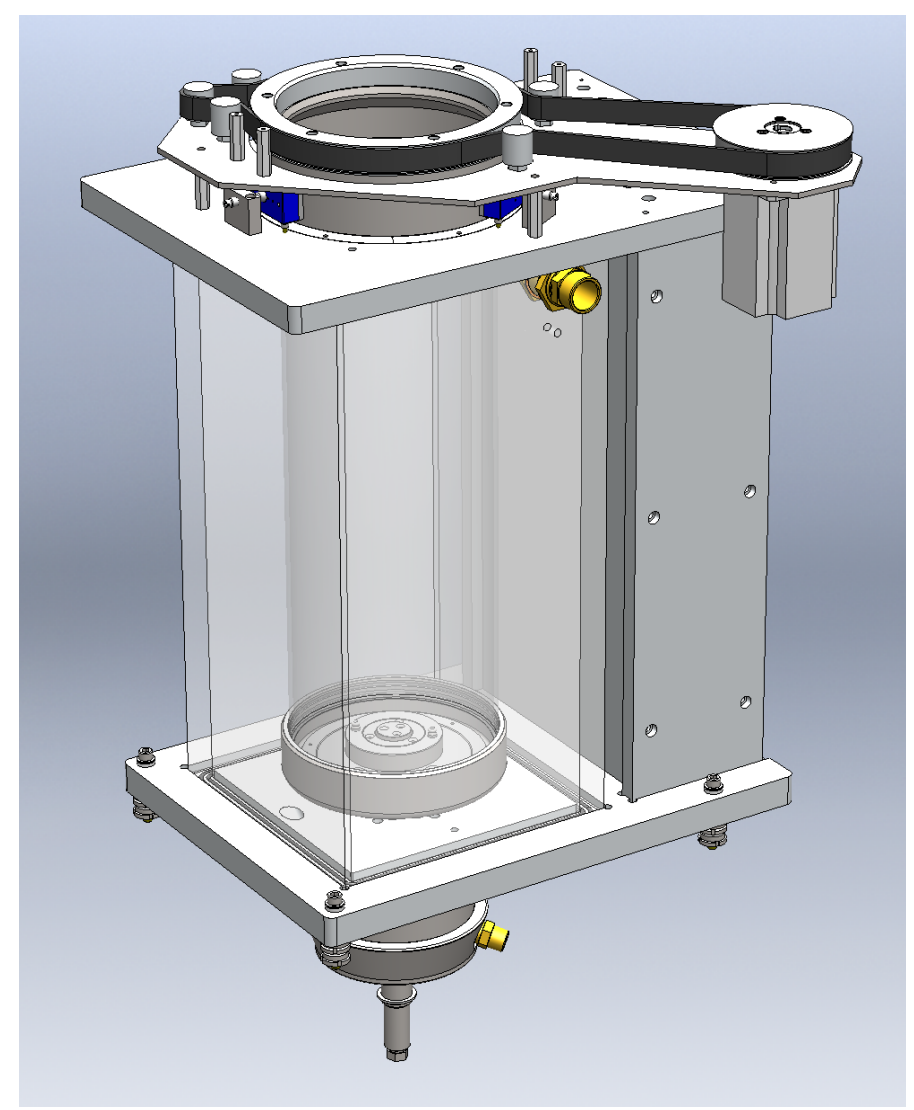

Figure 3.1: The outer cylinder is placed in a square acrylic box filled with silicone oil to provide index-matching and temperature stability. On top and bottom the acrylic box is mounted on massive aluminum plates that are in addition connected by a pillar. The pillar and the aluminum plates build the frame on which the bottom bearing unit and the drive (and bearing) of the outer cylinder is mounted. Altogether this assembly group contains both cylinders and the bottom endplate.

- The aspect ratio can be continuously changed during measurements from its maximum value to zero (except for $\eta=0.98$ ).

- The system is completely transparent in the radial direction over the full length of the cylinders to optimize the access for optical measurements. The outer and inner cylinder are made of glass (except for $\eta=0.98$ ). To match the refractive index the inner cylinder is filled with silicone oil and the outer cylinder is placed in a square box of acrylic filled also with silicone oil.

The temperature stability $( \pm 0.01 \mathrm{~K})$ and rotation rates (deviation $\leq 1 \%)$ are precisely controlled and all rotating assembly groups have been dynamically balanced to a precision of $\leq 1 \mathrm{~mm} / \mathrm{s}$ corresponding to a balance quality grade $\mathrm{G}=1$ (ISO $1940^{1} \mathrm{]}$ ), which is

\footnotetext{
${ }^{1}$ Mechanical vibration - Balance quality requirements for rotors in a constant (rigid) state - Part 1 : Specification and verification of balance tolerances, International Organization for Standardization, Geneva, Switzerland, 2003
} 


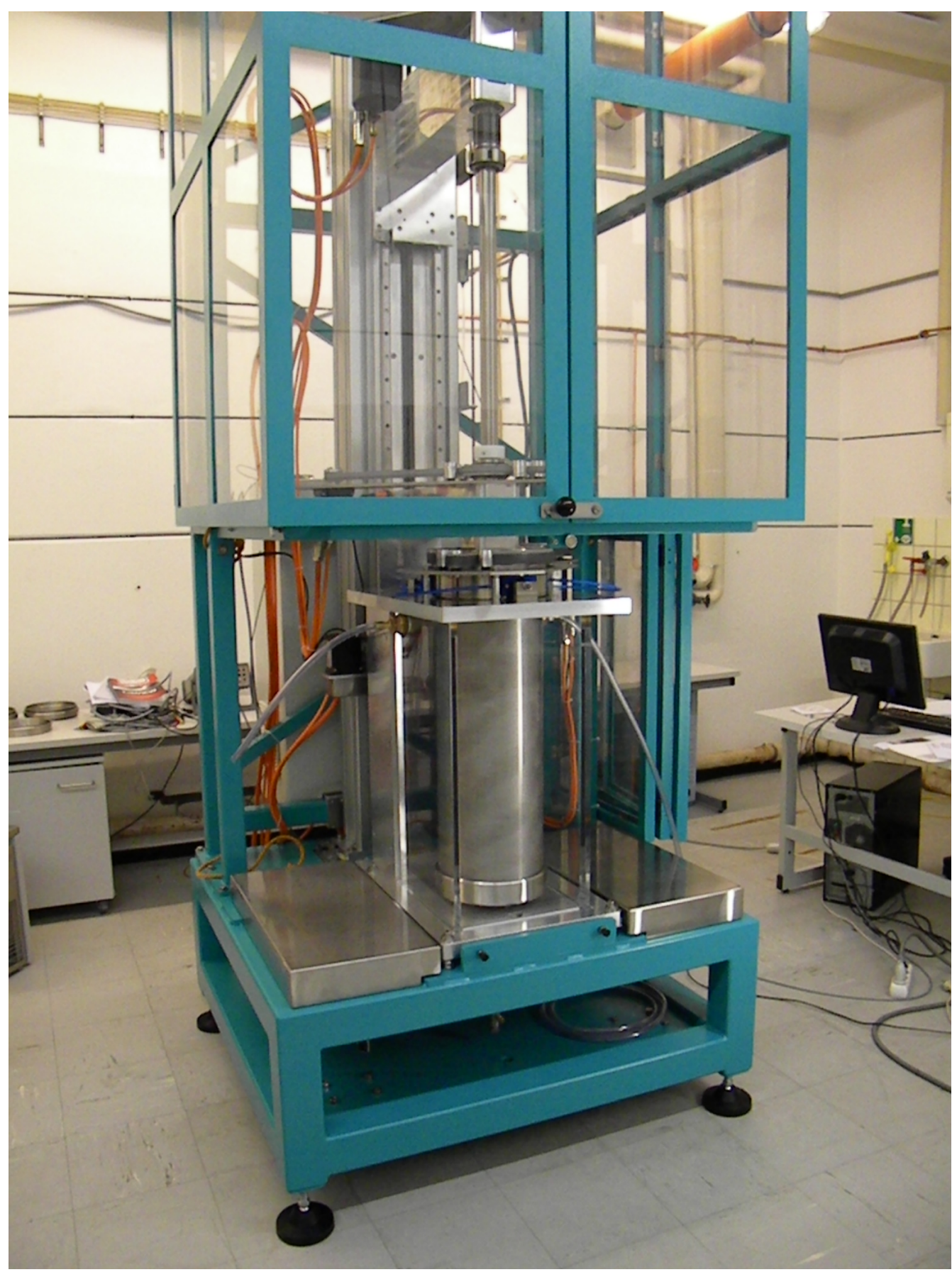

Figure 3.2: The Taylor-Couette device consists of three main assembly groups. The first group consists of the cylinders, the bottom endplate and the acrylic box (see Fig. 3.1). The second group allows an axial movement of the top endplate during measurements over the full length of the cylinders. It consists of a heavy-lift profile along which two massive sledges can be moved, that bear the top endplate and the top of the inner cylinder (see Fig. 3.5). The third assembly group is a stiff frame (here shown in green) on which the first and second assembly groups are mounted and aligned with respect to each other. 


\begin{tabular}{crc} 
Radius ratio & $\begin{array}{r}\text { Gap width } d \\
(\mathrm{~mm})\end{array}$ & $\begin{array}{r}\text { Deviation of } d \\
(\%)\end{array}$ \\
\hline$\eta=0.71$ & $32.53 \pm 0.210$ & 0.65 \\
$\eta=0.88$ & $13.53 \pm 0.140$ & 1.04 \\
$\eta=0.98$ & $2.28 \pm 0.075$ & 3.29
\end{tabular}

Table 3.1: Geometric dimensions of the gap for different radius ratio and the achieved precision. The deviations are measured while being mounted in the experiment. The axial runout of the endplates is within $\pm 0.030 \mathrm{~mm}$, corresponding to a deviation of the maximal gap length $L$ of less than $0.01 \%$. Further details are summarized in Table 3.2.

typically achieved by machine tools. In publications it is common to describe the geometric precision of a TC-experiment by referring to the deviation of the cylindricity of the cylinders given by the manufacturer (Prigent \& Dauchot 2000b, Ravelet et al. 2010, van Gils et al. 2011b). Few experiments also take account of the deviations stemming from the bearing (Schartman et al. 2009). We find that the values of precision given even by world-leading manufacturers (e.g. Schott Duran) are locally often exceeded. In order to estimate all geometric deviations possibly acting on the flow we have measured and optimized all mechanical components while being mounted in the experiment. Table 3.1 summarizes the physically relevant measurements.

A comparison of the non-dimensionalized system size of our setup with other currently in use TC experiments is given in Table 3.3, including the axial boundary conditions.

\subsection{System Description}

\subsubsection{General setup}

The Taylor-Couette (TC) apparatus consists of two vertical, concentrically aligned cylinders with different diameters, which can be rotated independently. The gap between the two cylinders is filled with a working fluid (typically silicone oil or water) to investigate the flow states depending on the speed of the cylinders using optical measurement techniques. Therefore the outer cylinder is made of glass producing full optical access over the whole gap. The outer cylinder is mounted between aluminum plates at top and bottom and placed inside a square acrylic box. The entire box is filled with a temperature controlled fluid (silicone oil or water) to provide temperature stability and a matching of the refractive index. To avoid stresses in the acrylic box, the two aluminum plates are in addition connected by an aluminum pillar $(350 \mathrm{~mm} \times 180 \mathrm{~mm})$ as it is illustrated in Fig. 3.1.

The working fluid inside the gap is confined in the axial direction by annular endplates, that can be rotated independently. The top endplate can in addition be moved in the axial direction over the full length of the cylinders of $600 \mathrm{~mm}$ (except for $\eta=0.98$ ), allowing to change the aspect ratio of the gap continuously. The axial movement of the endplate is driven by a motor that moves a sledge along a high-precision profile that is mounted on a heavy-lift profile. The top bearing of the inner cylinder is also mounted on this profile so 
Radial runout (mm)

\begin{tabular}{crrrr}
\hline Position of measurement & Outer cylinder & \multicolumn{3}{c}{ Inner cylinder } \\
$L$ & & $\eta=0.98$ & $\eta=0.88$ & $\eta=0.71$ \\
\hline 0 (bottom) & 0.025 & 0.025 & 0.050 & 0.110 \\
$1 / 4$ & 0.050 & 0.021 & 0.045 & 0.115 \\
$1 / 2$ & 0.045 & 0.021 & 0.070 & 0.125 \\
$3 / 4$ & 0.050 & 0.021 & 0.075 & 0.130 \\
1 (top) & 0.050 & 0.023 & 0.090 & 0.160 \\
Overall accuracy of $r_{i, o}{ }^{1}$ & $112.53 \pm 0.050$ & $110.25 \pm 0.025$ & $99.00 \pm 0.090$ & $80.00 \pm 0.160$ \\
Bottom endplate & Axial runout (mm) \\
Top endplate & \multicolumn{4}{c}{0.030} \\
\end{tabular}

Table 3.2: Geometric dimension and precision of the cylinders and the endplates while they are mounted in the Taylor-Couette device. The runouts have been measured by a dial extensometer with a resolution of $0.002 \mathrm{~mm}$. Note, that for each radius ratio $\eta$ a corresponding pair of endplates is necessary.

1 absolute value measured by the manufacturer

that the top endplate and the top bearing of the inner cylinder are always concentric to each other (see Fig. 3.2 and Section 3.3.3.2 on page 69 for details). The challenge now is to align the top endplate (and therefore the top bearing of the inner cylinder) concentrically to the bottom bearing unit (consisting of the bottom endplate and the bottom bearing of the inner and outer cylinder, see Section 3.3.3.1 for details). Since the top endplate is guided along the heavy-lift profile in the axial direction, this profile and the cylinders have to be exactly parallel. Only in this case both endplates and both cylinders are concentric to each other at any axial position. For this purpose the heavy-lift profile can be moved in the horizontal plane and the box in which the bottom bearing unit is mounted can be aligned in three directions. Both components are mounted on a frame (shown in green in Fig. 3.2) with high stiffness that is aligned with respect to gravity.

\subsubsection{Geometry and materials of the cylinders and the endplates}

The outer cylinder is made of Borosilicate precision glass with a length of $650 \mathrm{~mm}$, a thickness of $5 \mathrm{~mm}$ and was manufactured to a radius at the inner surface of $r_{o}=112.53 \mathrm{~mm} \pm$ $0.02 \mathrm{~mm}$. We used glass in order to provide full optical access combined with a high precision, thereby also avoiding bending effects as they may appear with an acrylic cylinder at high rotation rates when no additional radial support is provided. The cylinder was manufactured by Duran Schott, which is to our knowledge the only company producing high-precision glass cylinders at this size. For larger diameters the production procedure changes and the precision is substantially decreased. To provide access for the endplates to enter the gap the outer cylinder is beared from the outside. Therefore the inner and outer cylinder surfaces need to be as concentric as possible to allow for a uniform rotation of the inner surface. The radial offset of the cylindricity centers between the inner and the outer cylinder surface was measured to be $0.103 \mathrm{~mm}$ which is double the value given by the company. It was improved by re-engineering the bearing seat at the outside of the outer cylinder. The overall achieved precision when the outer cylinder is mounted in the experiment is $r_{o}=112.53 \mathrm{~mm} \pm 0.050 \mathrm{~mm}$, corresponding to a deviation of $0.044 \%$.

The radius ratio $\eta$ of the cylinders can be chosen between 0.03 and 0.98 , which cor- 


\begin{tabular}{|c|c|c|c|c|c|c|c|c|}
\hline & Princeton & Cottbus & Twente & Maryland & Delft & Madison & Gif sur Yvette & Göttingen \\
\hline \multirow{3}{*}{$\Gamma=L / d$} & \multirow{3}{*}{2.1} & \multirow{3}{*}{20} & 11.6 & \multirow{3}{*}{11.7} & \multirow{3}{*}{22} & \multirow{3}{*}{44.8} & \multirow{3}{*}{430} & $0-18.4$ \\
\hline & & & 15.5 & & & & & $0-44.3$ \\
\hline & & & 23.3 & & & & & 263 \\
\hline \multirow{3}{*}{$\eta=r_{i} / r_{o}$} & \multirow{3}{*}{0.35} & \multirow{3}{*}{0.5} & 0.71 & \multirow{3}{*}{0.73} & \multirow{3}{*}{0.92} & \multirow{3}{*}{0.99} & \multirow{3}{*}{0.983} & 0.71 \\
\hline & & & 0.77 & & & & & 0.88 \\
\hline & & & 0.83 & & & & & 0.98 \\
\hline \multirow{3}{*}{$L_{a z i m}^{1}$} & \multirow{3}{*}{6.5} & \multirow{3}{*}{9.4} & 18.5 & \multirow{3}{*}{20.1} & \multirow{3}{*}{75.4} & \multirow{3}{*}{625.2} & \multirow{3}{*}{366.5} & 18.5 \\
\hline & & & 24.2 & & & & & 49.2 \\
\hline & & & 33.8 & & & & & 311.0 \\
\hline $\begin{array}{l}\text { Endplate } \\
\text { condition }\end{array}$ & $\begin{array}{l}\text { independent, } \\
\text { two rings }\end{array}$ & stationary & & ed to outer & linder & $\begin{array}{c}\text { attached to } \\
\text { outer cylinder }\end{array}$ & not described & independent \\
\hline
\end{tabular}

Table 3.3: Comparison of the non-dimensionalized geometries of various Taylor-Couette devices with both cylinders rotating (except for Madison), operating either at high Reynolds numbers (Princeton, Cottbus, Twente, Maryland) or in a high radius ratio (Delft, Madison, Gif sur Yvette) and their axial boundary condition.

References: Princeton (Schartman et al. 2009), Cottbus (Merbold et al. 2013), Twente (van Gils et al. 2011b), Maryland (Paoletti \& Lathrop 2011), Delft (Ravelet et al. 2010), Madison (Carey et al. 2007), Gif sur Yvette (Prigent \& Dauchot 2000b), Göttingen (Avila \& Hof 2013).

${ }^{1} L_{\text {azim }}=\pi(\eta+1) /(1-\eta)$

2 and free surface at the top 
responds to a radius of the inner cylinder $r_{i}$ between $3.38 \mathrm{~mm}$ and $110.25 \mathrm{~mm}$, currently built ratios are $\eta=[0.71,0.88,0.98]$. The inner cylinders are beared from the inside using top and bottom caps that have an identical fit for the supports for all inner cylinders. The same system is adapted to the endplates, so that the inner cylinder with its corresponding pair of endplates can be exchanged, without changing any alignment or further dismantling of the experiment. For $\eta=0.98$ the inner cylinder is made of stainless steel with a polished surface and an overall precision when mounted in the experiment of $r_{i}=$ $110.25 \mathrm{~mm} \pm 0.025 \mathrm{~mm}$ corresponding to a deviation of $0.023 \%$. The endplates have a width of $2.00 \mathrm{~mm}$, leaving a gap of minimum $50 \mu \mathrm{m}$ to each cylinder (including the radial runouts of cylinder and endplate). The axial position of the top endplate is fixed during measurements (for $\eta=0.98$ ), resulting in an aspect ratio $\Gamma=263$. For $\eta=0.88$ and 0.71 the inner cylinder are made of Borosilicate precision glass with a length of $650 \mathrm{~mm}$ to optimize the illumination and access for Stereo or Tomo-PIV measurements. The inside of the inner cylinder is filled with the working fluid (but without tracers) to avoid reflections and provide index matching in the radial direction. However, the advantages of transparency come with losses in precision as it was already described for the outer cylinder. Here the complete outside surface of the inner cylinders was re-engineered using a lathe (done by Schott Duran). The achieved accuracy is still worse than for the steel inner cylinder (see Table 3.2), but since the gap is much wider the relative deviation of the gap width (and therefore the Reynolds number) is with about $1 \%$ still smaller (see Table 3.1).

The top endplate can be continuously moved or kept at any axial position between the maximum gap length $L_{\max }=600 \mathrm{~mm}$ and $L_{\min }=0 \mathrm{~mm}$. Especially in small aspect ratio systems the axial runout of the endplates can considerably disturb the flow, even when the endplates are stationary (Pacheco et al. 2011). Besides the accuracy of the cylinders Table 3.2 includes therefore also the axial runout of the endplates. The gap between each cylinder and the endplates is on average about $0.15 \mathrm{~mm}$ to provide a friction free rotation or/and axial movement. The endplates are made of stainless steal.

\subsubsection{Bearing, sealing and balancing}

A sophisticated bearing system has been designed which allows for independent rotation of both cylinders and both endplates. At the same time the aspect ratio should be variable and both cylinders should be transparent for optical access. In addition the overall perturbation level has to be minimized to avoid transition to turbulence in the subcritical regime even in a high-radius ratio $(\eta=0.98)$. Examples of such perturbations are runouts of the cylinders and the endplates, variations of the rotation speeds or local heat sources. All these are strongly influenced by the arrangement of the ball bearings, the seals and their cooling. The geometric runouts of the cylinders and the endplates in Table 3.2 are mainly due to the limited accuracy of the components themselves while the deviations stemming from the bearings are much smaller (about $0.005 \mathrm{~mm}$ at the shafts). In other experiments clearances in the bearings cause the main deviations, especially when the setup is very complex (Schartman et al. 2009). The high accuracy in the present setup could be reached with the following design.

Each rotating assembly group is guided by two high-precision angular contact ball bearings (quality P3) arranged in O- or X-position along a vertical shaft and is dynami- 
cally balanced to a precision of $\leq 1 \mathrm{~mm} / \mathrm{s}$ corresponding to a balance quality grade $\mathrm{G}=1$ (ISO $1940^{2}$ ). Each component of an assembly group is measured for axial and radial runouts while being mounted in the experiment and is assembled to its neighbor in a way that runouts are minimized (compensated). Labeling assures an identical arrangement even after dismantling. Accuracy changes after complete dismantling of the experiment are within $\pm 0.005 \mathrm{~mm}$ (measured at the cylinders and endplates). All rotating components (shafts, flange) are made of stainless steel and have tight fittings with a radial and axial runout below $\pm 0.005 \mathrm{~mm}$ and a high surface quality (roughness parameter $R a=0.8$ ). Each pair of ball bearings is build in with a permanent bearing-preload using belleville spring packs to provide a backlash-free run and low friction. The driving force of the motor is transmitted without generating any radial force on the shafts (see Section 3.3.5 on page 72 for more details).

While all these aspects apply to the bearing at the top and the bottom of the experiment, there are also various differences. At the bottom three independently rotating assembly groups had to be housed within each other with a high precision, thereby separating two different fluid volumes by seals that have to be cooled. The design on the upper part was complicated by the requirement that the (rotating) top endplate must be mobile in the axial direction over the full length of the cylinders with a high accuracy (see Table 3.2). In addition changing the inner cylinder and its corresponding pair of endplates should be as simple as possible with a minimum of re-alignment. To minimize friction, no seals were used at the top bearing. In the following sections we describe the top and bottom bearing in more detail.

\subsubsection{Bottom bearing unit}

The bottom bearing unit is a concentric shaft system for the outer cylinder, for the bottom endplate and for the inner cylinder as can be seen in Fig. 3.3. The fittings are very tight so that single components have to be heated by a hot-air blower to (dis-)assemble them. The bottom endplate itself can be exchanged more easily, because it is beared in the radial direction by an O-ring (see Fig. 3.3). For an exchange it has just to be lifted and the new one pressed in until it meets the axial fitting. However, this system does not apply to the narrow gap endplate $(\eta=0.98)$ due to the limited space. Here the support with the O-ring has to be removed and the endplate is screwed directly to the support beneath (indicated by the arrow). Except for the small modification for the narrow gap endplate all endplates and inner cylinders have identical fittings and can thus be easily exchanged.

The total number of seals was reduced to two in order to avoid friction and thus heating: Seal 1 (see Fig. 3.3) to prevent mixing between the working fluid in the gap and the cooling liquid in the square box around the outer cylinder and another Seal 2 to prevent loss of cooling liquid from this box. We use low-friction seals made of Teflon that are surrounded by a cooling channel system (see Section 3.3.6 on page 75). The bottom of each support has a tilted surface to prevent air bubbles (that might be trapped in the working fluid after filling) entering the measurement gap in an uncontrolled manner. With this tilt air bubbles are immediately evacuated (i.e. before the measurement starts)

\footnotetext{
${ }^{2}$ Mechanical vibration - Balance quality requirements for rotors in a constant (rigid) state - Part 1: Specification and verification of balance tolerances, International Organization for Standardization, Geneva, Switzerland, 2003
} 


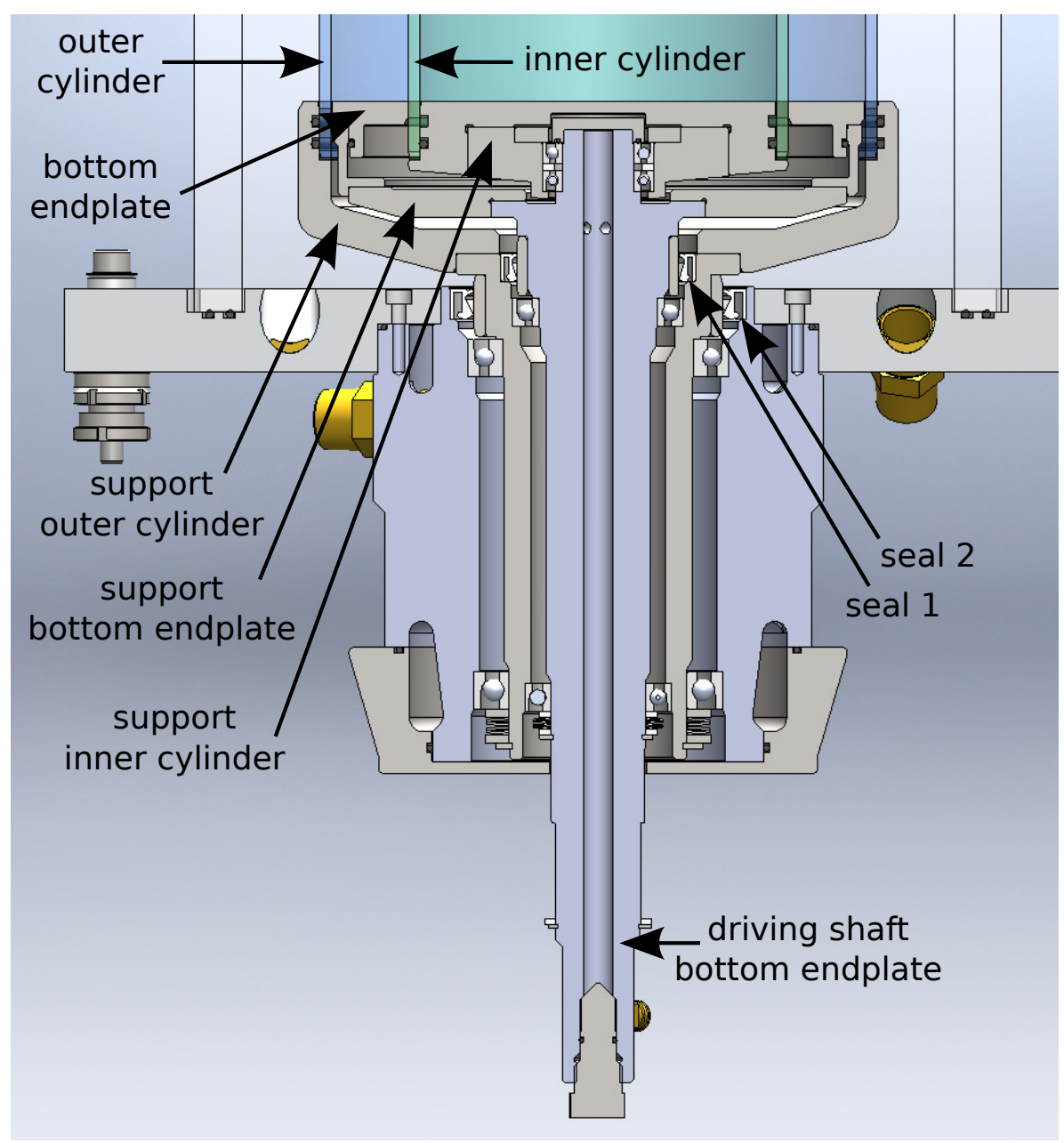

Figure 3.3: The bottom bearing unit houses the bottom of both cylinders and the bottom endplate. It is mounted on the aluminum plate beneath the acrylic box (see Fig. 3.1). Each rotating shaft is kept in position by two ball bearings that are separated by a maximal axial distance to optimize the alignment. Clearances of the bearings are minimized by tight radial fittings and an axial preload provided by belleville spring packs. The number of seals is minimized to two to avoid unnecessary friction. The inner cylinder (and its support) are not sealed to allow torque measurements. 
(a)

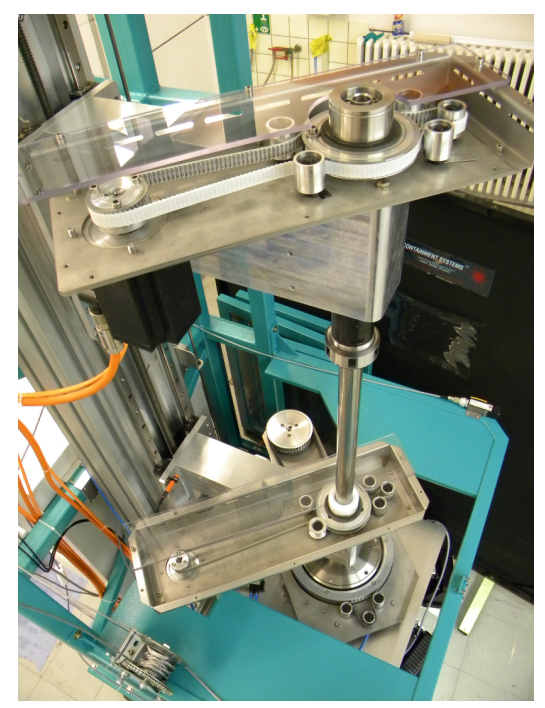

(b)

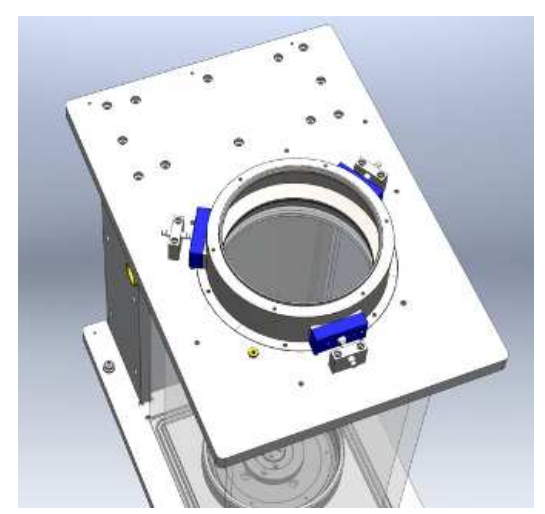

(c)

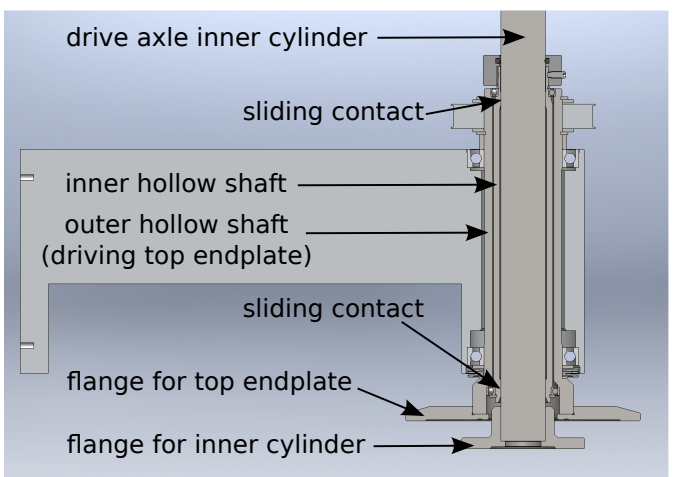

Figure 3.4: (a) View from the top at the TC-device to demonstrate the top bearings for the rotation. The two upper timing belts are clearly visible, that are each mounted on a sledge moving along the vertical heavy-lift profile. Below them the timing belt of the outer cylinder can be seen. It is mounted on a small plate on top of the aluminum plate which is on top of the acrylic box. Below the small plate is the top bearing of the outer cylinder: (b) The stainless steel ring bearing the outer cylinder at the top is concentrically mounted to the outer glass cylinder by two O-rings. The outer surface of this ring is polished and kept in position by an air bearing consisting of three concave blocks made of a porous media (shown in blue). By pumping pressurized air through this blocks the outer cylinder is beared frictionless and able to reach high rotation rates. The outer cylinder is concentrically aligned with respect to the inner cylinder by positioning the three blocks with respect to the aluminum plate. (c) The shaft of the inner cylinder and of the top endplate are beared in the lower sledge to provide concentricity. The design allows in addition to the two independently rotating shafts an axial movement of the top endplate during measurements (see Section 3.3.4 on page 70 for more details).

instead of hanging for a while and entering the measurement gap at unpredictable times. The working fluid in the gap can be emptied via the hollow driving shaft of the bottom endplate.

\subsubsection{Top bearing units}

The top bearing consists of three independent assembly groups. The first is housing the top of the outer cylinder, the second allows the axial movement and rotation of the top 
endplate and is in addition bearing the driving shaft of the inner cylinder that is passing through to provide concentricity. In the third assembly group the shaft of the inner cylinder is finally driven. In the following this shaft will be referred to as 'drive axle' or 'drive axle of the inner cylinder' to avoid confusion with other shafts. The second and third assembly group can be moved axially by being mounted on the heavy-lift profile that is aligned with respect to the cylinders (see Fig. 3.4(a) and Section 3.3.1).

The first assembly group to bear the outer cylinder shown in Fig. 3.4(b) is mounted on an aluminum plate which sits on top of the acrylic box and on an additional pillar connecting the top aluminum plate with the bottom aluminum plate (see Fig. 3.1). The outer cylinder is guided from the outside to have free access to the gap. Due to the potentially high rotation rates of the outer cylinder of $30 \mathrm{~Hz}$ (corresponding to an angular velocity at the bearing seat of $90 \mathrm{~km} / \mathrm{h}$ ) the bearing is via frictionless radial air bearings (New way by IBS Precision Engineering, concave L profile). The air bearing consists of three independent parts that can be moved in the radial direction to align the top of the outer cylinder concentrically to the bottom part (and therefore also to the inner cylinder and both endplates).

The second assembly group guiding the top endplate and the drive axle of the inner cylinder consists of a massive bearing support made of aluminum, two hollow shafts, the drive axle and two high accuracy flanges made of stainless steal as shown in Fig. 3.4(c). The top endplate with the chosen geometry is directly attached to the outer flange for $\eta=0.98$ or to a cylinder with a length of $600 \mathrm{~mm}$ for the other radius ratios (see Fig. 3.5). This outer flange is permanently connected to the outer hollow shaft (mounted by heatshrinking and afterwards dressed to size). It is guided by two high-precision angular contact ball bearings in $\mathrm{O}$-arrangement. The outer hollow shaft is driven by a toothed pulley to rotate the top endplate. The inner hollow shaft is beared in the outer hollow shaft by high-precision deep groove ball bearings to provide concentricity. The inner hollow shaft is in sliding contact with the drive axle from the inner cylinder. These two components slide against each other when the top endplate is axially moved (by moving the sledge as a whole). Here the sliding friction is artificially increased, so that during (pure) rotation the drive axle of the inner cylinder is rotating together with the inner hollow shaft (which is guided by deep groove ball bearings).

The drive axle of the inner cylinder is massive and made of stainless steel with a length of $1000 \mathrm{~mm}$, a diameter of $40 \mathrm{~mm}$ and a polished surface. At the bottom end it has a flange (mounted by heat-shrinking and afterwards dressed to size) that fits in the top caps of the various inner cylinders. At the top it is connected to a collet chuck shaft joining that is beared in the third assembly group and allows the driving of the inner cylinder. The third assembly group is similar to the second one but with just a single hollow shaft.

\subsubsection{Aspect ratio control}

For radius ratios other than 0.98 the top endplate can be continuously moved or kept at any axial position between the maximum gap length $L_{\max }=600 \mathrm{~mm}$ and $L_{\min }=0 \mathrm{~mm}$. Therefore the massive bearing support of the top endplate is screwed to a ball-beared sledge that moves along a high-precision profile that is mounted on the heavy-lift profile (see Fig. 3.5). The sledge is driven by a trapezoidal screw (slope: $5 \mathrm{~mm} /$ rotation) with self-locking that is connected to the timing belt of a brushless servo motor. The axial speed 


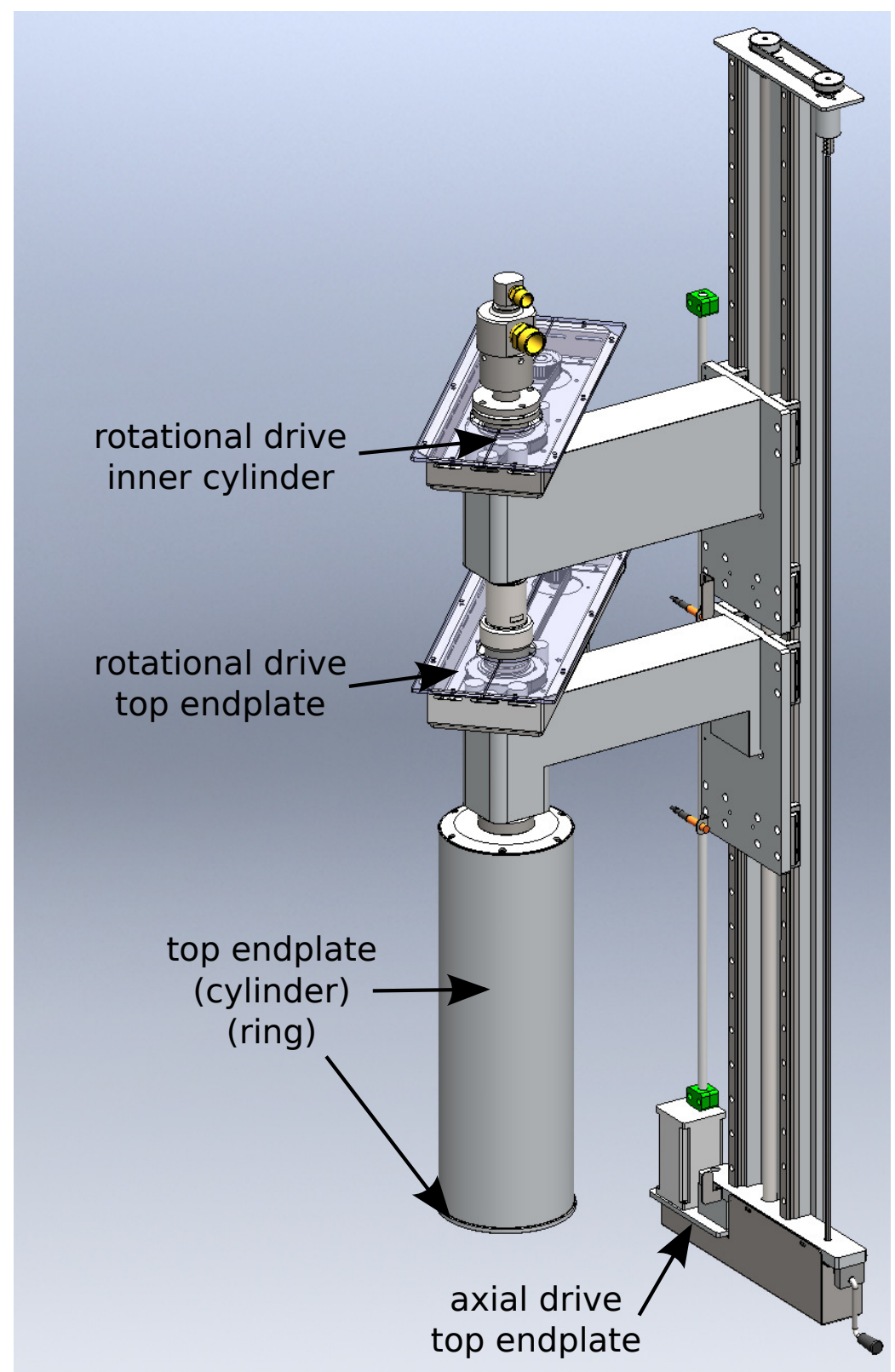

Figure 3.5: The bearing and drives of the top endplate and of the top part of the inner cylinder are mounted on sledges that can be moved vertically along a high-precision profile. The axial position of the lower sledge determines the axial position of the top endplate and therefore also the aspect ratio $\Gamma$ of the gap. The top endplate consists of a long cylinder and a short ring beneath fitting exactly in the gap between the cylinders. Its position can be dynamically changed during measurements by a servomotor. The top sledge with the rotational drive of the inner cylinder can be moved axially via a crank handle (see bottom right) for maintenance work or an exchange of the inner cylinder. 


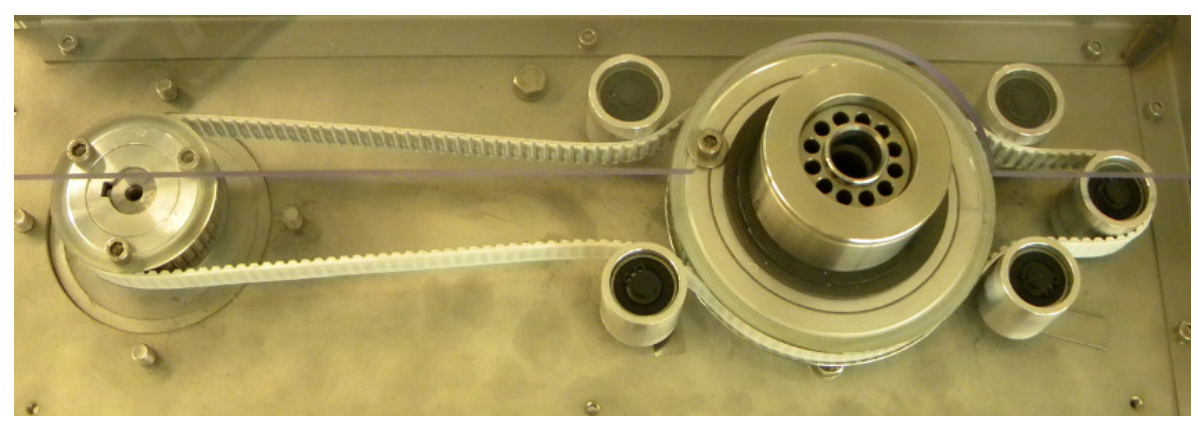

Figure 3.6: A special arrangement of the guidance of the timing belt by five rollers allows a transmission of the power from the servomotor (left side in the picture) to a rotating shaft (here of the inner cylinder) without generating a radial force on the shaft. This arrangement minimizes forces on ball bearings and thus runouts of the shaft in the longterm.

is in between $0.15 \mathrm{~mm} / \mathrm{s}$ and $35 \mathrm{~mm} / \mathrm{s}$, the position is measured by a digital glass scale with a resolution of $0.001 \mathrm{~mm}$ that is directly mounted on the sledge of the top endplate and the heavy-lift profile. Magnetic proximity sensors avoid collisions by stopping the servo motor. The rotation of the endplates and both cylinders is independent from the axial movement or position of the top endplate, so the aspect ratio can be continuously changed during the measurement. The realized aspect ratios $\Gamma$ are summarized in Table 3.3 .

\subsubsection{Rotation rate control}

Both cylinders and the endplates have a maximum rotation rate of $30 \mathrm{~Hz}$ and are driven by servomotors. The power is transmitted by a timing belt with toothed pulleys with a gear ratio of 3 for the endplates and 2.22 for the cylinders. In order to avoid radial forces on the driving shafts (of the cylinders and endplates) and therefore a loss of precision in the long-term use, each timing belt is guided by five fixed rollers as shown in Fig. 3.6. The tension and exact position of the timing belt is adjusted by aligning the asymmetric base of the fixed rollers.

The servomotors are brushless AC motors with permanent magnets and 16 Bit resolvers. To allow large accelerations the Novotron motors driving the cylinders are more powerful $(2.9 \mathrm{~kW}, 5.2 \mathrm{Nm}$, type NHDM 105E10-81SC47F00K10) than the ones for the endplates $(1.5 \mathrm{~kW}, 1.6 \mathrm{Nm}$, type NHDM 82C8-28SC0F00K10). The rotation rates are controlled in a feedback loop by the corresponding converters from Novotron (ND425610VD-011-000, ND41-3207VD-011-000), based on a proportional-integral-derivative (PID) control algorithm. The converters are connected to a PC by a serial port to operate the experiment and read the actual speeds of the motors.

In Fig. 3.7(a) the raw data signal from the fluctuations of the speed of the motor rotating at $5 \mathrm{~Hz}$ is shown by the black line. The signal includes electronic noise and a systematic geometric deviation of the resolver which is explained in the following: The speed of the rotor shaft is calculated from the change of its angular position with time. The position is detected by the resolver from the magnetic coupling of two transformers, which 


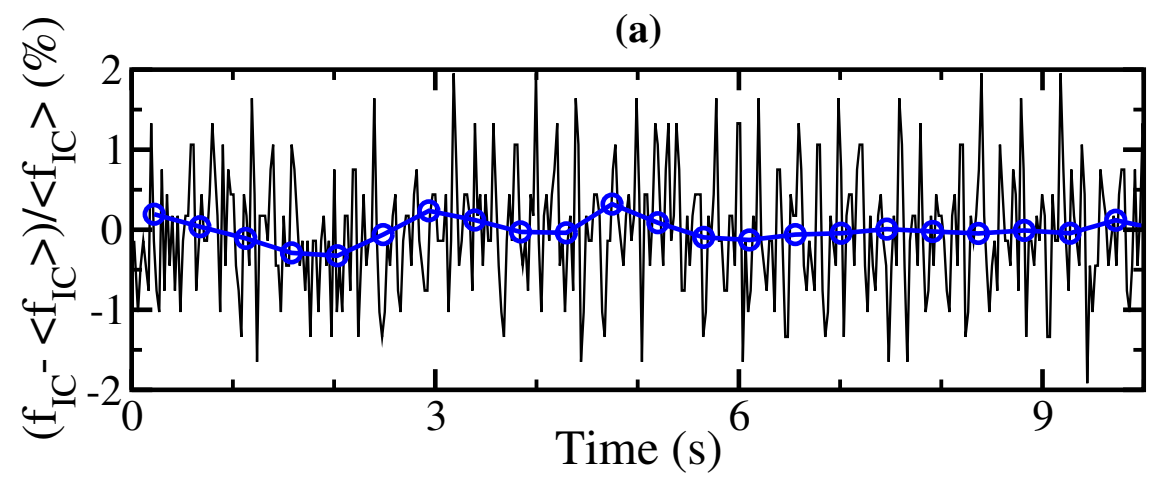

(b)

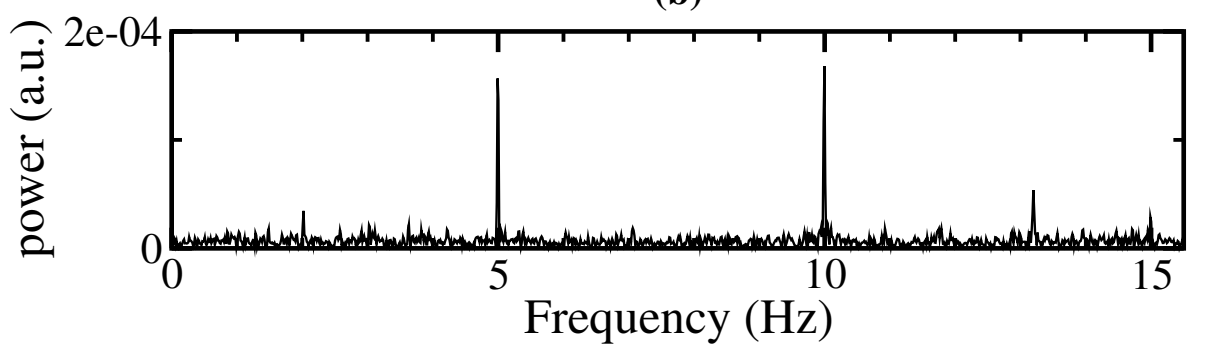

Figure 3.7: (a) Fluctuations of the speed of the motor rotating at $5 \mathrm{~Hz}$. The black line indicates the measured raw data deviation from the mean. The sampling rate is $38 \mathrm{~Hz}$, which corresponds here to 17 measurements per cylinder rotation. The averaged speed value of one cylinder rotation is plotted as blue circle and has a standard deviation of $0.13 \%$. (b) In the spectrum of the raw data signal the motor frequency and its first harmonic are dominating, which is partly due to a systematic deviation of the resolver (see text for further details). The rotation frequency of the inner cylinder $(2.25 \mathrm{~Hz})$ is at noise level.

is carried out with a precision of 65536 ( $2^{16}$ bits) increments per rotation corresponding to 65536 positions of the motor shaft. The positions have geometric deviations that are repeated with each turnover of the motor shaft. As a consequence the motor frequency (and its harmonics) typically appear in the spectrum of the speeds, which is plotted in Fig. 3.7(b). The rotation frequency of the cylinder $(2.25 \mathrm{~Hz})$ is at the noise level in the spectrum, which reflects the high quality of the bearings and the balancing. At double the speed the raw data fluctuations shown in Fig. 3.8(a) decrease slightly to 1\%, the standard deviation of the speed averaged over a cylinder rotation (blue circles) is $0.10 \%$.

Our speed data (Fig. 3.8(a)) are compared to the data from van Gils et al. (2011a) in Twente (Fig. 3.8(b)) for a motor frequency of $10 \mathrm{~Hz}$, corresponding to an inner cylinder frequency of $5 \mathrm{~Hz}$, given their 2:1 pulley ratio. The solid black line in Fig. 3.8(b) corresponds to the raw data, acquired at a rate of $1 \mathrm{kHz}$, underlying the averaged values presented in figure 4 of van Gils et al. The data has been kindly provided by the authors of van Gils et al. (2011a). The reason for the difference between the data in their figure 4 and the averaged data (blue circles) in our Fig. 3.8(b) can be attributed to a different averaging technique and window width. The blue circles in Fig. 3.8(b) are obtained by averaging the raw data over one cylinder turnover (corresponding to $0.2 \mathrm{sec}$ ), whereas van 
(a)

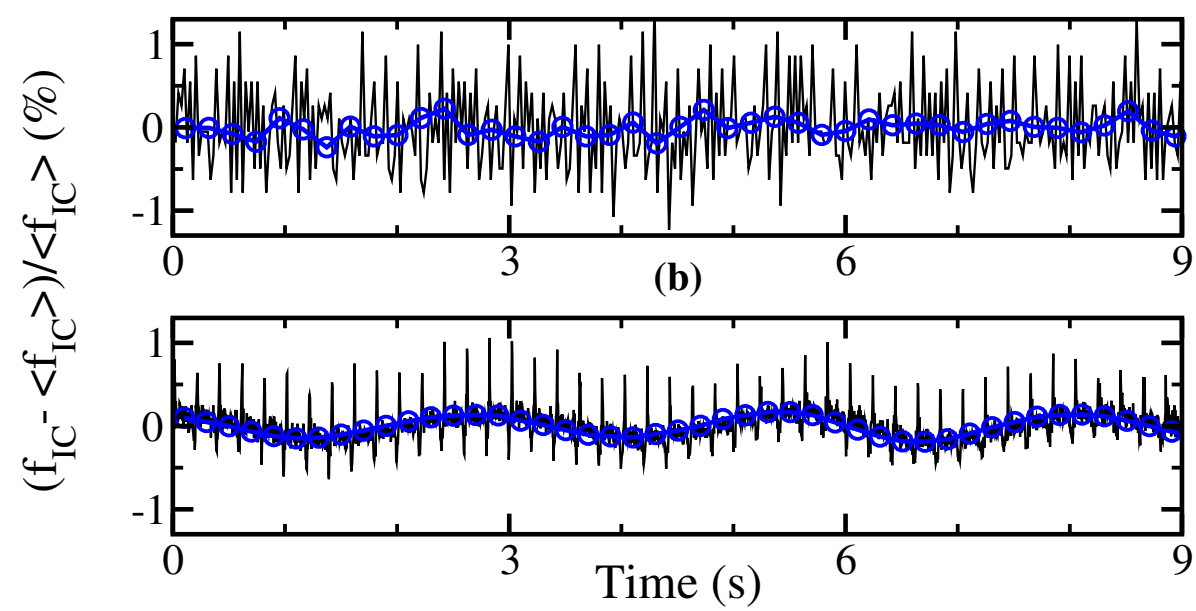

(c)

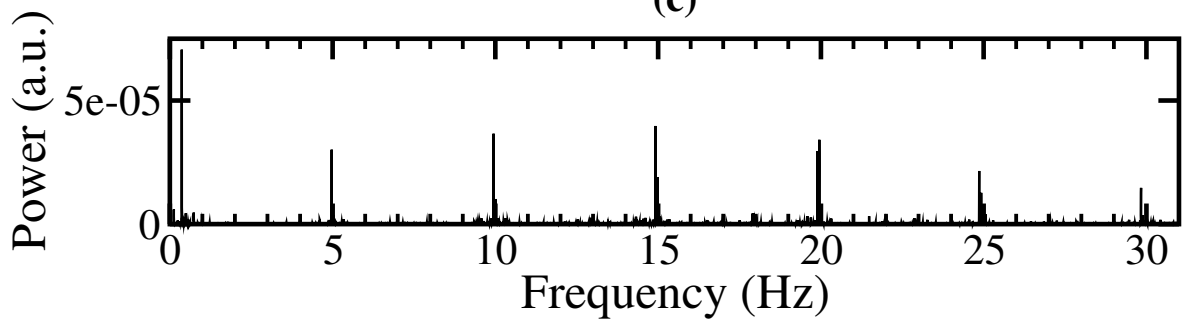

Figure 3.8: (a) Fluctuations of the speed of the motor rotating at $10 \mathrm{~Hz}$ to compare directly to the raw data measurements of van Gils et al.(van Gils et al. 2011a) in Twente, shown in (b). The standard deviation of the speed averaged over a cylinder rotation (blue circles) is in both experiments $0.10 \%$. The main deviation in the Twente experiment is caused by a slow oscillation of $0.37 \mathrm{~Hz}$, which can also be seen in the spectrum of the (Twente) raw data in (c). The peak at $5 \mathrm{~Hz}$ corresponds to the rotation frequency of the inner cylinder, the peak at $10 \mathrm{~Hz}$ to both, the first harmonic of the cylinder frequency and the motor frequency. Their contributions cannot be distinguished due to a gear ratio of $2: 1$.

Gils et al. presented their data by applying a low-pass filter followed by a downsampling resulting in an effective data rate of $1 \mathrm{~Hz}$. In our experiment fluctuations are dominated by the motor frequency. In the Twente experiment an additional slow oscillation of 0.73 $\mathrm{Hz}$ is present, corresponding to a period of almost 7 cylinder turnovers. The authors of van Gils et al. (2011a) would like to point out that they were unaware of the slow and small amplitude oscillation at the time of publication. The standard deviation of the speed averaged over a cylinder rotation is for both experiments the same $(0.10 \%)$. Note that the corresponding $R e_{i}$ and the standard deviation averaged over a cylinder rotation in both experiments are very different with about $1600 \pm 1.6$ in our experiment and $500000 \pm 500$ in Twente. The Reynolds number is calculated as $R e_{i}=2 \pi f_{I C} r_{i} d / v$ with the viscosity $v$. 


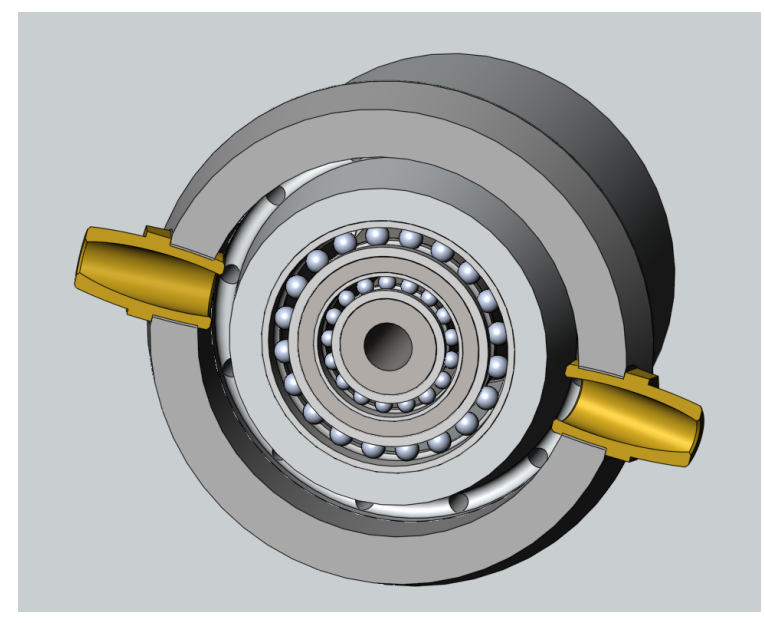

Figure 3.9: A channel system for the cooling liquid surrounds the bearing and seals in the bottom bearing unit. It consists of two horizontal annular channels that are connected by 16 vertical channels. This view from the bottom displays two ball bearings and the bottom horizontal channel with some of the vertical channels.

\subsubsection{Temperature control}

The viscosity of a fluid varies with temperature. Hence a precise Reynolds number control requires a precise temperature control. In the transitional Reynolds number regime it is important to avoid any axial gradient and to minimize the influence of fluctuations of the room temperature on the working fluid. In the turbulent regime it is necessary to remove a large amount of heat. Therefore three parts of the experiment can be temperature controlled, the outer cylinder, the seals and ball bearings and the inner cylinder.

The outer cylinder is surrounded at the sides by a square acrylic box with a wall thickness of $20 \mathrm{~mm}$ and at top and bottom by aluminum plates of $30 \mathrm{~mm}(35 \mathrm{~mm})$ thickness (see Fig. 3.1). The bottom plate has two hose connections with a diameter of $20 \mathrm{~mm}$ at opposite sides through which cooling liquid is entering. It is exiting the acrylic box at the top through 2 hose connections of diameter $32 \mathrm{~mm}$. The large diameters of the hose connectors allow to pump a large amount of cooling fluid through the box.

The seals and the ball bearings can be cooled locally. Therefore the bottom bearing unit includes a cooling channel system consisting of two horizontal (slightly tilted) channels (around the ball bearings and the seals) that are connected by 16 vertical channels with a diameter of $10 \mathrm{~mm}$ (see Fig. 3.9). The cooling liquid is entering at the bottom horizontal channel from where it is streaming upwards along the vertical channels. From there it can either exit into the acrylic box and mix with the other cooling circuit or it can be directly removed in the top horizontal channel thereby generating a separate cooling circuit for the bearings. Note that cooling is not necessary for the bearing at the top of the experiment, since the bearing is frictionless in the first assembly group (air bearings at the outer cylinder) and the working fluid is thermally completely decoupled from the 2nd and 3rd assembly group (bearing supports mounted on the heavy-lift profile). 
In case large amount of heat has to be removed, the setup offers as a third method the possibility to cool the inner cylinder from the inside. In this case the solid drive axle of the inner cylinder is replaced by a hollow shaft that consists of an inner tube (feed) and an annular passage (drain) to create a cooling circuit. On top of the solid bearing support the hollow shaft is connected to a rotary union to allow the coolant liquid to be passed from the stationary lab to the rotating inner cylinder.

For the current measurements in the transitional regime a $0.8 \mathrm{~kW}$ (cooling at $20^{\circ} \mathrm{C}$ ) temperature bath (Lauda Proline RP 845 C) with a pump capacity of $25 \mathrm{l} / \mathrm{min}$ and a suction capacity of $23 \mathrm{l} / \mathrm{min}$ is used. The listed temperature stability is within $0.1 \mathrm{~K}$, measurements of PT100 sensors placed in the cooling liquid at different positions in the experiment (one at the bottom of the acrylic box, one at the top and another one at the horizontal bottom channel) indicate even a relative accuracy of $\pm 0.01 \mathrm{~K}$, which corresponds to the accuracy of the PT100.

\subsubsection{Other (not yet implemented) possibilities}

The experiment offers many new possibilities. Four of them will be explained here that can be achieved with very little time and effort. The first possibility is to investigate the influence of special surface materials and structures (e.g. hydrophobic, nano-structures) on the flow. Instabilities that are exclusively triggered by the endplates and reflection symmetric over the equator (Avila et al. 2008) are perfectly suited for this purpose. The top endplate (or also the bottom endplate) can be exchanged by one with the desired surface properties. The effect of the surface properties can then be easily quantified by the (possible) shift of the onset to higher Reynolds numbers.

The second possibility is to remove the inner cylinder completely and manufacture a new set of endplates. In this case the TC experiment becomes a Von-Karman experiment, the flow between two independently rotating disks. The distance between the disks can be continuously changed during measurements.

The third possibility is to generate a radial temperature gradient to study baroclinic instabilities in quasi-Keplerian flows (Klahr \& Bodenheimer 2003). Instead of cooling the inner cylinder from the inside (see Section 3.3.6) the temperature can be set to a desired value. Additional temperature sensors and the exchange of the solid drive axle of the inner cylinder by a hollow shaft are necessary.

The scaling of torque for independently rotating cylinders (van Gils et al. 2011b, Paoletti \& Lathrop 2011) and different radius ratio (Merbold et al. 2013, Brauckmann \& Eckhardt 2013) has attracted much interest in the past years. In the experiments the method developed by (Lathrop et al. 1992) to measure the torque only at the bulk of the inner cylinder is used and the endplates are either attached to the outer cylinder or kept stationary. In our experiment the aspect ratio and speed of the endplates can be dynamically changed, allowing for a systematic study of geometry and endplate configuration on the torque. Hence the fourth possibility is to measure the torque on the inner cylinder. Here the torque can be measured over the full length of the inner cylinder because of low-friction ball bearings which guide the cylinder in the absence of rotary shaft seals. A commercial torque sensor must be placed between the top of the inner cylinder and the solid drive axle. 


\subsection{Flow visualization}

In this section we outline how we visualize and analyze the flow in the narrow gap of 2.28 $\mathrm{mm}(\eta=0.98)$, without introducing perturbations that trigger subcritical turbulence.

\subsubsection{Working fluid and tracers}

The working fluid is silicone oil with a viscosity of $5 \mathrm{cSt}$, which is seeded with highly reflective aluminum platelets (Eckart, Effect Pigments, STAPA WM Chromal V/80 Aluminum). The concentration of the tracers is less than $1 \%$ in weight (and volume). Due to the elliptical shape the tracers align with the flow and depending on their position they reflect the light, thereby revealing the flow behavior. Note that large dirt particles or clusters of tracers are able to trigger turbulence in the subcritical regime. These disturbances create typically a wake in the downstream laminar flow. When the $R e$ is increased above the subcritical bifurcation, turbulence is immediately triggered spreading in a v-shape from this particle. The spreading rates have been analyzed quantitatively for a controlled perturbation (Hegseth et al. 1989, Burin \& Czarnocki 2012). Great care has to be taken to remove this kind of perturbations for measurements in the subcritical regime.

\subsubsection{Spatio-temporal analysis}

The goal of the spatio-temporal analysis is to determine the turbulent fraction of the flow for given Reynolds numbers. The illumination is therefore optimized to distinguish between laminar and turbulent flow patches. By illuminating the flow (seeded with tracers) only from the top by two light-emitting diods (LED) lamps (each consisting of 96 LEDs, 400 lumen) and monitoring the flow at $90^{\circ}$ angle, the light intensity is related to the turbulence intensity. In laminar flows the tracers align all in a similar direction. With the current position of the illumination and the camera only scattered light is detected. This corresponds to the minimal light intensity. In the turbulent patch the tracers are randomly oriented, so that some tracers (e.g. flat surface in an 45 degree angle between light and camera) reflect the light directly to the camera causing peaks in the light intensity. Since the light intensity never drops significantly below the value of the laminar flow (because scattered light is always present), the turbulence leads in average to a net increase in the detected light intensity.

The flow is monitored with a 4 Megapixel high-speed CCD camera (Phantom V10), and typical sampling rates used here are $80 \mathrm{~Hz}$. The spatio-temporal dynamics is simply retrieved by plotting the light intensity along a line of 3 pixels width parallel to the cylinder (marked as green line in Fig. 3.10(a)) from each frame over time. Three steps of analyses are necessary to retrieve the turbulent fraction and are explained in the following.

In the first step the background has to be removed. The raw data profile of the light intensity from the snapshot in (a) showing subcritical turbulence is plotted in green in (b). The peaks corresponding to the turbulent spiral arm are clearly visible. The black line corresponds to the axial intensity profile of purely laminar flow and is used for the analysis of all images. It is determined by averaging the light intensity of laminar flow along the vertical line over about 10 seconds. The profile reflects the non-uniform illumination 
(a)

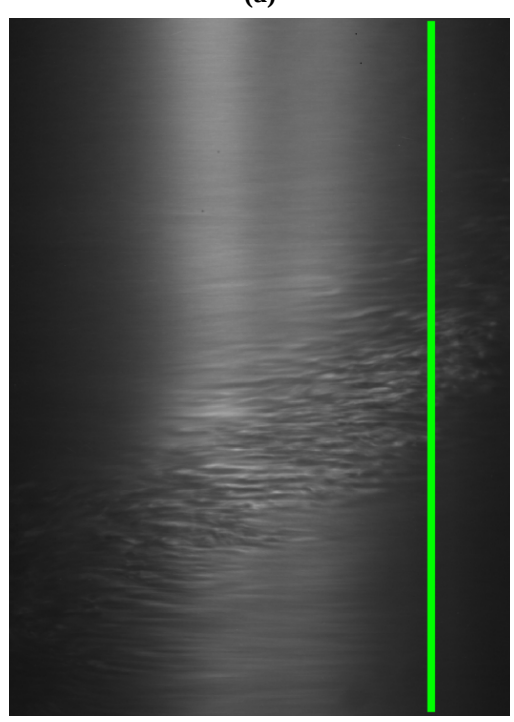

(b)

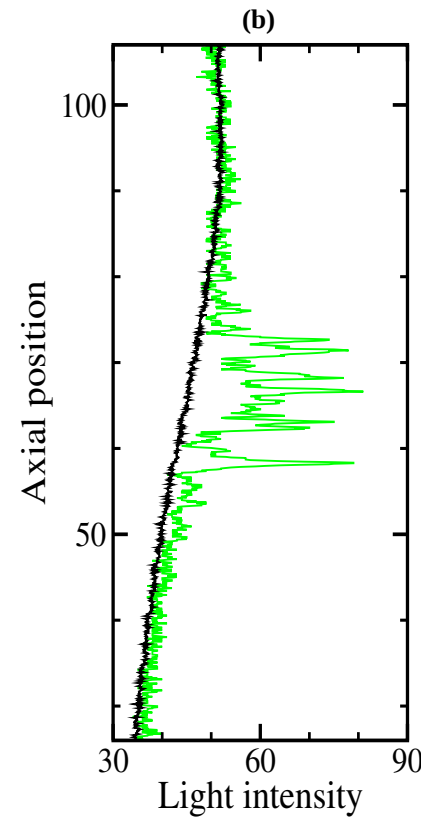

(c)

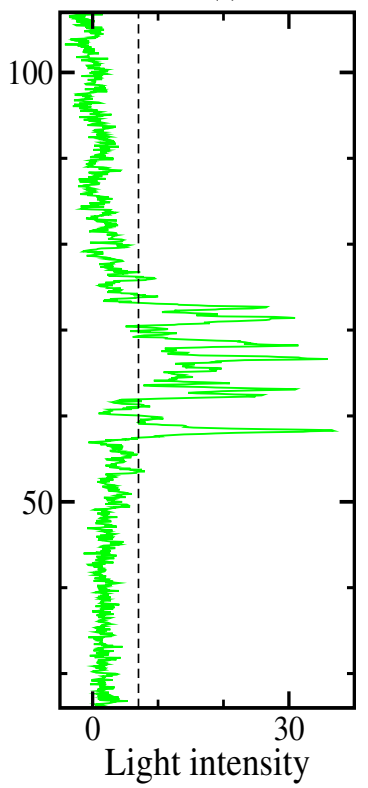

Figure 3.10: (a) Snapshot of a subcritical turbulent spiral arm by a high-speed CCD camera. The raw data profile of the light intensity along the green line is plotted in (b) as a green line, the black line corresponds to laminar flow. (c) Profile of the light intensity after subtracting the laminar flow. The peaks indicate clearly the location of the turbulent spiral arm. For the further analysis a light intensity value above the dashed line is counted as turbulent flow, a value below as laminar flow. Note that the illumination is optimized for the image processing and not for the observation by eye.

along the axial direction (background). This effect is compensated by subtracting this (laminar) profile from the turbulent raw data signal, as it is shown in (c).

In the second step the analysis distinguishes between laminar and turbulent regions by setting a threshold that is clearly above the noise level of the laminar flow, displayed as black dashed line in Fig. 3.10(c). Even though the choice of this threshold will slightly affect the absolute value of the turbulent fraction, the scaling behavior is likely not affected by it and this will be checked in later studies. More important is that a single threshold can be applied along the full length of the monitoring window. While the background can be simply subtracted from the images, the height of the peaks corresponding to turbulent flow has to be rather equal within fluctuations. This is determined by the illumination (exact position of the LEDs) and is the limiting factor to increase the window length.

By plotting the profiles like in Fig. 3.10(c) from all frames after each other, the space-time diagram in Fig. 3.11(a) is generated. The time is given in advective units $\left(d /\left(2 \pi\left(f_{I C} r_{i}-f_{O C} r_{o}\right)\right)\right.$ with $f_{O C}<0$ for counter-rotation $)$. Lighter regions correspond to turbulent flow, dark regions are laminar. The random orientation of the tracers in the turbulent flow includes also orientations typical for the laminar flow. These may lead locally and temporally confined to a drop of the light intensity below the threshold. In the binarized space-time diagram (after thresholding) they appear as tiny black holes surrounded by white (turbulent) regions. In the third and last step of the image processing these black 
(a)

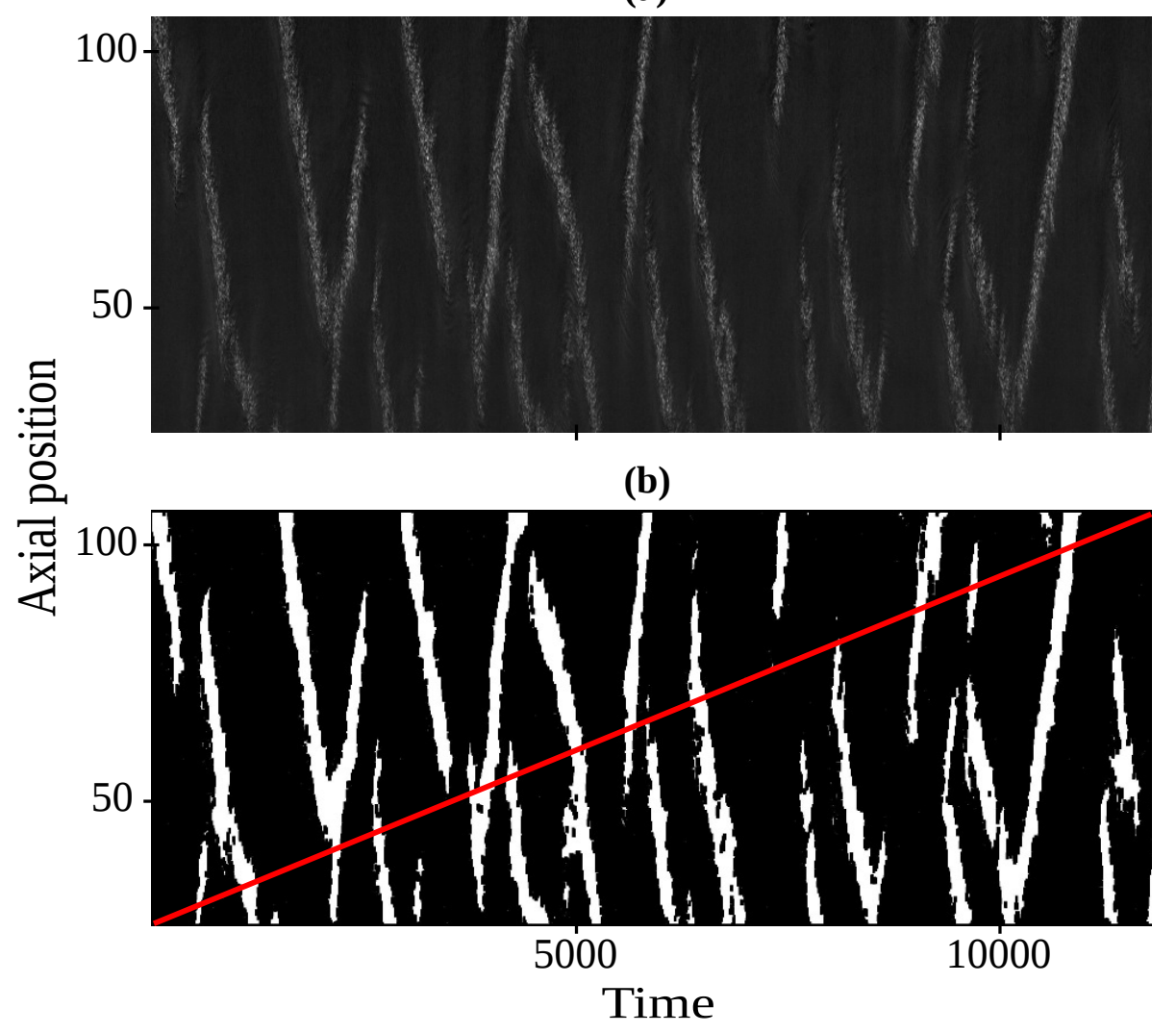

Figure 3.11: Space-time diagram from a flow visualization at successive steps of the image processing to determine the turbulent fraction: (a) Image of the light intensity along the green line in Fig. 3.10(a) with time after subtracting the laminar flow. Lighter regions indicate turbulent flow, dark regions are laminar. (b) Binarized image after thresholding, eroding and dilating. The turbulent fraction corresponds to the ratio of the white (turbulent) regions over the total area. The profile along the red line is plotted in Fig. 3.12.

holes are eliminated by first dilating and afterwards eroding the image with the same parameters (e.g 19 pixels in the axial direction, 7 pixels in the time-direction). The resulting spatio-temporal diagram is displayed in Fig. 3.11(b). The turbulent fraction corresponds to the ratio of the white (turbulent) regions over the total area.

To allow for a direct comparison between the raw data signal (without background) and the final result of the image processing, the profile along the red line in Fig. 3.11(b) is plotted in Fig. 3.12(a) and partly magnified in (b). The black solid line corresponds to the raw data signal and the black dashed line indicates the threshold to distinguish between laminar and turbulent flow. Note that the peaks corresponding to turbulent flow have on average a comparable height and can be clearly distinguished. The red line is the final result of the image processing. A value of 20 (here arbitrary set for demonstration) represents a turbulent patch and a value of zero a laminar one. 
(a)

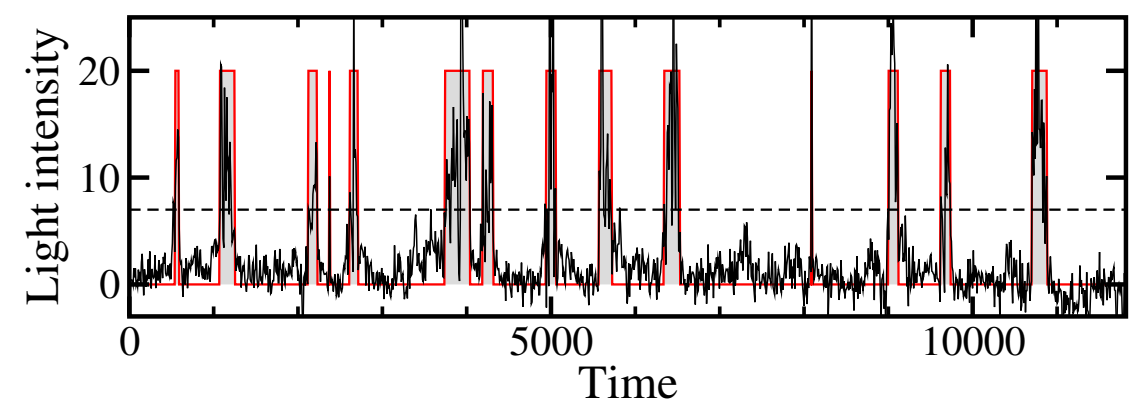

(b)

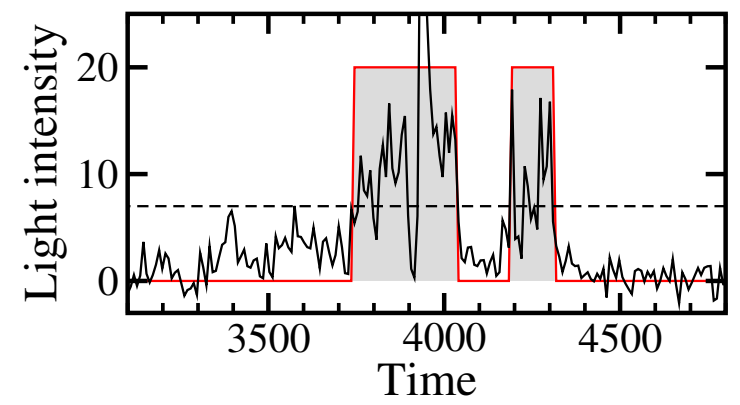

Figure 3.12: (a) Comparison of the raw data signal (without background) plotted as black solid line with the result of the image processing (red line) for a profile through space and time along the red line in Fig. 3.11(b). A value of 20 in the final result (here arbitrary set for demonstration) represents a turbulent patch, a value of zero a laminar one. (b) Magnification of (a)

\subsection{Examples of results}

\subsubsection{Onset of Taylor vortices for stationary outer cylinder $(\eta=0.98)$}

The primary instability for a radius-ratio of 0.98 with a stationary outer cylinder is the Taylor vortex flow (TVF). In a finite system Ekman vortices form close to the endplates and excite Taylor vortices in their vicinity, so that the onset of Taylor vortices is not a bifurcation (Benjamin 1978). However, by flow visualization it can be easily seen when Taylor vortices occur in the bulk of the system, which is considered as onset of the TVF (Carey et al. 2007). The onset can be determined by linear stability theory to $R e_{i}=291.6$ for $\eta=0.980$, which we use to determine the viscosity of the working fluid. Especially in high-radius ratio experiments the onset of TVF can vary locally up to a difference in Reynolds number of more than 80 (Carey et al. 2007) most likely due to deviations of the gap width. In our experiment the onset of the TVF is uniform across the bulk $(<1$ $R e_{i}$ corresponding to deviations $<1 \%$ ), reflecting the high quality of the bearing and the cylinders. This is remarkable since the gap width has local deviations of up to $3 \%$, but it seems that the flow averages this out. 


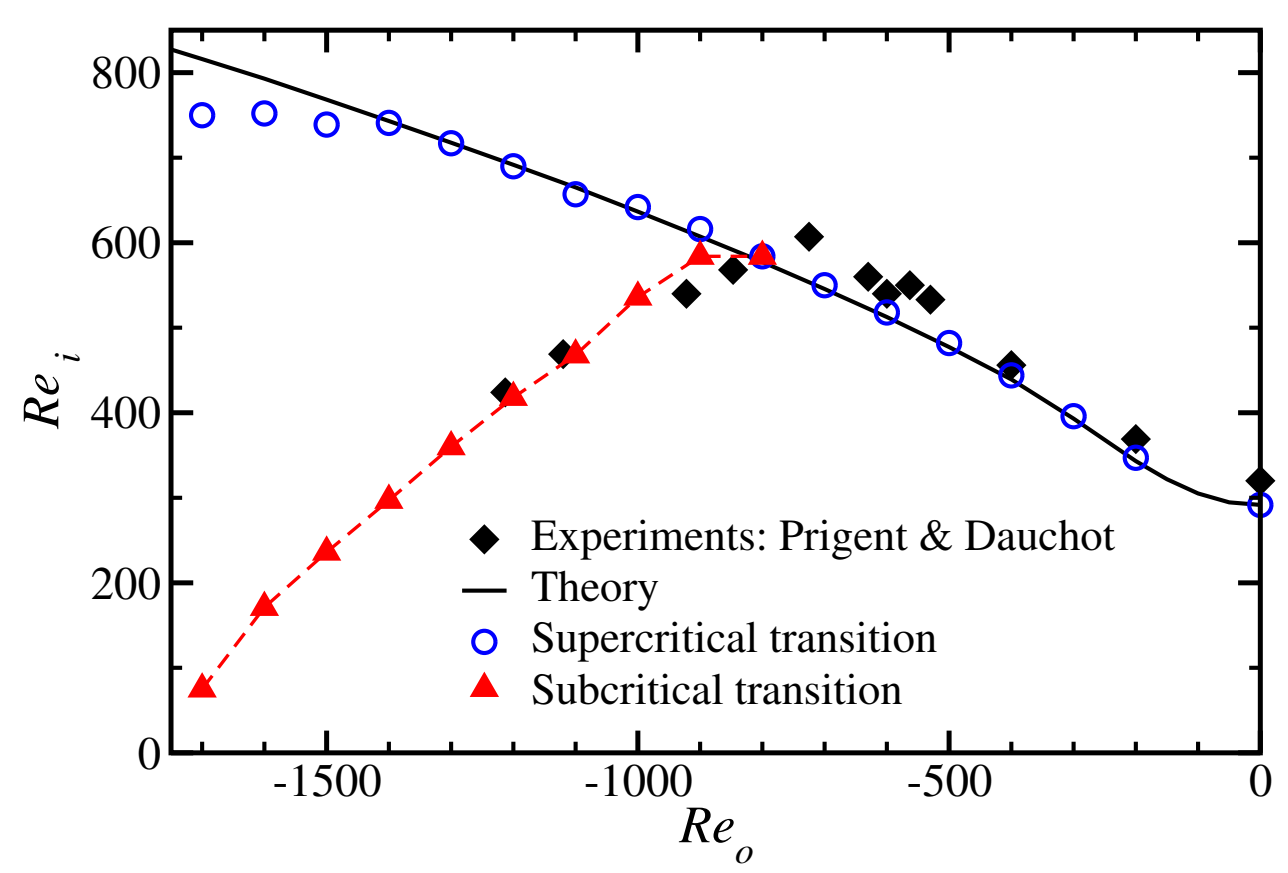

Figure 3.13: Stability diagram for $\eta=0.98$. For increasing $R e_{i}$ at a constant $R e_{o}$ the supercritical onset of instabilities (blue circles) agrees well with the linear stability analysis (black line). Subcritical turbulence in the form of turbulent spirals and spots is found for decreasing $R e_{i}$ and constant $R e_{o}<-800$ until it decays approximately at the red triangles. No hysteresis was observed in previous measurements for a similar $\eta$ from Prigent, Dauchot and coworkers (black diamonds) (Prigent et al. 2003, Prigent \& Dauchot $2005,2000 a$ ) due to finite amplitude perturbations triggering turbulence in the subcritical regime. Here finite amplitude perturbations trigger instabilities (blue circle) only slightly below the linear instability for $R e_{o} \leq-1500$.

\subsubsection{Onset of the linear instability for counter-rotating cylinders with $\eta=0.98$}

In the first measurements we investigate the stability of the laminar flow for counterrotating cylinders and stationary endplates. For each measurement the Reynolds number of the outer cylinder $R e_{o}$ is kept constant, while the Reynolds number of the inner cylinder $R e_{i}$ is increased quasi-statically ( $1 R e_{i} / 2 \mathrm{~min}$ ) until an instability sets in (blue circles in Fig. 3.13). The onset of these supercritical instabilities deviates less than $1 \%$ from the predicted values by linear stability analysis (black line in Fig. 3.13 for $R e_{o}>-1500$. On the other hand for $R e_{o} \leq-1500$ the instabilities set in systematically below the values predicted by theory. In this regime finite amplitude perturbations already trigger the transition to turbulence, showing that here turbulence can be triggered subcritically and reflecting how sensitive the system is.

In the following measurements we investigate the transition from turbulent (timedependent modes) to laminar flow for quasi-statically decreasing $R_{i}\left(1 R e_{i} / 1 \mathrm{~min}\right)$. No hysteresis is observed for $R e_{o} \geq-800$, but for higher counter-rotation rates the hysteresis is clearly evident. This subcritical transition border is approximated by the measurement 
method and displayed as red triangles. Note that the decay of turbulence is a stochastic process and the real critical phase transition point can only be determined by analyzing the turbulent fraction, which is one goal of our future studies.

Stability diagrams similar to Fig. 3.13 have been determined for smaller radius-ratio systems (Andereck et al. 1986), but it is the first time in a high-radius ratio experiment $(\eta \geq 0.98)$ that the supercritical transition could be clearly distinguished from the subcritical one. The challenge is to minimize finite amplitude perturbations that would otherwise trigger the transition to turbulence in the linearly stable regime (below the black line in Fig. 3.13). This becomes clear by comparing our measurements to the only existing studies in this regime by Prigent, Dauchot and coworkers for an $\eta=0.983$ (black diamonds in Fig. 3.13)(Prigent et al. 2003, Prigent \& Dauchot 2005, 2000a). For $\operatorname{Re}_{o} \geq-800$ the measurements agree qualitatively with ours, but for higher counter-rotating rates their measurements do not exhibit hysteresis. The transition between laminar and turbulent flow is always at the subcritical border independent of the measurement procedure (in- or decreasing $R e_{i}$ ), triggered by finite amplitude perturbations. This is not of great importance for their investigations in the supercritical regime (Prigent et al. 2002), but in order to determine the equilibrium turbulent fraction and to characterize the phase transition as we are planning here it is absolutely crucial to avoid such uncontrolled finite amplitude disturbances.

\subsection{Discussion}

We have built and tested the first high-radius ratio Taylor-Couette experiment $(\eta=0.98)$, in which perturbations are so small that the phase transition towards sustained turbulence in the subcritical regime can be investigated. The system size is 12 times larger than the largest plane Couette experiment, that can technically still be controlled to study the phase transition (Bottin \& Chaté 1998). In future this together with a measured accuracy of better than $1 \%$ will allow us to measure the turbulent fraction significantly closer to the critical phase transition point than all previous experiments. The optical measurement method and analysis developed, enables the measurement of both the fast dynamic and the critical slowing down, up to time scales of $10^{7}$ advective units, three orders of magnitude longer than previous numerical simulations (Duguet et al. 2010).

By an independent rotation of the axial endplates combined with a variable aspect ratio, this device bridges the gap in astrophysically motivated investigations between large aspect ratio experiments with axial endplates attached to the outer cylinder and small aspect ratio systems with an active control of the axial boundaries. In this experiment it could be tested if endplate driven instabilities at low Reynolds numbers (Avila 2012) are eventually superseded by shear flow turbulence with universal scaling at large $R e$ (Paoletti et al. 2012), as recently suggested (Avila 2012). A clear distinction between these two regimes is necessary before experimental results from the TC system can be used to infer the dynamics of turbulence in accretion disks.

Besides these two specific applications the device features a large number of control parameters allowing to investigate a big variety of different aspects of rotating flows. We only want to point out the large Reynolds number regime in which the experiment can operate $\left(\operatorname{Re}=10^{1}-10^{6}\right)$, the possibly extreme radius-ratios $(\eta=0.03$ to 0.98$)$ and the 
excellent optical access.

\subsection{Acknowledgments}

We acknowledge K. Rühl (arkkon Gmbh, Germany) for his technical and engineering support and particularly his enthusiasm and ideas in finding various technical solutions. We appreciate discussions about motor fluctuations with D. van Gils and the coauthors of (van Gils et al. 2011a) and the sharing of their data. We want to mention the excellent technical service of Novotron (Germany), especially of R. Mühlberger and we thank M. Avila for computing the linear stability (black line in Fig. 3.13). The research is financially supported by the Max Planck society and the Deutsche Forschungsgemeinschaft (FOR 1182). K.A. acknowledges support from the International Max Planck Research School for the Physics of Biological and Complex Systems and the Göttinger Graduate School for Neurosciences, Biophysics and Molecular Biosciences. 



\section{Phase transition in Couette flows}

In the introduction (Section 1.3.3), the results of previous models (Manneville 2009), experiments (Bottin \& Chaté 1998, Bottin et al. 1998) and simulations (Duguet et al. 2010) on the phase transition between decaying and spreading turbulence have been summarized. The flow dynamics and models indicate that directed percolation might be a proper description for this transition. But observations of direct numerical simulations and experiments in plane Couette flow show a behavior that is not in agreement with this expectation. They report the transition to be of first order, instead of second order, which is required for directed percolation. However, a closer look on the scaling of the turbulent fraction (see Fig.1.16) revealed that the simulations are not close enough to the critical transition point to be able to distinguish between a first and a second order phase transition. The problem are the time scales to reach the steady state which increase as the critical point is approached. The experiments provided long observation times, but the system size was very limited. Attempts to build an experiment with a larger system size failed due to an insufficient precision (Prigent et al. 2003).

In this section I will discuss the interpretations of the previous experiments by highlighting the influence of the system size and the accuracy of the setup on this phase transition, before presenting my own measurements. In this context, the linear stability of the laminar flow is very important. If all turbulence in the system has decayed, the flow remains laminar for all times. In the framework of statistical mechanics this behavior is reflected in the non-equilibrium class of phase transitions. Finite amplitude perturbations are necessary to trigger the transition to turbulence. In these investigations such perturbations are applied only once to create turbulence initially. While the mean turbulent fraction is measured, such perturbations have to be avoided to observe the intrinsic dynamics of the flow.

\subsection{Influence of the system size}

In order to investigate a phase transition large statistical ensembles have to be analyzed. It is therefore commonly known that a large system size is required. In most systems the system size in experiments is by default large enough to approximate many characteristics of phase transitions very well. An example is the loss of spontaneous magnetization when the temperature exceeds a critical value, which is described by the Ising model. In a ferrum cube with side length of $1 \mathrm{~cm}$ there are about $10^{9}$ electronic spins (Bramfitt 2001). The net magnetization depending on the temperature in an experiment shows clearly a second order phase transition. As I will show in this section, the limited size in plane Couette experiments might change the appearance of the phase transition. The 
(a)

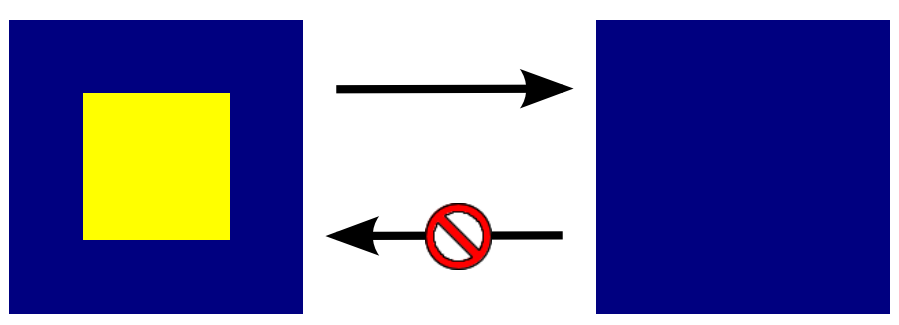

Figure 4.1: (a) Sketch of the influence of the system size on the phase transition. The blue area corresponds to the laminar flow and the yellow area to the minimal turbulent structure that is sustained. In pipe flow this structure corresponds to a puff, in TaylorCouette flow to a single spiral arm and in plane Couette flow to a turbulent band. In this system the minimum turbulent fraction that is still sustained corresponds therefore to $25 \%$. (b) Measurements closer to the critical point (by decreasing $R e$ ) would lead to the decay of the turbulent structure and the the flow would relaminarize. Since the laminar flow is linearly stable, the turbulence cannot recover and the flow remains laminar for all times.

main difference stems from the minimal turbulent structure that can be sustained by the mean flow. In pipe flow, such a structure is a puff, which is localized in the streamwise direction to about 20-30 pipe diameters (Wygnanski et al. 1975). If a puff is 'squeezed' by an external force, e.g. by another puff it will decay (Hof et al. 2010, Samanta et al. 2011). In Taylor-Couette and plane Couette flow turbulence is localized in the form of spirals or tilted bands, respectively. The width of these bands is about 20-40 gap units (Prigent et al. 2002). My observations of flow visualization in high-radius ratio Taylor-Couette flow $(\eta=0.98)$ suggest that a spiral arm has a rather constant width in the streamwise (azimuthal) direction, but its length in the spanwise (axial) direction changes substantially. As long as the turbulence arranges itself in a spiral arm (either confined by the axial lids of the experiment or by an intrinsic localization) it can usually be sustained. But if the turbulence arranges itself in a spot of similar dimensions in streamwise and spanwise direction it usually decays, unless it can recover its spiral shape in a time corresponding to about 5-10 times propagating around the inner cylinder.

To sum up, it seems that in linearly stable shear flows a minimum turbulent structure exists. In contrast to the aforementioned magnet, here these structures have a macroscopic size, which is on the same order as the system size of experiments. If in average exactly a single of this turbulent structures exists in the system, then this defines the minimum turbulent fraction that can be measured in a setup. In the example in Fig. 4.1(a) this minimum turbulent fraction corresponds to $25 \%$. If the system size increases by 10 , the minimum turbulent fraction that can be measured is 10 times smaller, namely $2.5 \%$. In this example it is therefore the size of the system and not the dynamics of the flow that determines the size of the discontinuity. The classification of a transition in first and second order on the other hand depends exactly on the size of this discontinuity. Applied to experiments it means that this phase transition will always appear discontinuous, even though it might be of second order.

The minimum turbulent fraction in the plane Couette experiments was about $35 \%$ 


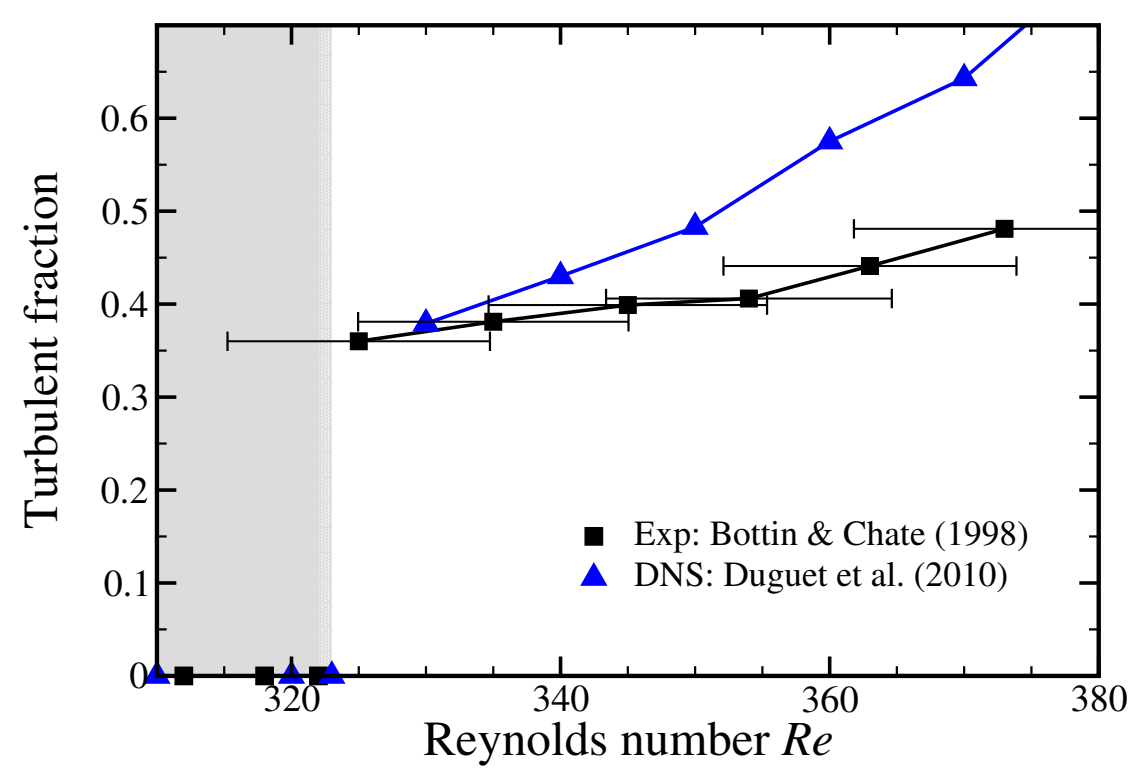

Figure 4.2: Previous experimental (Bottin \& Chaté (1998), Bottin et al. (1998)) and numerical (Duguet et al. (2010)) investigations in plane Couette flow interpreted the phase transition to be of first order. The minimum turbulent fraction that was sustained is above $35 \%$. The gray area indicates where the turbulence was observed to decay. It is clearly visible that the error bars with about $3 \%$ of the $R e$, stemming from the experimental precision reach inside this regime. The consequences on the nature of the transition are discussed in Fig. 4.3.

(Bottin \& Chate 1998). If the transition is of first or of second order could be determined by increasing the system size and checking if the minimum turbulent fraction decreases. An almost ten times larger plane Couette experiment was indeed build, but the accuracy in $R e$ was with about $7.5 \%$ not sufficient to investigate this phase transition (Prigent et al. 2003). In the following it is demonstrated why the accuracy is so important.

\subsection{Influence of the accuracy of the experimental setup}

So far we have assumed that an experimental setup has a finite size but no uncertainties, which is obviously wrong. In the plane Couette experiments of Bottin et al. (1998) the accuracy in $R e$ is estimated to about 3\%, which I plotted as error bars in Fig. 4.2. It can be clearly seen that the error bars reach inside the gray shaded area, which indicates the transient turbulent regime. The influence of deviations in $R e$ on the flow dynamics close to this regime is qualitatively different from its influence at larger turbulent fractions.

This becomes clear by looking at the simple example in Fig. 4.3. We assume that $R e$ drops temporally or locally due to an inaccuracy of the experiment, e.g. stemming from a temperature drop or a varying gap width. If several turbulent patches are present in the system like in Fig. 4.3(a) this drop in $R e$ leads to the decay of several patches. If $R e$ increases afterwards, new patches will be generated from existing ones by processes similar to puff splitting in pipe flow. If the deviations in $R e$ are symmetric to higher 
(a)

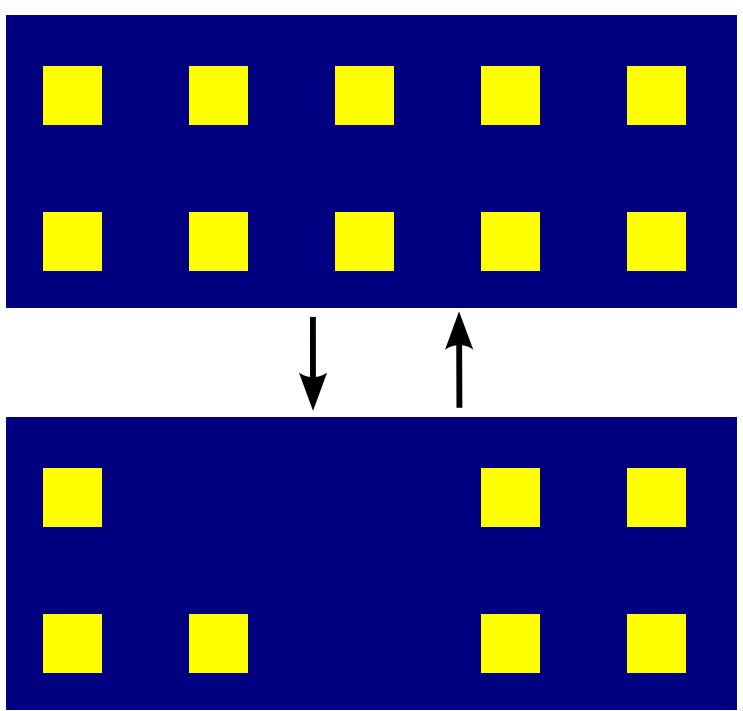

(b)
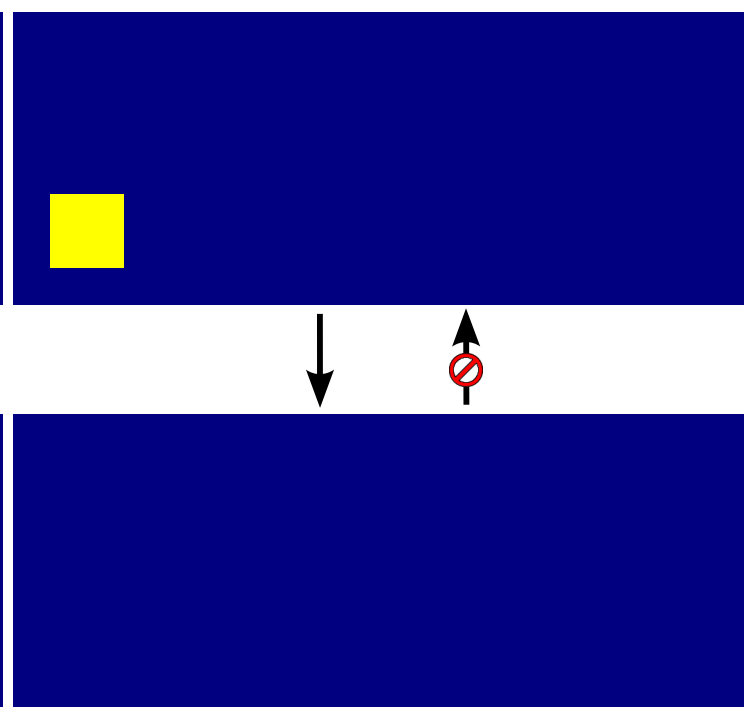

Figure 4.3: (a) In this large system 10 turbulent patches (marked in yellow) correspond to a turbulent fraction of $25 \%$. If $R e$ is temporarily decreased, either on purpose or due to an imprecision of the experiment, some of the patches decay. If $R e$ increases again, the turbulent patches spread, thereby generating new ones. The turbulent fraction remains constant apart from fluctuations around the mean. (b) Close to the minimum turbulent fraction (here corresponding to $2.5 \%$ ), a decrease in $R e$ will lead to the relaminarization of the flow. If the $R e$ is increased afterwards, the flow remains laminar.

and lower values, these deviations will lead to a fluctuation around the mean turbulent fraction. By using long observation times precise measurements are possible even though the experiment itself might not be that accurate.

The situation is completely different close to the minimum turbulent fraction of the setup as it is displayed in Fig. 4.3(b). In average only a single turbulent patch will be present. A drop in $R e$ is likely to kill this last turbulence. Once this patch has decayed the flow remains laminar, even when $R e$ increases again. Deviations in $R e$ close to the minimum turbulent fraction are therefore not a fluctuation around the mean value, but influence how the phase transition appears.

\subsection{Phase transition in a large system with high accuracy}

If we assume that the onset of turbulence follows a second order phase transition then it is the system size that determines the minimum turbulent fraction that could be possibly measured. The accuracy of the setup determines which turbulent fraction is sustained and can actually be measured. System size and accuracy of the experiment are therefore the key players that control how close to the critical point turbulence is sustained. They determine how well a continuous scaling can be approximated.

In this thesis the measurements of the phase transition are performed in a high-radius ratio Taylor-Couette system (radius ratio $\eta=0.98$ ), whose system size is 12 times larger in 


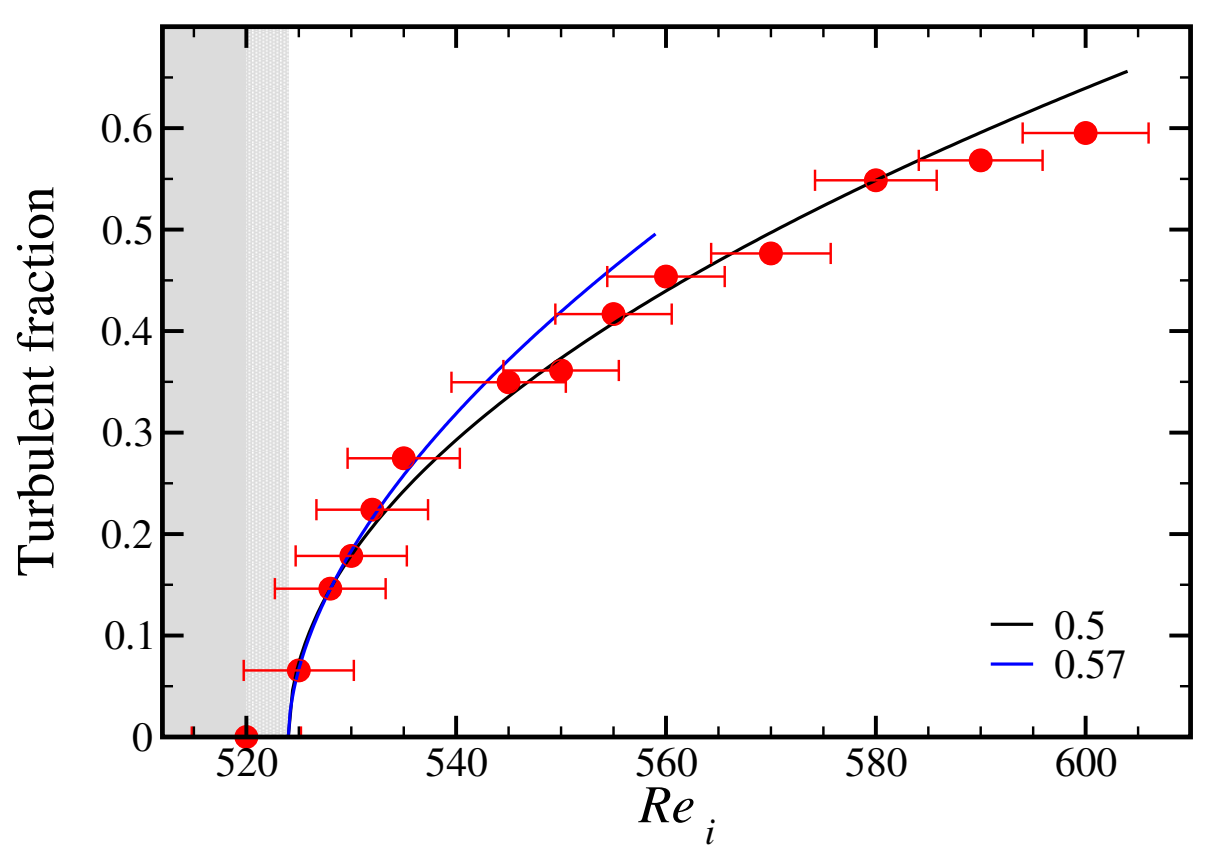

Figure 4.4: Phase transition of second order in a high radius-ratio Taylor-Couette experiment $(\eta=0.98)$. The minimum turbulent fraction corresponds to about $7 \%$. The system size is 12 times larger and the observation time 30\% longer than in Bottin \& Chaté (1998). The error bars were determined by comparing the onset of linear instabilities with linear stability analysis and correspond to $1 \%$ of the $R e$. The blue line is a power law fit to the data points in the vicinity of the critical point $\left(520<R e_{i}<550\right)$. The corresponding exponent of $\approx 0.57$ agrees well with the one from 2-dimensional directed percolation (0.58). A square-root scaling is shown by the black line for comparison. During the measurements $R e_{o}=-1000$ and the endplates are at rest. The linear stability sets in at $R e_{i}=637$.

$\operatorname{area}^{1}$ than the previous plane Couette experiment of Bottin \& Chaté (1998). The accuracy in $R e$ is about $1 \%$, which was estimated by comparing the onset of linear instabilities with linear stability analysis for a pure inner cylinder rotation and counter-rotating cylinders (see Fig. 3.13 on page 81). Finite amplitude perturbations are better controlled than in previous Taylor-Couette experiments with the same $\eta$ (see also Fig. 3.13). The turbulence was triggered by increasing $R e_{i}$ until the flow becomes linearly unstable. Afterwards $R e_{i}$ was abruptly decreased to the desired value and the measurements started after the flow reached a steady state. The turbulent fraction is obtained in the counter-rotating regime, where the laminar profile is linearly stable (subcritical turbulence). The dynamics resembles here the one of pipe and plane Couette flows (Coles 1965, Prigent et al. 2002, Borrero-Echeverry et al. 2010). The outer cylinder speed is kept constant throughout the study at $R e_{o}=-1000$ and the endplates confine the axial length to 263 gap width units and are at rest. The turbulent fraction is determined by analyzing the images from a high speed camera (see Section 3.4.2), which monitors the flow after it has reached its steady state. The recording time reaches from 90 seconds at the largest $R e_{i}$ to 15 minutes at the

\footnotetext{
${ }^{1}$ The spanwise length is about 7.5 times larger and the streamwise about 1.6.
} 
lower $R e_{i}$ corresponding to $1.4 \cdot 10^{6}$ advective units. The recording times increase as the critical point is approached to account for the critical slowing down.

The retrieved turbulent fractions are plotted in Fig. 4.4. It is clearly visible that the minimum turbulent fraction is substantially smaller than in the plane Couette experiments of Bottin \& Chaté (1998). In fact it is five times smaller and the observation time is about $30 \%$ longer than in the plane Couette experiments. The turbulent fraction increases smoothly with increasing $R e_{i}$ from its minimum value of about $7 \%$ to more than $50 \%$. From these results it can be concluded that the phase transition is likely to be of second order.

In these measurements the scaling of the mean turbulent fraction can be approximated by a square-root over the full $R e_{i}$-regime, which is indicated by the black line. It seems that closer to the critical point an exponent of 0.57 , shown by the blue line, approximates the measurements better. This exponent agrees well with the one from two dimensional directed percolation $(0.58)$.

Please note that these measurements are preliminary. I do believe that the phase transition is of second order, a second set of preliminary experiments (performed by Dr. Jalikop and not shown in this thesis) has confirmed this. But even though the scaling of the turbulent fraction is exactly what we were hoping for, namely a scaling corresponding to directed percolation, this has to be checked in a new set of experiments. In these measurements the long-time condition of the working fluid is problematic. Usually the fluid volume in the gap is used throughout the whole study and the experiment is working 24 hours a day to minimize any change of the conditions. This was not possible for these measurements, because the working fluid was leaking out of the experiment due to a worn out seal. Several times new working fluid was added. Even though the fluid comes from the same container, its temperature might not have been exactly the same (deviation $\geq 0.01 K$ ) and particle sizes/concentration of the tracers might have slightly changed. In addition, refilling the experiment trapped air bubbles. These were removed, but overall it remains to be said that the conditions were not as good as I would have liked them to be. Future experiments are already in preparation with measurements closer to the critical point and even longer observation times. By analyzing also the distribution of the laminar gaps to obtain the second and third exponent, these measurements will finally clarify if the onset of turbulence can be described by directed percolation or at least if it can be observed in an experiment at all. 


\section{Discussion}

The transition to turbulence has been and continues to puzzle researchers since the pioneering experiments in pipe flow of Reynolds (1883) in the 19th century. Although the onset of turbulence in flows that become linearly unstable is well understood since the work of Sir Taylor (1923) and Ruelle \& Takens (1971), the transition in linearly stable laminar flows has remained quite mysterious. For the latter there does not exist a theory that can either predict the exact transitional Reynolds number $R e$ or even describe the nature of the transition route qualitatively. Even one of the simplest questions, at what critical $R e$ pipe flow remains turbulent, was unanswered since the work of Reynolds (see e.g. Eckhardt (2008)).

In this thesis (Chapter 2) the question has been answered experimentally. The critical $R e_{c}$ is $2040 \pm 10$. But much more important than the exact value is the knowledge gained by determining this number. Prior to this work it was suggested that the nature of turbulence in pipe flow is transient (Hof et al. 2006). In fact, the decay of turbulent puffs is a stochastic, memoryless process (Faisst \& Eckhardt 2004), and the time scales involved grow explosively with increasing $R e$, but remain always finite (Hof et al. 2008, Avila et al. 2010, Kuik et al. 2010). Therefore, from these lifetime studies of single puffs no critical $R e_{c}$ could be determined. However, during my thesis Moxey \& Barkley (2010) compared the onset of puff splitting, through which turbulence can spread, with the time scales of the decay. They concluded that even though the nature of a single turbulent puff might be transient, the mechanism of splitting can fill the pipe with intermittent turbulence. The main drawback of their work was that they assumed puff splitting to be a deterministic mechanism starting at a critical $R e \geq 2300$. Moreover, they postulated that the onset of puff splitting is equal to the onset of sustained turbulence. At that time I was in the laboratory performing a statistical investigation of the puff splitting mechanism. The results of Chapter 2 show that puff splitting is not a deterministic mechanism, but rather a stochastic, memoryless process, exactly like the lifetime dynamics. By combining this knowledge with the idea of Moxey \& Barkley of comparing splitting and decay it was possible to determine in this thesis the 'real critical value' (Reynolds 1883) for the onset of turbulence in pipe flow.

The measurements in Chapter 2 show that the mean time of the splitting increases superexponentially for decreasing $R e$. The mean lifetimes, on the other hand, increase superexponentially for increasing $R e$ (Hof et al. 2008). At the critical $R e_{c}=2040$ the time scales of both processes balance each other. For smaller $R e$ the mean time of decay is shorter than the mean time of splitting. This means in average the turbulence will decay and the flow relaminarize. For larger $R e$ the opposite situation occurs: the mean time of the decay is longer than the one of the splitting and hence in average a puff will split more 
often than decay. In this case the pipe fills with intermittent turbulence. Note, that an individual puff at $R e>2040$ could possibly decay before it were able to generate another puff via puff splitting. In this case, the flow would reverse to its laminar state. Only in an infinitely long pipe, which has infinitely many puffs traveling downstream the concept of the critical $R e_{c}$ is a valid description.

These arguments bring us to statistical mechanics, and indeed this transition can be interpreted as a non-equilibrium phase transition between decaying and spreading turbulence. But before continuing with this idea, some experimental challenges of pipe flow experiments are highlighted. The main challenge was posed by the large time scales involved, up to $10^{7}$ advective units at $R e_{c}$. Experimentally this means extremely rare events have to be properly detected, without false positives, and a long-time stability of the setup provided. To determine the mean time of the splitting close to $R e_{c}$, up to 60000 measurements, of which only seven splitted, were performed in a pipe of about $15 \mathrm{~m}$ overall length with a diameter of $4 \mathrm{~mm}$. The triggering of perturbations with a valve to generate puffs, and their detection with pressure sensors (without disturbing the flow in an uncontrolled manner) required intensive careful tests. In addition, the superexponential scaling of the mean time of splitting with the $R e$ implies that any small deviation in $R e$ leads to large uncertainties in splitting time. Such deviations can arise from temperature differences, changing the viscosity of the working fluid (distilled water with copper sulfate), or from the growth of algae, which is substantially more difficult to control. Algae can form biofilms, which act similarly as a polymer, and shift the onset of turbulence to higher $R e$ (Hartung 2012). But especially for measurements close to the critical $R e_{c}$, such a shift can only be detected after several days of measurements. Therefore, a routine was introduced of calibrating the pipe every other day against well-known mean splitting times at higher $R e$, and if a shift occurred the pipe was dismantled and thoroughly cleaned. In addition to developing a sophisticated cleaning method, the distilled water was exchanged after a few days to prevent algae growth instead of several weeks (as it was commonly done before). To have a more than 3000 diameter long pipe flow experiment reliably working for several months, was accomplished for the first time in this thesis. The setup itself, and also the maintenance methods, are now used by other researchers for more detailed investigations around $R e_{c}$.

The onset of sustained turbulence in pipe flow is determined by the competition of two contrary statistical processes, the spreading and the decaying of turbulent patches. Two important conclusions can be drawn from this. The first one is that the temporal aspects (as in Ruelle \& Takens 1971) do not determine the transition. The results of this thesis emphasize the importance of spatial aspects and present a new scenario of the transition to turbulence for shear flows with a linearly stable laminar flow profile. From plane Couette and Taylor-Couette flows it is known that turbulent patches at low $R e$ are transient, but spread at larger Re (Daviaud et al. 1992, Bottin \& Chaté 1998, Coles 1965, Borrero-Echeverry et al. 2010). By comparing the time scales of both these processes the critical $R e_{c}$ for the onset of sustained turbulence could be determined in these systems. The scenario is therefore not only a valid description for pipe flow but most likely for a whole class of shear flows. The second conclusion reaches even further. The transition of such kind of shear flows can be interpreted as non-equilibrium phase transition, which separates the regimes of decaying from spreading turbulence.

The framework of statistical physics opens new ways to study this transition. The 
first question is, if the transition is discontinuous, and therefore of first order, or if it is continuous and therefore of second order. This can be distinguished by looking at the scaling of the mean turbulent fraction with the $R e>R e_{c}$. However, the time scales to reach equilibrium in pipe flow are so long that they can be hardly realized. They are therefore clearly out of the scope of this thesis. Motivated by the results presented in Chapter 2, Barkley (2011) developed a model that reproduces the dynamics of the decaying and spreading of puffs. The transition was found to be of second order with the scaling of the turbulent fraction described by a power law. Moreover, the exponent of the power law is identical within computational accuracy to the one of directed percolation in one dimension. Pomeau (1986) had already pointed out that the transition in pipe flow might be described by directed percolation, but the comparison stayed at a qualitative level. While this idea gave great impulse to the study of shear flows with a linearly stable laminar flow, the plane Couette experiments performed much later yielded a different result. Bottin et al. (1998) suggested the transition to be of first order, which excludes any description with directed percolation models. The recent results of Barkley (2011) have resurrected the idea to describe the onset of turbulence by directed percolation. In plane Couette, or high radius-ratio Taylor-Couette flows, the time scales are several orders of magnitude shorter than in pipe flow (Shi et al. 2013), so that experiments and direct numerical simulations are doable.

In the introduction (Chapter 1) results from literature of the scaling of the turbulent fraction are compared with the one corresponding to directed percolation. It turns out that simulations (Duguet et al. 2010) are not close enough to the critical point to clearly distinguish between a first and a second order phase transition. As discussed in Chapter 4 the accuracy achieved in the experiments was about 3\% in Re (Bottin et al. 1998, Bottin \& Chaté 1998). Moreover, the small system size may have prevented observing a continuous transition. In a qualitative analysis of the influence of system size and accuracy on the phase transition, it has been argued in this thesis that a second order phase transition would always appear discontinuous in an experiment. The system size and accuracy of the setup determine the minimum (non-zero) turbulent fraction that can be measured, or in other words how well the second order phase transition can be approximated. Attempts to build a large system size experiment with a high accuracy failed in the past for both, plane Couette (Prigent et al. 2003) and Taylor-Couette setups (see Chapter 3, Fig. 3.13). The origin of these technical problems lies in the fact that an increasing system size corresponds to a decreasing gap width (see Chapter 1, Fig. 1.17). The smaller the gap compared to the size of the plates or cylinders, the more difficult it is to keep the gap width constant. The accuracy of the gap width on the other hand determines the deviation in $R e$. Such deviations may act as finite amplitude perturbations that trigger the transition to turbulence in the subcritical regime (see Chapter 3, Fig. 3.13). In this thesis a high-precision Taylor-Couette setup (see Chapter 3) has been built with a system size 12 times larger than previous plane Couette experiments (Bottin \& Chaté 1998). The accuracy in $R e$ is better than $1 \%$, which was determined by comparing the onset of instabilities with a linear stability analysis. In contrast to other Taylor-Couette experiments with comparable system sizes (Prigent et al. 2003, Prigent \& Dauchot 2005) in this setup finite amplitude perturbations are indeed avoided in the subcritical regime. This setup satisfies for the first time all conditions to study the phase transition, which was attempted 
by several researchers (assisted by engineers) for more than a decade.

The measurements using this apparatus in Chapter 4 reveal that the phase transition is of second order and not of first order as previously anticipated. Compared to the previously detected minimal turbulent fraction in a plane Couette experiment (of about 35\% Bottin et al. (1998)), the measurements in this thesis reach a five times smaller value (about 7\%). Because of the large system size and high precision, the turbulence was sustained for the whole observation period of $1.4 \cdot 10^{6}$ advective units. The scaling of the turbulent fraction in the vicinity of the critical point $\left(R e \geq R e_{c}+20\right)$ is approximated by a power law with the first critical exponent of directed percolation in two dimensions. Even though this result needs more detailed measurements to be confirmed, the idea that the onset of turbulence in linearly stable shear flows is described by directed percolation is very fascinating.

In future measurements the distributions of the laminar gaps in space and time will be analyzed. According to directed percolation their distribution is expected to follow a power-law scaling with the second and third critical exponent respectively (Hinrichsen $2000 a$ ). However, these scalings can only be observed in the vicinity of the critical $R e_{c}$, far away the scaling is predicted to be exponential (Chaté \& Manneville 1987). This change from a power-law to an exponential scaling is characteristic of directed percolation and was recently numerically observed in a quasi-one dimensional plane Couette system (Shi et al. 2013). Even though this result supports directed percolation as the proper description for the onset of turbulence, no critical exponents were presented in this study that could prove this. Future experiments are already in preparation and will clarify this. The image processing was automatized during this thesis, so that huge amounts of data from the high-speed cameras can be effectively analyzed (see Chapter 3 for details).

Apart from the specific investigation of the phase transition at the onset of turbulence in pipe and Taylor-Couette flow, a major accomplishment of this thesis is the development of the Taylor-Couette device as it is presented in Chapter 3. For the first time this setup has allowed it to accurately study the phase transition as it was already described above. But the main advantage of this setup lies in its exceptional flexibility in Reynolds numbers, geometric dimensions, boundary conditions and temperatures combined with a high-precision and optimal optical access. In the future this device will be used for all kind of investigations concerning transitional and high-Reynolds number flows.

Since this experiment is highly complex, it would be tedious to summarize all the technical difficulties and details in this paragraph (see Chapter 3 for details). Instead I want to point out that it was the hand in hand work with an external engineer (Klaus Rühl, arkkon GmbH, Germany) that led to this design. I used my knowledge of physics and experience in the field of fluid dynamics to derive the objective from the general setup down to details, like the desired precision or specific bearing of an individual piece. From this objectives he developed a design with state-of-the-art products and techniques. Subsequently, I checked the design, and as probably one of the most important steps, I tried to foresee problems of physical or technical nature and suggested specific changes in the design. After many iterations the final design was obtained. The effort was rewarded, on the one hand when it was finally build and only small changes were necessary, and on the other hand, when I obtained results (presented in Chapter 4) that re-opened the path to a new understanding of the onset of turbulence. I am convinced that this device with 
its large flexibility and high-precision, will enable investigations that seemed technically too difficult but are physically highly interesting.

\subsection{Open question: Localization of turbulence}

The results of this thesis are an important step to quantify the transition to turbulence and to place this transition in the group of phase transitions. However, a question of transitional flows that remained completely untouched here is the physical mechanism that leads to the localization of turbulence at low $R e$. The lower energy input at these $\operatorname{Re}$ (Hof et al. 2010) explains the intermittent and therefore localized nature of turbulence compared to higher $R e$, where the flow appears fully turbulent. But what is very puzzling is that the turbulent patches have a similar characteristic size in different systems (Samanta et al. 2011). In pipe flow for example the puffs have a length of about 20-30 diameters (Wygnanski et al. 1975) and in Taylor-Couette and plane Couette flows the turbulent bands have a width of 20-40 gap units (Prigent et al. 2002). The universality of this length in different flows hints at a universal origin of localization. But so far the underlying physical mechanism are not understood.

\section{Localization in pipe flow}

In the recent model of Barkley (2011) the proper behavior of puff decaying and puff splitting is observed, but no physical mechanisms are directly modeled here. Instead, it is based on similar equations as models describing excitable media. In such media a relaxation time leads to a localization of the activity. In pipe flow the relaxation time leads to the localization of turbulence and hence the generation of laminar gaps. Experiments have shown that the physical mechanism behind these gaps is the change of the mean flow profile. Directly downstream of a puff the profile is plug-like. Only at a distance of about 15 pipe diameter the profile has recovered enough towards a Poiseuille profile so that it can sustain a puff again (Samanta et al. 2011, Hof et al. 2010). However, the question why the turbulent puffs are localized with a characteristic length of 20-30 diameters is still open.

\section{Localization in Couette flows}

The combined length of a turbulent stripe and a laminar gap was investigated experimentally in plane and Taylor-Couette flow (Prigent et al. 2002, 2003). This combined length is similar in both systems and decreases for increasing $R e$. The authors interpreted the localization as a result of a supercritical long-wavelength instability. But the origin of this instability and the underlying physical mechanism remained unknown (Prigent et al. 2002). Interestingly the same wavelength/instability was observed for the plane-Couette flow, where the laminar flow is linearly stable in contrast to their measurements in TaylorCouette flow, where the flow was already linearly unstable.

It remains a challenge for the future to gain new insights into this apparently quite universal mechanism. 



\section{Perspective}

In this thesis the oldest question of the onset of turbulence has been answered and the transition of shear flows with a linearly stable laminar flow was placed in the class of non-equilibrium phase transitions. These results have been exclusively obtained by experiment.

However, the growing importance of direct numerical simulations (DNS) for the onset of turbulence should not be underestimated. In this work the main advantage of the experiments compared to simulations are the large system sizes and long observation times. If the numerical and statistical resolution is sufficient and boundary conditions are properly chosen, then there is no doubt that the DNS of the Navier-Stokes equation describes the 'real' flow dynamics. In addition, simulations offer besides the information of the full flow field the opportunity to track and stimulate certain (coherent) structures, which are believed to be the seeds of turbulence (Kawahara et al. 2012). With the quickly growing computing power I therefore assume that in the future main advances in understanding the onset and nature of purely hydrodynamic turbulence in simple geometries will be gained by numerical simulation. The picture is completely different when new physical, biological or chemical effects are added to the flow. New physical effects can e.g. be generated by a magnetic field, particles, non-Newtonian fluids or two-phase flows. These are of paramount importance in applications. Even though DNS are in these cases very challenging, at least the equations describing the behavior are more or less clear. How the onset of turbulence influences biological and chemical processes like reproduction or the developing of cellular structures is far from understood and the equations needed to perform DNS are not available. I believe that in such fields precise, well-controlled experiments in simple geometries are imperative to push the frontiers of knowledge. 



\section{Bibliography}

Allhoff, K. T. \& EckHARdT, B. 2012 Directed percolation model for turbulence transition in shear flows. Fluid Dyn. Res. 44 (3), 031201.

Andereck, C. D., Liu, S. S. \& Swinney, H. L. 1986 Flow regimes in a circular Couette system with independently rotating cylinders. J. Fluid Mech. 164, 155-183.

Avila, K. \& HoF, B. 2013 High-precision Taylor-Couette experiment to study subcritical transitions and the role of boundary conditions and size effects. Rev. Sci. Instrum. 84, 065106.

Avila, K., Moxey, D., de Lozar, A., Avila, M., Barkley, D. \& Hof, B. 2011 The onset of turbulence in pipe flow. Science 333 (6039), 192-196.

AvILA, M. 2012 Stability and angular-momentum transport of fluid flows between corotating cylinders. Phys. Rev. Lett. 108 (12), 124501.

Avila, M., Grimes, M., Lopez, J. M. \& Marques, F. 2008 Global endwall effects on centrifugally stable flows. Phys. Fluids 20, 104104.

Avila, M., Willis, A. P. \& Hof, B. 2010 On the transient nature of localized pipe flow turbulence. J. Fluid Mech. 646, 127.

Barkley, D. 2011 Simplifying the complexity of pipe flow. Phys. Rev. E 84 (1), 016309.

Benjamin, T. B. 1978 Bifurcation phenomena in steady flows of a viscous fluid. I. Theory. II. Experiments. Proc. Roy. Soc. Lond. A 359, 1-43.

Blackburn, H. M. \& Sherwin, S. J. 2004 Formulation of a Galerkin spectral elementFourier method for three-dimensional incompressible flows in cylindrical geometries. J. Comput. Phys. 197 (2), 759-778.

Blasıus, H. 1911 Das Ähnlichkeitsgesetz bei Reibungsvorgängen. Phys. Z. 12, 11751177.

Bodenschatz, E., de Bruyn, J.R., Ahlers, G. \& Cannell, D. S. 1991 Transitions between patterns in thermal convection. Phys. Rev. Lett. 67 (22), 3078.

Borrero-Echeverry, D., Schatz, M. F. \& Tagg, R. 2010 Transient turbulence in TaylorCouette flow. Phys. Rev. E 81 (2), 025301. 
Bottin, S. \& Chaté, H. 1998 Statistical analysis of the transition to turbulence in plane Couette flow. Europ. Phys. J. B 6 (1), 143-155.

Bottin, S., Daviaud, F., Manneville, P. \& Dauchot, O. 1998 Discontinuous transition to spatiotemporal intermittency in plane Couette flow. Europhys. Lett. 43 (2), 171.

BRADShaw, P. 1969 The analogy between streamline curvature and buoyancy in turbulent shear flow. J. Fluid Mech. 36 (01), 177-191.

BramfitT, B. L. 2001 Metallographer's guide: practices and procedures for irons and steels. ASM International.

Brauckmann, H. J. \& Eckhardt, B. 2013 Intermittent boundary layers and torque maxima in Taylor-Couette flow. Phys. Rev. E 87 (3), 033004.

Broadbent, R. \& Hammersley, J. M. 1957 Percolation processes. The Annals of Mathematical Statistics 28 (3), 629-641.

Brosa, U. 1989 Turbulence without strange attractor. J. Stat. Phys. 55 (5-6), 1303-1312.

Brown, L. D., CaI, T. T. \& DasGupta, A. 2001 Interval estimation for a binomial proportion. Stat. Science 16 (2), 101-133.

Burin, M. J. \& Czarnocki, C. J. 2012 Subcritical transition and spiral turbulence in circular Couette flow. J. Fluid Mech. 709, 106-122.

Carey, C. S., Schlender, A. B. \& Andereck, C. D. 2007 Localized intermittent shortwavelength bursts in the high-radius ratio limit of the Taylor-Couette system. Phys. Rev. E 75 (1), 016303.

Carlson, J. A., Jaffee, A. \& Wiles, A. J. 2006 The millennium prize problems. American Mathematical Society Bookstore.

Chandrasekhar, S. 1961 Hydrodynamic and hydromagnetic stability, Intern. Series Monographs Phys., vol. 1. Oxford: Clarendon.

Chaté, H. \& Manneville, P. 1987 Transition to turbulence via spatio-temporal intermittency. Phys. Rev. Lett. 58 (2), 112-115.

Chaté, H. \& Manneville, P. 1988 Spatio-temporal intermittency in coupled map lattices. Physica D 32 (3), 409-422.

Ciliberto, S. \& Bigazzi, P. 1988 Spatiotemporal intermittency in Rayleigh-Bénard convection. Phys. Rev. Lett. 60, 286-289.

Clever, R. M. \& Busse, F. H. 1974 Transition to time-dependent convection. J. Fluid Mech. 65 (4), 625-645.

Clever, R. M. \& Busse, F. H. 1997 Tertiary and quaternary solutions for plane Couette flow. J. Fluid Mech. 344, 137-153. 
Coles, D. 1962 Interfaces and intermittency in turbulent shear flow. Mécanique de la Turbulence 108, 229.

Coles, D. 1965 Transition in circular Couette flow. J. Fluid Mech. 21, 385-425.

Colovas, P. W. \& Andereck, C. D. 1997 Turbulent bursting and spatiotemporal intermittencyin the counterrotating Taylor-Couette system. Phys. Rev. E 55, 2736-2741.

Couette, M. 1890 Etudes sur le frottement des liquides. Ann. Chim. Phys. 21 (433).

Cross, M. C. \& Hohenberg, P. C. 1993 Pattern formation outside of equilibrium. Rev. Mod. Phys. 65 (3), 851.

DarbyshiRe, A. G. \& Mullin, T. 1995 Transition to turbulence in constant-mass-flux pipe flow. J. Fluid Mech. 289, 83-114.

DARcy, H. 1857 Recherches expérimentales relatives au mouvement de l'eau dans les tuyaux, , vol. 1. Mallet-Bachelier.

Davey, A., Prima, R. C. Di \& Stuart, J. T. 1968 On the instability of Taylor vortices. J. Fluid Mech. 31 (01), 17-52.

Daviaud, F., Bonetti, M. \& Dubois, M. 1990 Transition to turbulence via spatiotemporal intermittency in one-dimensional Rayleigh-Bénard convection. Physi. Rev. A 42 (6), 3388.

Daviaud, F., Hegseth, J. \& Bergé, P. 1992 Subcritical transition to turbulence in plane Couette flow. Phys. Rev. Lett. 69 (17), 2511.

Degen, M., Mutabazi, I. \& Andereck, C. D. 1996 Transition to weak turbulence via spatiotemporal intermittency in the Taylor-Dean system. Phys. Rev. E 53 (4), 3495.

Di Prima, R. C. \& Swinney, H. L. 1985 Instabilities and transition in flow between concentric rotating cylinders. In Hydrodynamic instabilities and the transition to turbulence, pp. 139-180. Springer.

Dominguez-Lerma, M. A., Cannell, D. S. \& Ahlers, G. 1986 Eckhaus boundary and wave-number selection in rotating Couette-Taylor flow. Phys. Rev. A 34 (6), 4956.

Donnelly, R. J. 1991 Taylor-Couette flow: The early days. Phys. Today 44 (11), 32-39.

Drazin, P. G. \& ReID, W. H. 2004 Hydrodynamic stability. Cambridge University Press.

Dubrulle, B. \& Hersant, F. 2002 Momentum transport and torque scaling in TaylorCouette flow from an analogy with turbulent convection. Europ. Phys. J. B 26 (3), 379-386.

Duguet, Y., Schlatter, P. \& Henningson, D. S. 2010 Formation of turbulent patterns near the onset of transition in plane Couette flow. J. Fluid Mech. 650, 119.

Durst, F. \& ÜnsAL, B. 2006 Forced laminar-to-turbulent transition of pipe flows. J. Fluid Mech. 560, 449-464. 
EckHARDT, B. 2008 Turbulence transition in pipe flow: Some open questions. Nonlinearity 21 (1), T1.

EcKHARDT, B. 2009 Introduction. Turbulence transition in pipe flow 125th anniversary of the publication of Reynolds' paper. Phil. Trans. Roy. Soc. Lond. A 367 (1888), 449-455.

Eckhardt, B., Schneider, T. M., Hof, B. \& Westerweel, J. 2007 Turbulence transition in pipe flow. Ann. Rev. Fluid Mech. 39, 447-468.

Euler, LeONHARD 1752 Découverte d'un nouveau principe de mécanique.

Faisst, H. \& Eckhardt, B. 2000 Transition from the Couette-Taylor system to the plane Couette system. Phys. Rev. E 61 (6), 7227.

Faisst, H. \& Eckhardt, B. 2004 Sensitive dependence on initial conditions in transition to turbulence in pipe flow. J. Fluid Mech. 504, 343-352.

van Gils, Dennis P. M., Bruggert, G. W., Lathrop, D. P., Sun, C. \& Lohse, D. $2011 a$ The Twente turbulent Taylor-Couette (T 3 C) facility: Strongly turbulent (multiphase) flow between two independently rotating cylinders. Rev. Sci. Instr. 82 (2), 025105-025105.

van Gils, D. P. M., Huisman, S. G., Bruggert, G. W., Sun, C. \& Lohse, D. $2011 b$ Torque scaling in turbulent Taylor-Couette flow with co-and counterrotating cylinders. Phys. Rev. Lett. 106 (2), 24502.

Gollub, J. P. \& Swinney, H. L. 1975 Onset of turbulence in a rotating fluid. Phys. Rev. Lett. 35 (14), 927.

Grassberger, P. 1982 On phase transitions in Schlögl's second model. Z. f. Phys. B 47 (4), $365-374$.

Hagen, G. 1839 Über die Bewegung des Wassers in engen zylindrischen Röhren. Ann. Phys. Chem. 46, 423-442.

Hagen, G. 1854 Über den Einfluss der Temperatur auf die Bewegung des Wassers in Rohren. Abh. Akad. Wiss., Berlin p. 17.

Hammersley, J. M. 1957 Percolation processes. Math. Proc. Cambridge Phil. Soc. 53 (3), $642-645$.

Harris, T. E. 1974 Contact interactions on a lattice. Ann. Prob. 2 (6), 969-988.

Hartung, J. 2012 Biofilme in Scherströmung. Master's thesis, Georg August University, Göttingen, Germany.

Hegseth, J. J., Andereck, C. D., Hayot, F. \& Pomeau, Y. 1989 Spiral turbulence and phase dynamics. Phys. Rev. Lett. 62 (3), 257-260.

Heise, M., Hochstrate, K., Abshagen, J. \& Pfister, G. 2009 Spirals vortices in TaylorCouette flow with rotating endwalls. Phys. Rev. E 80 (4), 045301. 
Hinrichsen, H. $2000 a$ Non-equilibrium critical phenomena and phase transitions into absorbing states. Advances in Physics 49 (7), 815-958.

HinRichsen, H. $2000 b$ On possible experimental realizations of directed percolation. Brazilian Journal of Physics 30 (1), 69-82.

Hof, B., De Lozar, A., Kuik, D. J. \& Westerweel, J. 2008 Repeller or attractor? selecting the dynamical model for the onset of turbulence in pipe flow. Phys. Rev. Lett. 101 (21), 214501.

Hof, B., van Doorne, C. W. H., Westerweel, J. \& Nieuwstadt, F. T. M. 2005 Turbulence regeneration in pipe flow at moderate Reynolds numbers. Phys. Rev. Lett. 95 (21), 214502.

Hof, B., Juel, A. \& Mullin, T. 2003 Scaling of the turbulence transition threshold in a pipe. Phys. Rev. Lett. 91 (24), 244502.

Hof, B., de Lozar, A., Avila, M., Tu, X. \& Schneider, T. M. 2010 Eliminating turbulence in spatially intermittent flows. Science 327 (5972), 1491-1494.

Hof, B., Westerweel, J., Schneider, T. M. \& Eckhardt, B. 2006 Finite lifetime of turbulence in shear flows. Nature 443 (7107), 59-62.

JANSSEN, H. K. 1981 On the nonequilibrium phase transition in reaction-diffusion systems with an absorbing stationary state. Zeitschr. Phys. B 42 (2), 151-154.

Ji, H., Burin, M., Schartman, E. \& Goodman, J. 2006 Hydrodynamic turbulence cannot transport angular momentum effectively in astrophysical disks. Nature 444 (05323), 343.

Joseph, D. D. 1976 Global stability of Couette flow between rotating cylinders. In Stability of Fluid Motions I, pp. 130-157. Springer.

Kaneko, K. 1985 Spatiotemporal intermittency in coupled map lattices. Prog. Theor. Phys. 74 (5), 1033-1044.

von Kármán, Theodor 1954 Aerodynamics, Selected Topics in the Light of Their Historical Development.. NY, Cornell University Press.

Kawahara, G., Uhlmann, M. \& van Veen, L. 2012 The significance of simple invariant solutions in turbulent flows. Ann. Rev. Fluid Mech. 44, 203-225.

Klahr, H. H. \& Bodenheimer, P. 2003 Turbulence in accretion disks: Vorticity generation and angular momentum transport via the global baroclinic instability. Astrophys. $J$. $582(2), 869$.

Kuik, D. J., Poelma, C. \& Westerweel, J. 2010 Quantitative measurement of the lifetime of localized turbulence in pipe flow. J. Fluid Mech. 645 (1), 529-539.

Landau, L. D. 1944 On the problem of turbulence. CR Acad. Sci. URSS 44 (31), 1-314. 
Landau, L. D. \& Lifshitz, E. 1959 Fluid Mechanics, , vol. 225. Pergamon Press Oxford, England.

Lathrop, D. P., Fineberg, J. \& Swinney, H. L. 1992 Turbulent flow between concentric rotating cylinders at large Reynolds number. Phys. Rev. Lett. 68 (10), 1515-1518.

Lawless, J. F. 2003 Statistical Models and Methods for Lifetime Data, 2nd edn. New Jersey: Wiley.

LoRenz, E. N. 1963 Deterministic nonperiodic flow. Journal of the Atmospheric Sciences 20 (2), 130-141.

DE Lozar, A. \& Hof, B. 2009 An experimental study of the decay of turbulent puffs in pipe flow. Phil. Trans. Roy. Soc. Lond. A 367 (1888), 589-599.

Mallock, A. 1888 Determination of the viscosity of water. Proc. Roy. Soc. Lond. A 45 (273-279), 126-132.

Mallock, A. 1896 Experiments on fluid viscosity. Phil. Trans. Roy. Soc. Lond. A 187, 41-56.

Manneville, P. 2009 Spatiotemporal perspective on the decay of turbulence in wallbounded flows. Phys. Rev. E 79 (2), 25301.

Merbold, S., Brauckmann, H. J. \& Egbers, C. 2013 Torque measurements and numerical determination in differentially rotating wide gap Taylor-Couette flow. Phys. Rev. E 87, 023014.

Meseguer, A. \& Trefethen, L. N. 2003 Linearized pipe flow to Reynolds number 10,000,000. J. Comput. Phys. 186 (1), 178-197.

Moxey, D. \& Barkley, D. 2010 Distinct large-scale turbulent-laminar states in transitional pipe flow. Proc. Natl. Acad. Sci. USA 107 (18), 8091.

Mullin, T. 2011 Experimental studies of transition to turbulence in a pipe. Ann. Rev. Fluid Mech. 43, 1-24.

NagATa, M. 1990 Three-dimensional finite-amplitude solutions in plane Couette flow: Bifurcation from infinity. J. Fluid Mech. 217, 519-527.

Navier, C. L. 1823 Mémoire sur les lois du mouvement des fluides. Mémoires de l'Académie Royale des Sciences de l'Institut de France 6, 389-440.

Nishi, M., ÜNSAL, B., Durst, F. \& Biswas, G. 2008 Laminar-to-turbulent transition of pipe flows through puffs and slugs. J. Fluid Mech. 614, 425-446.

Pacheco, J. R., Lopez, J. M. \& Marques, F. 2011 Pinning of rotating waves to defects in finite Taylor-Couette flow. J. Fluid Mech. 666, 254.

Paoletti, M. S., van Gils, D. P. M., Dubrulle, B., Sun, C., Lohse, D. \& Lathrop, D. P. 2012 Angular momentum transport and turbulence in laboratory models of Keplerian flows. Astr. and Astrophys. 547, A64. 
Paoletti, M. S. \& Lathrop, D. P. 2011 Measurement of angular momentum transport in turbulent flow between independently rotating cylinders. Phys. Rev. Lett. 106, 024501.

Peixinho, J. \& Muluin, T. 2006 Decay of turbulence in pipe flow. Phys. Rev. Lett. 96 (9), 094501.

Pfenninger, W. 1961 Boundary layer suction experiments with laminar flow at high Reynolds numbers in the inlet length of a tube by various suction methods. Boundary layer and flow control 2, 961-980.

Pfister, G. \& Rehberg, I. 1981 Space-dependent order parameter in circular Couette flow transitions. Phys. Lett. A 83 (1), 19-22.

Poiseuille, J. L. M. 1840 Recherches expérimentales sur le mouvement des liquides dans les tubes de très petits diametrès: I. influence de la pression sur la quantité de liquide qui traverse les tubes de très petits diametrès. C. R. Acad. Sci. 11, 961-967.

Pomeau, Y. 1986 Front motion, metastability and subcritical bifurcations in hydrodynamics. Physica D 23 (1-3), 3-11.

Prigent, A. \& Dauchot, O. 2000a Barber pole turbulence. arXiv: cond-mat/0009241 v1 15.

Prigent, A. \& Dauchot, O. $2000 b$ Visualization of a Taylor-Couette flow avoiding parasitic reflections. Phys. Fluids 12 (10), 2688-2690.

Prigent, A. \& Dauchot, O. 2005 Transition to versus from turbulence in subcritical couette flows. In IUTAM Symposium on Laminar-Turbulent Transition and Finite Amplitude Solutions, pp. 195-219. Springer.

Prigent, A., Grégoire, G., Chaté, H. \& Dauchot, O. 2003 Long-wavelength modulation of turbulent shear flows. Physica D 174 (1), 100-113.

Prigent, A., Grégoire, G., Chaté, H., Dauchot, O. \& van Saarloos, W. 2002 Largescale finite-wavelength modulation within turbulent shear flows. Phys. Rev. Lett. 89 (1), 14501.

Ravelet, F., Delfos, R. \& Westerweel, J. 2010 Influence of global rotation and Reynolds number on the large-scale features of a turbulent Taylor-Couette flow. Phys. Fluids 22, 055103.

RAYLEIGH, LORD 1916 LIX. On convection currents in a horizontal layer of fluid, when the higher temperature is on the under side. Phil. Magazine Series 632 (192), 529-546.

Rayleigh, Lord 1917 On the dynamics of revolving fluids. Proc. Roy. Soc. Lond. A 93 (648), 148-154.

ReYnolds, O. 1883 An experimental investigation of the circumstances which determine whether the motion of water shall be direct or sinuous, and of the law of resistance in parallel channels. Proc. Roy. Soc. Lond. 35 (224-226), 84-99. 
ReYnolds, O. 1895 On the dynamical theory of incompressible viscous fluids and the determination of the criterion. Phil. Trans. Roy. Soc. Lond. A 186, 123-164.

RITwIK 2012 Measuring the viscous flow behaviour of molten metals under shear. Brunel Centre for Advanced Solidification Technology .

Rolf, J., Bohr, T. \& Jensen, M. H. 1998 Directed percolation universality in asynchronous evolution of spatiotemporal intermittency. Phys. Rev. E 57 (3), R2503-R2506.

Romanov, V.A. 1973 Stability of plane-parallel Couette flow. Functional Analysis and its Applications 7 (2), 137-146.

Rotт, N. 1990 Note on the history of the Reynolds number. Ann. Rev. Fluid Mech. 22 (1), $1-12$.

RotтA, J. 1956 Experimenteller Beitrag zur Entstehung turbulenter Strömung im Rohr. Arch. Appl. Mech. 24 (4), 258-281.

Ruelle, D. \& Takens, F. 1971 On the nature of turbulence. Commun. Math. Phys. 20 (3), 167-192.

Salwen, H., Cotton, F. W. \& Grosch, C. E. 1980 Linear stability of Poiseuille flow in a circular pipe. J. Fluid Mech. 98 (2), 273-284.

Samanta, D., de Lozar, A. \& Hof, B. 2011 Experimental investigation of laminar turbulent intermittency in pipe flow. J. Fluid Mech. 681, 193-204.

Schartman, E., Ji, H. \& Burin, M. J. 2009 Development of a Couette-Taylor flow device with active minimization of secondary circulation. Rev. Sci. Instrum. 80, 024501.

Schultz-Grunow, F. 1959 Zur Stabilität der Couette-Strömung. Zeitschrift für Angewandte Mathematik und Mechanik 39 (3-4), 101-110.

ShI, L., Avila, M. \& Hof, B. 2013 Scale invariance at the onset of turbulence in Couette flow. Phys. Rev. Lett. 110 (20), 204502.

SoMmERFELD, A. 1908 Ein Beitrag zur hydrodynamischen Erklärung der turbulenten Flüssigkeitsbewegungen. In Int. Congr. Math.,4th, Rome, , vol. 3, pp. 116-124.

Stokes, G. G. 1845 On the theories of internal friction of fluid in motion, and of the equilibrium and motion of elastic solids. Trans. Cambridge Philos. Soc 8, 287.

Swinney, H. L. \& Gollub, J. P. 1978 The transition to turbulence. Phys. Today 31, 41.

Takeuchi, K. A., Kuroda, M., Chaté, H. \& Sano, M. 2007 Directed percolation criticality in turbulent liquid crystals. Phys. Rev. Lett. 99 (23), 234503.

TAYLOR, G. I. 1923 Stability of a viscous liquid contained between two rotating cylinders. Phil. Trans. R. Soc. A. 223, 289-343.

TAYLOR, G. I. 1936 Fluid friction between rotating cylinders. I. Torque measurements. Proc. Roy. Soc. Lond. A 157 (892), 546-564. 
TiETJEns, O. 1970 Strömungslehre, Zweiter Band: Bewegung der Flüssigkeiten und Gase. Springer-Verlag.

Tillmark, N. \& Alfredsson, P. 1992 Experiments on transition in plane Couette flow. J. Fluid Mech. 235, 89-102.

Voigt, C. A. \& ZIFF, R. M. 1997 Epidemic analysis of the second-order transition in the Ziff-Gulari-Barshad surface-reaction model. Phys. Rev. E 56 (6), R6241.

Willis, A. P. \& Kerswell, R. R. 2007 Critical behavior in the relaminarization of localized turbulence in pipe flow. Phys. Rev. Lett. 98 (1), 014501.

Willis, A. P. \& Kerswell, R. R. 2009 Turbulent dynamics of pipe flow captured in a reduced model: Puff relaminarization and localized 'edge' states. J. Fluid Mech. 619, 213-233.

Wygnanski, I. J. \& Champagne, F. H. 1973 On transition in a pipe. Part 1. The origin of puffs and slugs and the flow in a turbulent slug. J. Fluid Mech. 59 (02), 281-335.

Wygnanski, I. J., Sokolov, M. \& Friedman, D. 1975 On transition in a pipe. Part 2. The equilibrium puff. J. Fluid Mech. 69 (02), 283-304. 



\section{Acknowledgments}

My first acknowledgement is directed to my supervisor Prof. Hof. Working together with him is the best experience I have so far in my scientific life. His way of identifying key questions in our research field, combined with his enthusiasm to solve them sparing no effort, has shaped my own attitude towards science. I appreciate very much that, on the one hand he was always present to discuss anything, but, on the other hand, gave me almost complete freedom on how I built, performed and analyzed experiments or presented the results.

What I liked most about Prof. Bodenschatz besides scientific and technical discussions is his way of opening my mind towards a career I have never thought could be possible for me. Conversations with him have been very valuable for me on my way to become an independent researcher.

I also thank him, together with Prof. Herminghaus, for providing an inspiring and in all aspects supportive environment at the Max Planck Institute.

I thank Prof. Tilgner for valuable comments and questions in the thesis meetings. Unfortunately the time was too short to work together on astrophysical problems, which I'm sure I would have enjoyed.

I thank Dr. Alberto de Lozar for discussions and introducing me into pipe flow experiments. Sharing some Lab View programs with me and revealing many small technical tricks saved me a lot of time and ensured a quick start in my PhD-project.

I thank Prof. Eckhardt for many inspiring scientific discussions and his deep interest in supporting young researchers in all ways. Even though he was officially not a cosupervisor, it always felt as if he was one.

During my PhD I met many researchers at conferences, meetings and summer schools. Discussions with them gave often new impulses to my work and their enthusiasm was very motivating for me. As an example I cite an e-mail of Prof. Kaneko, the inventor of coupled map lattices. 'Your talk was very much exciting to me, and I found some of my dreams about 25 years ago have come true, and experimental results are furthermore stimulating beyond my expectation.' 
I thank Dave Moxey and Prof. Barkley for the cooperation in the pipe project. It was a great experience to share the excitement and effort to work together on a high-impact project.

I acknowledge Klaus Rühl for the fruitful cooperation in designing and building the Taylor-Couette experiment. His engineering knowledge was indispensable to push the technical limitations, which was necessary to combine Björns ideas and wishes with mines. Many thanks to the team of the machine shop for doing a great job and especially to the foreman Joachim Hesse for teaching me basic skills in material machining. I also thank Jens Pick and Torsten Harenkamp for help if I needed a quick technical solution. I thank Barbara Kutz for her quick and efficient support in officialism and formalities. It was great to have her as a secretary. I acknowledge the GGNB team for making an effort to support 'their' students in all aspects. I always felt somehow 'home' in this program.

I always enjoyed the activities and discussions inside and outside of work with Markus Holzner, Tobias Lapp, Devranian Samanta, Liang Shi, and all the other members of the group that came later.

I thank Fabio di Lorenzo, Quentin Brosseau, Daniel Feldmann, Daniel Herde, Susanne Horn and Matthew Salewski for organizing the '5th European Postgraduate Fluid Dynamics Conference' here in Göttingen. It was a fantastic team and an outstanding experience to manage such a large event together.

\section{Last but not least}

I'm grateful to my husband Marc for being exactly the way he is: for his love and criticism (including scientific discussions), for making me laugh almost every day and giving me comfort in difficult times.

I dedicate this work to my mother, who died during my Diplom studies. Together with my father she fully supported me during my study, even though she could never believe I would become a 'real physicist'. She would have been proud to see that finally I have become one. 


\section{A Von Wirbelballen zur Turbulenz}

Citation and credit: Erstveröffentlichung dieses Textes in Spektrum der Wissenschaft 3/2012, S.16-18. Reprinted with permission by Spektrum der Wissenschaft Verlagsgesellschaft mbH, Heidelberg, 2013.

Title: 'Von Wirbelballen zur Turbulenz'

Authors: K. Avila and B. Hof

Contribution: I wrote this article together with B. Hof about the results presented in Chapter 2, which are published in Science.

Endlich gelang es Forschern, eine mehr als 100 Jahre alte Frage der Strömungsdynamik zu beantworten: Wann gewinnen in Rohrströmungen Turbulenzen die Oberhand?

Manch scheinbar simple Frage hat in der Wissenschaft schon Generationen von Forschern beschäftigt, die vergeblich nach der Antwort gesucht, dabei aber fundamentale Erkenntnisse gewonnen haben. Ein gutes Beispiel dafür bietet die Fluiddynamik, die Wissenschaft von der Bewegung von Flüssigkeiten und Gasen. Schon vor über 120 Jahren wollte Osborne Reynolds (1842 - 1912) an der University of Manchester herausfinden, wann eine Flüssigkeit nicht mehr glatt durch ein Rohr strömt, sondern Wirbel bildet, die den Strömungswiderstand drastisch ansteigen lassen.

Mit seinen Untersuchungen hat der britische Ingenieur bahnbrechende Fortschritte in der Fluiddynamik erzielt, ohne jedoch seine Ausgangsfrage definitiv beantworten zu können. Das Rätsel blieb bis vor Kurzem ungelöst. Eine vermeintliche Lösung schaffte es zwar in etliche wissenschaftliche Veröffentlichungen, Lehrbücher und sogar in Wikipedia. Doch beruht sie auf einer inkorrekt zitierten Zahl.

In der Fluiddynamik unterscheidet man zwischen laminaren und turbulenten Strömungen. In ersteren bewegt sich die Flüssigkeit glatt in Schichten, die sich nicht vermischen. Meist ist die Strömungsgeschwindigkeit zeitlich konstant. Man denke etwa an Honig, der von einem Löffel fließt. Turbulente Strömungen sind dagegen stark fluktuierend und verwirbelt, was unter anderem zu deutlich höheren Reibungsverlusten führt.

Um den Übergang zwischen den beiden Strömungsarten zu erforschen, wählte Reynolds einen Versuchsaufbau mit möglichst einfacher Geometrie und großer technischer Bedeutung: ein gerades, glattes Glasrohr mit rundem Querschnitt, durch das er unterschiedlich warmes Wasser strömen ließ. Durch Einspritzen von Tinte konnte der britische Forscher die Bewegung sichtbar machen und so eindeutig zwischen laminaren und turbu- 


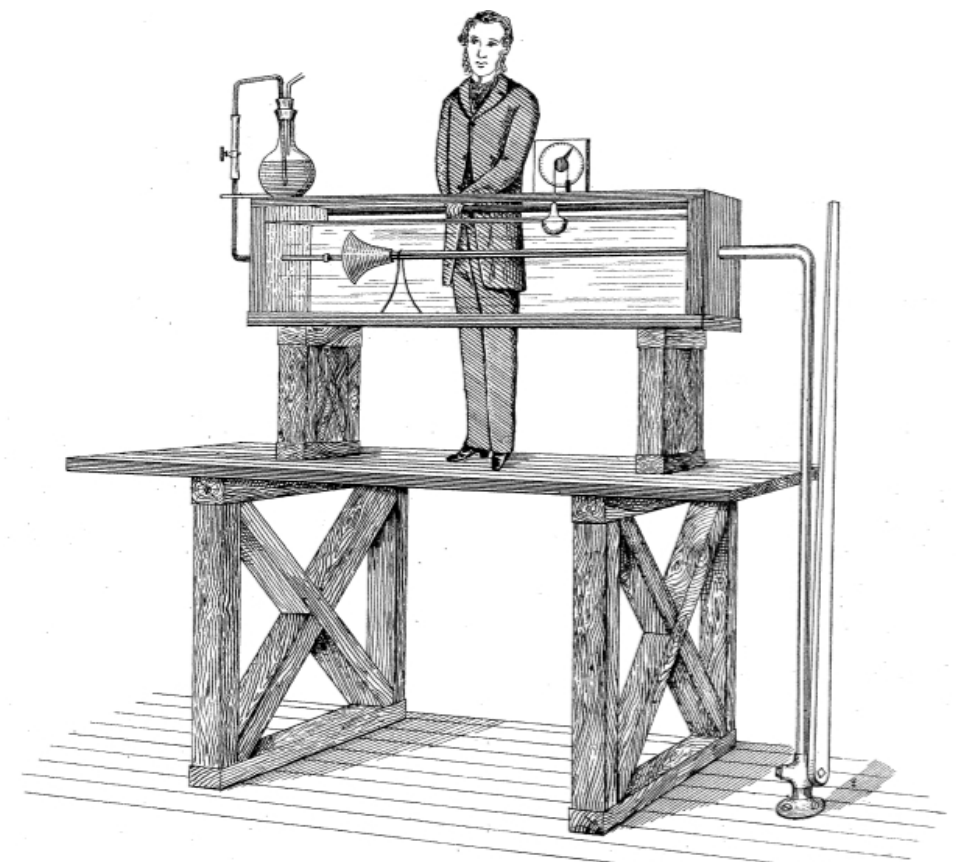

Figure A.1: Auf der historischen Zeichnung ist Osborne Reynolds bei seinen bahnbrechenden Versuchen zur Strömung von Flüssigkeiten in Rohren zu sehen.

lenten Regionen unterscheiden. Wie er feststellte, spielte die Strömungsgeschwindigkeit eine entscheidende Rolle. Erst wenn sie relativ hoch war, bildeten sich Wirbel. Außerdem bemerkte Reynolds, dass ein großer Rohrdurchmesser und eine geringe Viskosität - also Zähigkeit - der Flüssigkeit Turbulenzen begünstigten. Das Verhalten der Strömung hing demnach vom Verhältnis der drei Größen zueinander ab.

\section{Bedeutendes Lebenswerk, doch ohne krönenden Abschluss}

Dies führte Reynolds zu dem wichtigsten Parameter der Fluiddynamik: der später nach ihm benannten Reynoldszahl. Die dimensionslose Größe ist gleich dem Produkt aus Strömungsgeschwindigkeit und Rohrdurchmesser, geteilt durch die Viskosität. Auf ihr beruht das reynoldssche Ähnlichkeitsgesetz, wonach es möglich ist, von Beobachtungen an kleinen Modellen - etwa im Windkanal - auf das Verhalten des Originalsystems zu schließen. Dieses Gesetz bildet eine wesentliche Grundlage der experimentellen Strömungsforschung.

Die Messungen von Reynolds zeigten allerdings auch, dass äußere Störungen - etwa Wellen im Tank, der das Rohr speist - großen Einfluss darauf haben, wann die Turbulenz einsetzt. So konnten Strömungen bei gleicher Reynoldszahl mal verwirbelt und mal laminar sein. Die Frage, ab welcher Geschwindigkeit - bei gegebener Rohrdicke und Viskosität - eine Strömung turbulent wird, hängt somit stark vom Versuchsaufbau ab und lässt sich daher nicht eindeutig beantworten. Reynolds postulierte allerdings, dass es einen kritischen Wert für die Reynoldszahl $\left(R e_{c}\right)$ geben müsse, oberhalb dessen sich vorhandene Wirbel ausbreiten und zu dauerhafter Turbulenz führen, während sie unterhalb dieses Werts zerfallen, so dass die Strömung schließlich in den laminaren Zustand 


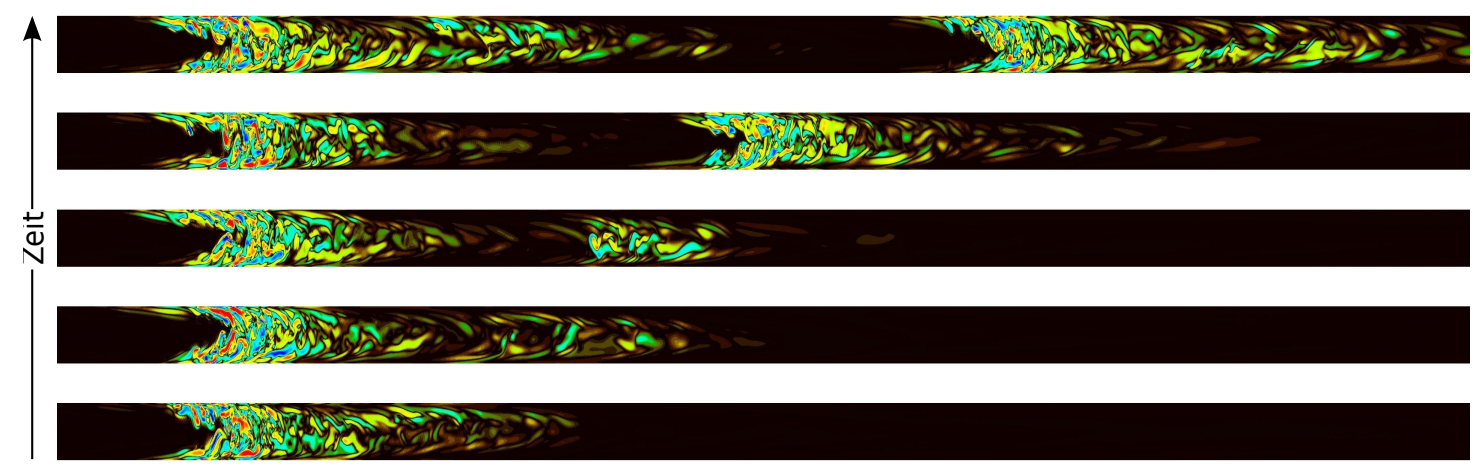

Figure A.2: Die Schnappschüsse einer Computersimulation zeigen von unten nach oben, wie aus einem wachsenden Wirbelballen - einem 'puff' - ein zweiter hervorgeht. Die Flüssigkeit strömt von links nach rechts. Das Bild wurde von M. Avila zur Verfügung gestellt.

zurückkehrt.

Trotz langjähriger Forschung gelang es dem britischen Forscher aber nicht, diese Zahl zu bestimmen. Seine letzten Schätzungen lagen zwischen 1900 und 2000. In diesem Bereich wird die Strömung nie vollständig turbulent. Vielmehr bleiben zwischen den lokalisierten Wirbelballen, so genannten 'puffs', die eine typische Länge von 20 bis 30 Rohrdurchmessern haben, stets laminare Bereiche bestehen (Bild oben).

Unter der Vielzahl von Versuchen, diesen kritischen Punkt zu bestimmen, fanden Experimente besondere Beachtung, die Julis C. Rotta (1912 - 2005) 70 Jahre später an der Aeronautischen Versuchsanstalt in Göttingen durchführte. Sie galten dem Bereich oberhalb von $R e_{c}$, in dem sich die turbulenten Regionen auf Kosten der laminaren ausbreiten. Rotta verfolgte, wie diese Ausbreitungsgeschwindigkeit abnahm, während er die Reynoldszahl allmählich senkte; bei $R e_{c}$ sollte sie gleich null sein. Doch er kam nie so weit, weil sein Rohr zu kurz war und er nicht genügend Experimente durchführen konnte. Seine letzte Messung bei Re $=2300$ ergab noch eine Ausbreitungsgeschwindigkeit von zwei Prozent der mittleren Strömung. Deshalb konnte Rotta das gesuchte $R_{c}$ nur extrapolieren. In seiner Veröffentlichung schrieb er: 'Der genaue Wert dieser kritischen Reynoldszahl kann aus vorliegenden Versuchen nicht bestimmt werden; er mag bei $R e_{c}=2000$ liegen.' Dennoch zitierten viele Wissenschaftler das Ergebnis seiner letzten Messung fälschlicherweise als angebliche Schwelle für den Einsatz der Turbulenz bei der Rohrströmung. Die Zahl 2300 fand so auch Eingang in Lehrbücher und Lexika - darunter Wikipedia. Da das Problem gelöst schien, beschäftigte sich in der Folge kaum noch jemand damit. Das änderte sich erst, als 2004 theoretische Physiker um Bruno Eckhardt von der Universität Marburg neue Erkenntnisse über das Verhalten der Strömung unterhalb von $R e_{c}$ vorlegten. Demnach handelt es sich beim Zerfall der Turbulenz im Rohr um einen statistischen Vorgang mit einer exponentiellen Wahrscheinlichkeitsverteilung. Er findet also vollkommen zufällig statt. Genau wie beim Zerfall radioaktiver Substanzen lässt sich aber eine mittlere Zeit angeben, nach der die Turbulenz zur Hälfte zerfallen ist.

\section{Gleichgewicht zwischen Ausbreitung und Zerfall}

Die exakte Bestimmung dieser Halbwertszeit für jede Reynoldszahl erfordert allerdings 


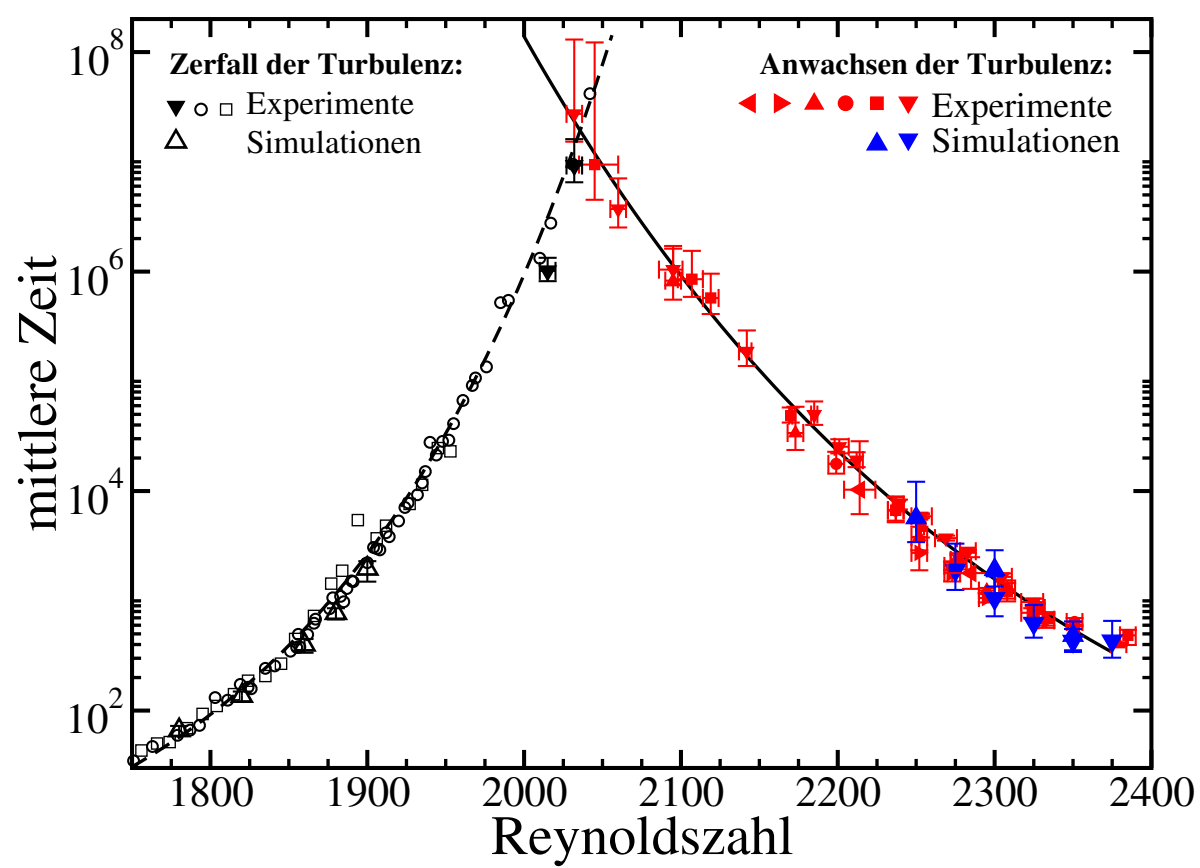

Figure A.3: Ausbreitung und Zerfall von Turbulenzen sind Zufallsprozesse, deren Wahrscheinlichkeit von der Reynoldszahl abhängt. Wo sie sich die beiden Vorgänge die Waage halten - also am Schnittpunkt der beiden Wahrscheinlichkeitskurven -, liegt die kritische Reynoldszahl $R e_{c}$, die den Übergang zwischen laminarer und turbulenter Strömung kennzeichnet.

Tausende bis Hunderttausende von Messungen. Viele Forschergruppen auf der ganzen Welt unterzogen sich dieser Mühe - mit teils widersprüchlichen Ergebnissen. Die Hauptschwierigkeit bestand darin, über Zigtausende von Messungen hinweg alle Versuchsbedingungen exakt konstant zu halten. Ziel der Experimente war es, festzustellen, bei welcher Reynoldszahl die mittlere Zerfallszeit unendlich groß wird. Dies sollte das gesuchte $R e_{c}$ sein. Doch zur großen Enttäuschung der Forscher stiegen die Zerfallszeiten mit zunehmender Reynoldszahl zwar sehr schnell an (linker Ast in Grafik A.3), erreichten aber nie unendlich. Somit ließ sich $R e_{c}$ auf diese Weise nicht bestimmen. Den Durchbruch brachte schließlich die Erkenntnis, dass sich Turbulenz auch schon bei unerwartet niedrigen Reynoldszahlen ausbreiten kann, indem sich Wirbel von einem existierenden 'puff' ablösen und einen neuen bilden (Bild A.2). Wie die Beobachtungen unserer Gruppe an einem 15 Meter langen Glasrohr mit vier Millimeter Durchmesser ergaben, geschieht das jedoch extrem selten. Deshalb bleibt ein turbulenter Bereich oft tagelang unverändert, bevor er plötzlich einen zweiten erzeugt. Wie eine statistische Untersuchung ergab, handelt es sich hierbei ebenfalls um einen Zufallsprozess mit exponentieller Wahrscheinlichkeitsverteilung (Journal of Fluid Mechanics 646, S. 127, 2010). Folglich lässt sich nicht nur für den Zerfall, sondern auch für die Ausbreitung der Turbulenz eine Halbwertszeit bestimmen. Diese fällt mit sinkender Reynoldszahl sehr schnell ab (rechter Ast in Grafik A.3). Damit war die Lösung des Problems plötzlich ganz einfach. Offenbar überwiegt bei niedrigen Reynoldszahlen der Zerfall der Turbulenz. Dadurch wird die gesamte Strömung irgendwann wieder laminar. Bei hohen Reynoldszahlen hingegen lösen sich 
turbulente 'puffs' langsamer auf, als neue entstehen. Folglich breitet sich die Turbulenz aus und erfasst schließlich die gesamte Strömung. Der kritische Punkt liegt nun genau dort, wo diese beiden Vorgänge sich die Waage halten, also die Kurven der Halbwertszeiten für Ausbreitung und Zerfall sich schneiden. Das ist bei einer Reynoldszahl von 2040 der Fall (Science 333, S. 192, 2011). Von uns durchgeführte Computersimulationen bestätigten dieses Ergebnis. Nach über 100 Jahren konnten wir also eine grundlegende Frage der Strömungslehre mit immenser praktischer Bedeutung endgültig beantworten. Eine besonders wichtige technische Anwendung ist der Transport von Flüssigkeiten und Gasen durch Rohre - etwa Erdöl und Erdgas durch Pipelines. Hier sind Turbulenzen unerwünscht, da sie viel Energie kosten. Ein besseres Verständnis ihrer Entstehung könnte Wege aufzeigen, sie mit geringem Energieaufwand im Keim zu ersticken. Allerdings erfordert das noch tiefere Einblicke in den Mechanismus, wie Wirbelflecken sich aufspalten. Daran arbeiten wir am Max-Planck-Institut für Dynamik und Selbstorganisation in Göttingen nunmehr mit Nachdruck. Mathematisch handelt es sich beim Umschlag von laminarer zu turbulenter Strömung übrigens um einen so genannten Nichtgleichgewichtsphasenübergang, der den gleichen Gesetzmäßigkeiten gehorcht wie die Ausbreitung von Waldbränden oder Epidemien.

\section{Die Autoren}

Kerstin Avila fertigt am Max-Planck-Institut für Dynamik und Selbstorganisation in Göttingen ihre Doktorarbeit über das Thema dieses Beitrags an. Björn Hof, ihr Betreuer, leitet dort als promovierter Physiker eine Arbeitsgruppe, die über die Entstehung von Komplexität und Unordnung in Strömungen forscht. 